Florida International University FIU Digital Commons

$11-17-2014$

\title{
Santeria as an Informal Psychosocial Support Among Latinas Living with Cancer
}

Adelaida M. Rosario

Florida International University, arosa018@fiu.edu

DOI: $10.25148 /$ etd.FI14110744

Follow this and additional works at: https://digitalcommons.fiu.edu/etd

Part of the Social Welfare Commons, and the Social Work Commons

\section{Recommended Citation}

Rosario, Adelaida M., "Santeria as an Informal Psychosocial Support Among Latinas Living with Cancer" (2014). FIU Electronic Theses and Dissertations. 1643.

https://digitalcommons.fiu.edu/etd/1643 


\section{FLORIDA INTERNATIONAL UNIVERSITY}

Miami, Florida

\section{SANTERÍA AS AN INFORMAL PSYCHOSOCIAL SUPPORT AMONG LATINAS LIVING WITH CANCER}

A dissertation submitted in partial fulfillment of

the requirements for the degree of

DOCTOR OF PHILOSOPHY

in

SOCIAL WELFARE

by

Adelaida M. Rosario 
To: Dean Michele Ciccazzo

R.Stempel College of Public Health and Social Work

This dissertation, written by Adelaida M. Rosario, and entitled Santería as an Informal Psychosocial Support Among Latinas Living with Cancer, having been approved in respect to style and intellectual content, is referred to you for judgment.

We have read this dissertation and recommend that it be approved.

$\begin{array}{r}\hline \text { Richard L. Beaulaurier } \\ \hline \text { Paul H. Stuart } \\ \hline \text { Albert Wuaku } \\ \hline \text { Mario de la Rosa, Major Professor }\end{array}$

Date of Defense: November 17, 2014

The dissertation of Adelaida M. Rosario is approved.

Dean Michele Ciccazzo R.Stempel College of Public Health and Social Work

Dean Lakshmi N. Reddi University Graduate School

Florida International University, 2014 


\section{DEDICATION}

This is dedicated to the next generation of Rosario’s: Zoey, Carolina, and the little ones who have yet to join us. May this accomplishment of mine pale in comparison to the wondrous things you will achieve and contribute to the world. 


\section{ACKNOWLEDGMENTS}

A single tree does not make a forest Ifá parable

It would be impossible to attribute this accomplishment to my own merit or efforts. I share this success whole heartedly with the forest of which I am a part and which has made this possible for me to achieve. To my Grandparents, Abuelo and Abuela (Romelio \& Felipa), I will always push as hard and far as I can to make the best of the treasure you sacrificed everything for...opportunity. Know that your struggles were not in vain. Thank you for the better life you have given us. To my parents, Jorge \& Maria, who have offered endless support and unconditional love. Thank you for your patience, for giving me space to shape my own identity, and for always cheering me on.

To my family who are friends: Tia (Adelaida), Jorge Jr., Monica, Javier, Stephanie A., Terri, Juan, Stacy, Stephanie, and the Estevez’s, thank you for being my most amazing and enjoyable foundation. Time spent with you is always time well spent.

To my friends who are family: Florie, Jackie, Marius, Francys, Clara, Beverly, Gaby, Nitza, Aidith \& David, Marisabel \& Carlos, Greg \& Debbie, Claudio, Andy, Gira \& Ana Maria, Julieta \& Tito, Sheila \& Mike, Espy \& Lazaro, and Isabel, thank you for all of your heartfelt encouragement, genuineness, and countless moments of pointless laughter, comfortable quiet, and the best conversations ever to be had.

To my advisor, Mario, thank you for believing in me. You noticed qualities in me that I had not yet discovered about myself and persisted with developing them. Your wisdom and patience will forever be appreciated.

To my committee members, Richard Beaulaurier, Paul Stuart, and Albert Wuaku, thank you for your time and guidance throughout this experience and process. 
To my new colleagues at NIMHD who have quickly become friends, thank you for cheering me on during the last leg of this race and for your generous welcome.

To dear Mariana who serves quite a multi-faceted role in my life as friend, mentor, regulator, and confidant. You are a true ally and us two are a lucky bunch indeed. I am truly fortunate and thankful to have had you by my side through all of this.

To my Godparents who help me navigate the blueprint of life. Thank you for never giving up on me, for opening not only doors but my eyes to growth and endless opportunities, and for providing the tools and spiritual guidance that made this possible.

To all of the unforeseen forces in my life that are my anchors and guiding light. Thank you for your company and blessings as I follow the path you pave before me. And finally to the greatest teacher, my daughter Zoey. How can I possibly put into words what you mean to me? Thank you for inspiring a clarity and drive I've never known before. I will always strive to give you the world since I now know no other world but you.

This group of individuals forms the nurturing foundation that has kept me grounded. The roots extend deep allowing me to stretch upward and grow in ways I never dreamed possible. They were the burning engines that sustained my progress and, during the most difficult of times, the thrusters that forced me forward against the current.

This signals the end of a chapter in my life. The journey is well under way with so much more ground to cover. I take the next steps overwhelmed with feelings of humility and gratitude, and the joy of doing so with the camaraderie of those that comprise this amazing, thriving forest of which I am a part. I love and thank you all. 


\section{ABSTRACT OF THE DISSERTATION}

\section{SANTERÍA AS AN INFORMAL PSYCHOSOCIAL SUPPORT AMONG LATINAS \\ LIVING WITH CANCER}

by

Adelaida M. Rosario

Florida International University, 2014

Miami, Florida

\section{Professor Mario de la Rosa, Major Professor}

Among Latinos, Santería functions as both a religion and a health care system in occurrences of health versus illness within various Latino sub-groups in the U.S. This exploratory study offers a comprehensive analysis of the function of the folk healing tradition Santería as a culturally congruent informal mental health support that assists with coping with the psychosocial sequelae of living with cancer among Latinas in Miami-Dade County, FL. It (a) determined the attitudes of Latinas living with cancer towards Santería as an informal mental health support and (b) explored how Santería offers Latinas effective mental health support that assists in coping with the psychosocial sequelae of living with cancer. The mechanisms and characteristics underlying the motivations of Latinas living with cancer to seek and integrate this informal modality for their cancer care were identified. A purposive sample of 15 Latinas ages 18 and older in Miami-Dade County who had received a diagnosis of cancer were recruited from sites in Miami-Dade offering formal mental health support services and botánicas. Data collection incorporated in-depth interviews and a validation focus group. In an effort to generate theory through a modified Grounded Theory approach, data analysis was 
accomplished by means of multiple coding passes and the constant comparison method which resulted in higher levels codes that were grouped into three major themes: 1) Participants’ Experience with Folk Healers, 2) Influence of Santería on the Cancer Experience, and 3) Participants' Experience with Conventional Healthcare and Mental Healthcare. Results illustrate how, among Latinas, the folk healing tradition of Santería co-occurs with professional medical and mental health treatment in what Arthur Kleinman defines as the popular sector, which identifies and sets the parameters for culturally acceptable forms of healthcare and mental health treatment options. 


\section{TABLE OF CONTENTS}

CHAPTER

PAGE

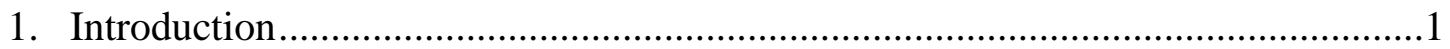

Purpose of Research..................................................................... 7

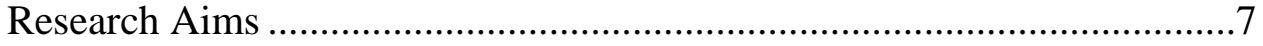

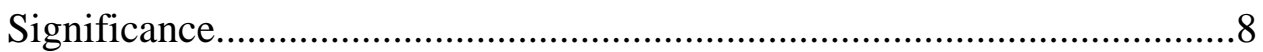

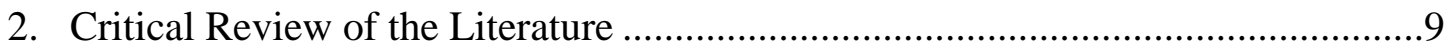

Psychosocial Sequelae of Living with Cancer...........................................9

Mental Health Service Utilization by Latinas...........................................13

Formal Mental Health Services \& Cultural Congruence ...........................15

Latino Culture and Religion \& Spirituality .............................................19

Latinos’ Use of Folk Healing \& Traditional Medicine.............................21

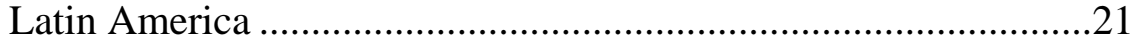

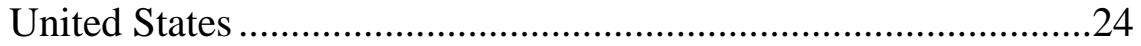

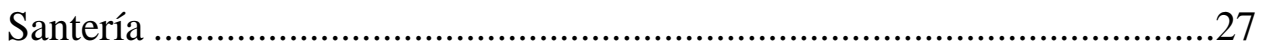

Santería in Cuba .....................................................................29

Santería in the Americas and the United States ............................32

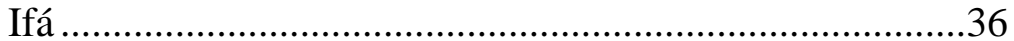

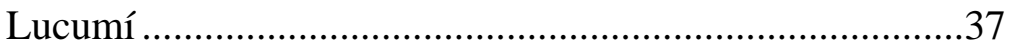

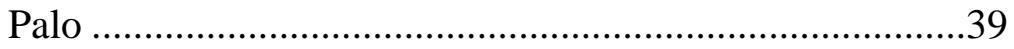

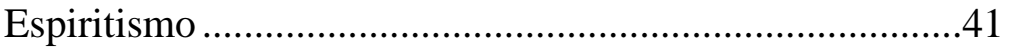

Santería \& Latino Mental Health Care ....................................................43

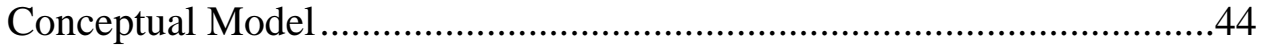

Research Objectives.........................................................................47

Research Questions ...........................................................48

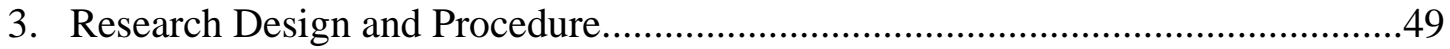

Qualitative Exploratory Research Strategy ..........................................49

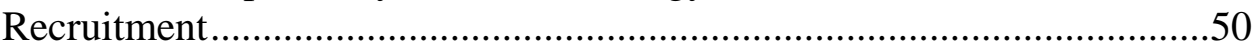

Community-Based Organizations ..............................................50

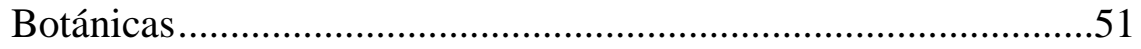

Stage One Data Collection: In-depth Interview .......................................52

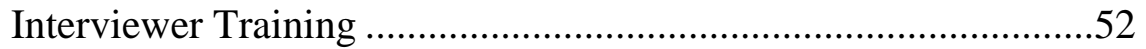

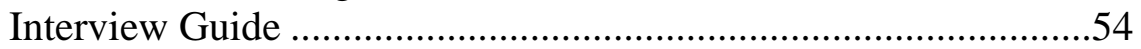

Transcription of Audio Recordings ..........................................54

Stage One Data Analysis .......................................................................55

Computer Assisted Qualitative Data Analysis Software

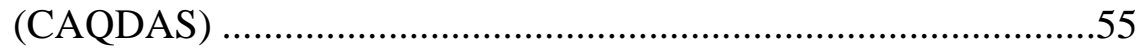

Codes \& the Coding Process..........................................................55

Constant Comparison Analysis..................................................56

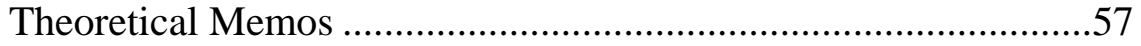

Theory Development ...............................................................58 
Stage Two Data Collection: Focus Groups.................................................58

Member Checking.........................................................................58

Training of Focus Group Facilitator ............................................59

Sample Selection \& Protocol ........................................................60

Stage Two Measurement \& Analysis ..........................................................61

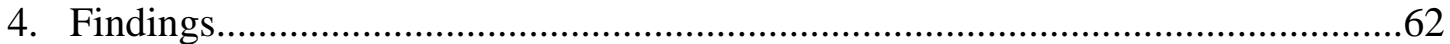

Sample \& Eligibility Criteria ....................................................................62

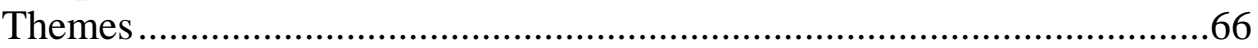

Participants’ Experience with Folk Healing ...................................67

Trust in Folk Healers .......................................................68

Personal Relationship with Folk Healers ............................69

Vested .............................................................................70

The Experience of Santería.................................................71

The Spiritual Entities of Santería .........................................72

Tangibility of Santería Folk Healing ...................................72

Doubt........................................................................73

Influence of Santería on the Cancer Experience..............................74

Metaphysical Influence........................................................74

The Spiritual Component........................................................75

The Cancer Experience ........................................................76

Diagnosis................................................................76

Treatment .................................................................78

Social Role ..........................................................79

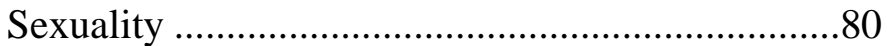

Importance of Psychosocial Health during the Cancer

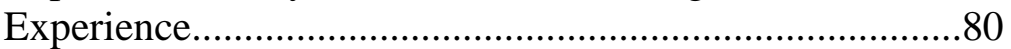

Psychosocial Effect of Santería Folk Healing on the

Cancer Experience ................................................................82

Cured of Cancer ..................................................................85

Participants' Experience with Conventional Healthcare and

Mental Healthcare ..........................................................................86

Comparison to Medical Doctors ..........................................87

Comparison to Formal Mental Healthcare............................89

Therapists (Social Workers, Psychologists,

Psychiatrists) - Experience ........................................89

Therapists (Social Workers, Psychologists,

Psychiatrists) - Perceptions ....................................91

Support Groups - Experience ..................................91

Support Groups - Perceptions ................................92

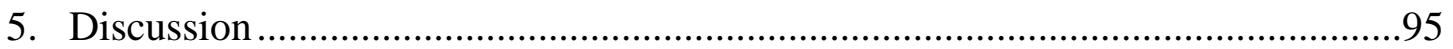

Specific Aims \& Research Questions ....................................................96

Focus Group .......................................................................................99

Interview with Oba Ernesto Pichardo ........................................................102 
Social Work Practice Implications......................................................103

Micro Level - Service Delivery ...................................................103

Meso Level - The Community ...................................................106

Macro Level - Policy ............................................................106

Study Limitations....................................................................107

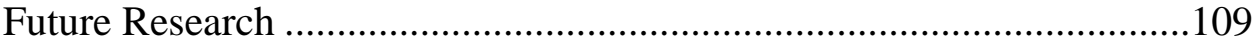

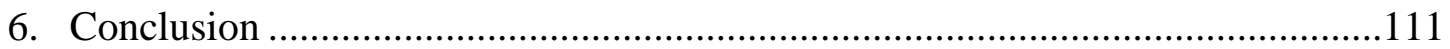

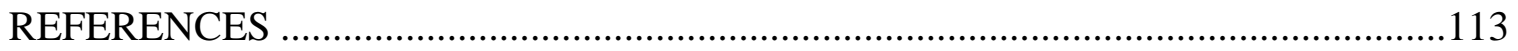

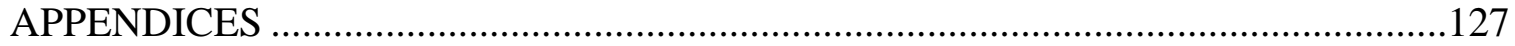

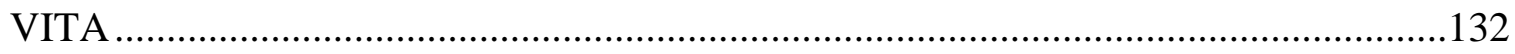




\section{CHAPTER 1}

Introduction

The Institute of Medicine (IOM) reports the psychosocial impact of living with cancer is under-studied and an exacerbated problem for Latinos as well as other racial and ethnic minorities (2008). Cancer is the second most common cause of death in the United States and was recently determined to be the leading cause of death (22\%) among Latinos. Current prevalence estimates indicate that over 13 million people in the United States are living with cancer and about 1,665,540 new cases are expected to be diagnosed in 2014 with 810,320 estimated to be among women nationally, and 114,560 of new cases (among men and women) are estimated to occur in Florida (American Cancer Society, 2014). The American Cancer Society estimates there were about 53,600 new cancer cases in men and 59,200 cases in women diagnosed among Latinos in 2012, with about 17,400 Latinos and 15,800 Latinas estimated to have died from cancer the same year (2014). As the largest and fastest growing minority group in the U.S. numbering 50.5 million, or $16 \%$ of the total U.S. population in 2010, the cohort of Latinas living with cancer and its psychosocial sequelae will grow as well (Humes, Jones, \& Ramirez, 2011; Napoles-Springer, Ortiz, O’Brien, \& Diaz-Mendez, 2009). In Florida, the Latino population made up roughly $23.2 \%$ of the total state population in 2012 , with 103,783 new cancer cases reported among Latinos in 2009 and 49,249 of those cases having been among Latina women (Florida Cancer Data System, FCDS, 2014). In Miami-Dade County specifically, where $64.3 \%$ of the population was reported as Latino in 2012, cancer is the second most common cause of death with 11,462 new cases reported in 2009 and responsible for 3,984 of deaths the same year (Florida Cancer Data System, 
FCDS, 2014; Office of Minority Health, U.S. Department of Health and Human Services, 2014).

Cancer disparities have become part of the larger public health dialogue about health equity in the United States due to these persistent inequities experienced by Latinos and other minority ethnic groups (Meade, Menard, Luque, Martinez-Tyson, Gwede, 2011). As further explained on page 17 of this paper, Latina women living with cancer face unique cultural and socioeconomic factors which pose an increased risk of psychosocial sequelae (i.e., depression, anxiety, shock, disbelief, uncertainty, fear, relational difficulties, decreased health related quality of life, concern over lack of social support, etc.) (Napoles-Springer et al., 2009). In comparison to their white non-Latina counterparts, Latinas experience greater concern over recurrence, pain, death, complications of adjuvant therapy, body image, sexual functioning, job disruptions, financial hardships, weight gain, and being rejected by their husbands after treatment (Napoles-Springer et al., 2009). In addition, “limited English proficiency, part-time or no employment, limited insurance coverage, lack of transportation, and unfamiliarity with the health care system may elevate their risk of distress” (Napoles-Springer et al., 2009, p. 268). Moreover, Moyer Knapp-Oliver, Sohl, Schnieder, \& Floyd (2009) suggest that “most cancer patients do not make use of psychosocial resources naturalistically” (p. 346) and instead require assistance and encouragement in seeking and utilizing mental health services for the psychosocial sequelae. For Latinas, the increase in psychosocial sequelae combined with a general proclivity of neglecting to seek formal psychosocial treatment results in a pressing need for culturally congruent psycosocial care that resonates with Latinas. Latinas, however, are more unlikely than other demographic groups to access 
culturally and linguistically appropriate formal mental health services (i.e., support groups, counseling, etc.) for their psychosocial sequelae needs (Ashing-Giwa, 2008; Galvan, Buki, \& Garces, 2009; Comas-Diaz, 2013; Napoles-Springer et al., 2009). An investigation by Buki and colleagues (2008) of community based organizations serving Latina oncology patients suggests an inadequacy in support groups and mental health practitioners available to provide culturally congruent care for this population. Furthermore, Buki et al. (2008) indicated that Latinas with female cancers (breast and gynecological cancers) who met criteria for major depressive disorder were "grossly underserved," with less than $4 \%$ participating in support groups or meeting with a mental health professional, and only 12\% reporting taking medication (p. 163). Lack of access to treatment can be attributed to low income, lack of health insurance, limited English proficiency and other systemic barriers (Martinez, Aguado, Martinez, Flores, \& Meade, 2008).

The overall lack of access to quality, culturally congruent mental health services is not confined to Latinas with cancer, but to the Latino community in general as well, resulting in their routine use of alternative forms of care. Among Latinos, indigenous or folk healing traditions such as Santería have gained recognition for their poly-functional role not only as a religion, but as an informal health and mental health care system (Pasquali, 1994). Santería offers Latinos a collective, culturally relevant perspective on health, illness, and appropriate treatment options.

A collective worldview internalizing the belief that one's spirit must be attended to for effective and treatment is popular among various Latino groups (Ortiz, Davis, \& McNeill, 2008). Illness and health are believed to be strongly influenced by spiritual and 
religious factors, which is a fundamental tenet of Santería. Santería is an amalgamation of popular Catholicism, 19th century French Kardecian spiritism, native pre-colonial Cuban Indian beliefs and practices, and a variety of African traditions that emerged as a result of Cuba’s socio-cultural and political colonial history (Brandon, 1997). Santería spread throughout the Caribbean and into other Latin American countries where there was already a presence of and familiarity with African Diasporan traditions (Murphy, 1994). Consequently, the various Latino subgroups in the U.S. each possess a unique native religious heritage with similarities to Santería that Murphy (1994) states can partially be explained to their historical link to Africa. In addition to Curanderismo and Espiritismo, Santería is identified by Gloria and Peregoy (1996) as a main healing system utilized by various Latino subgroups and communities in the U.S. Sandoval (2008) described Santería as the single most visible informal treatment modality in present day MiamiDade County by Sandoval (2008).

Despite a 2010 report based on a national public opinion survey by the Pew Hispanic Center (2010) indicating that less than $10 \%$ of the Latino population uses a folk/traditional treatment, there is growing evidence that Latinos commonly utilize traditional healers practicing Santería as a main resource in attending to pressing health and mental health needs in lieu of, or alongside, formal services as reported by Viladrich who ethnographically examined botánicas and interviewed folk healers (2006). In 2007, "traditional healer" was included as one of 36 complementary and alternative medicine (CAM) modalities in the National Health Interview Survey (NHIS) conducted by the Centers for Disease Control and Prevention's (CDC) National Center for Health Statistics (NCHS) (Barnes, Bloom, \& Nahin, 2008). Hsiao and colleagues (2006) reported CAM 
usage variation between minority ethnic groups in a cross-sectional study conducted in California and reported that an ethnic group is more likely to use types of CAM that are consistent with their cultural foundation, defining "ethnic-specific" CAM use as "using a type of CAM that is particularly congruent with a racial/ethnic group's cultural identity” (p. 282). Studies conducted by Insaf, Jurkowski, and Alomar (2010), Olsen (2009), and Villa-Caballero et al. (2010) also indicate CAM use among minorities is closely associated with their ethnic background, identity, and with the values, beliefs, spirituality, worldview, and philosophical orientations of life and health.

In a study by Schoenberg, Stoller, Kart, Perzynski, and Chapleski (2004) focused on older diabetes patients, results indicated Latinos had a higher CAM use (40\%) than African Americans (20\%), Native Americans 15\%, and Caucasians (15\%). Shoenberg and associates (2004) also found that among all ethnic groups sampled, Latinos chose CAM modalities and practitioners who were congruent with their cultural background (Schoenberg et al., 2004). Among Latinas, Laws and Carballeira (2003) demonstrated in a study conducted in Massachusetts with women over the age of 40, 59\% of the Latina sample, which was 96\% Puerto Rican or foreign born, used at least one non-conventional treatment in the last year (Insaf et al., 2010).

These CAM modalities utilized by Latinos offer ethnic and cultural congruency since many originate in their indigenous healing traditions and are infused with a religious and spiritual component which is foundational in Latino culture (Insaf et al., 2010; Reyes-Ortiz, Rodriguez, \& Markides, 2009). Hsiao and colleagues (2006) demonstrated that Latinos have the highest use of Curandero traditional healers in their sample. Suarez, Raffaelli, and O’Leary (1996) and Villa-Caballero et al. (2010) reported 
on Latinos' particular use of traditional healers in managing chronic illness such as HIV and diabetes, respectively, with Ashing-Giwa, Padilla, Bohorquez, Tejero, and Garcia (2006) reporting on the use of traditional healers by Latinas specifically contending with breast cancer.

Also contrary to the 2010 Pew Hispanic Center findings indicating use of folk healers in less than $10 \%$ of Latinos, health researchers such as Viladrich (2006) continue to document the presence of stores known as botánicas that serve as an alternative source for health/mental health care services and products among Latinos (Jones, Polk, FloresPeña, \& Evanchuk, 2001). Botánicas are the principle retail outlet providing familiar sacramental and religious supplies (herbs, plants, and spiritual ingredients) needed to support the practice of Santería, as well as other Latino folk healing traditions (Espiritismo, folk Catholicism, etc.). They are a community-based resource serving a variety of Latino sub-groups thus making it a point of entry into the Latino community and a source of spiritual, family, and personal counseling, and provider of crucial ingredients for various illness remedies (Jones et al., 2001). Botánicas proliferate and are highly visible in Latino saturated communities indicating a demand for supplies. A search for "botánicas" in "Miami, FL" on yellowpages.com conducted by this author on August 2014 listed over 75 shops in the Miami-Dade County area. They are tangible and material proof of the thriving economy of Santería’s presence among a pan-ethnic population of Latinos in urban areas such as Miami (Viladrich, 2006).

Among Latinas, several studies have found that culturally embedded traditions such as Santería continue to thrive as resources and natural informal supports (Sandoval, 2008). Such traditions offer an alternative approach to health/mental health and healing 
despite the increased availability and access to formal, western bio-medically based services (Baez \& Hernandez, 2001; Viladrich, 2006). Yet research delineating the motivation for seeking this "ethnic-specific" form of alternative medicine among Latinas is limited, particularly in identification of the specific characteristics and processes utilized by Santería traditional healers that render such informal treatments effective in mitigating the psychosocial sequelae experienced by Latinas with cancer. Particular attention focused on Latinas' motivation for use of this specific form of culturallyinfluenced folk/traditional system of healing is needed (Hsiao et. al., 2006).

Purpose of Research

This exploratory study serves to address the gap in knowledge concerning the health-seeking behavior of Latinas living with the experience of cancer. There is a lack of research that examines the attitudes and underlying intentions of Latinas with cancer who seek treatment by Santería folk healers. This study examines the impact of Santería on the health seeking behavior of Latinas living with cancer in Miami-Dade County by means of the following two aims.

\section{Research Aims}

Aim 1: Determine the attitudes of Latinas living with cancer towards Santería as a mental health support that assists in coping with the psychosocial sequelae of living with cancer. Aim 2: Explore whether Santería offers Latinas effective mental health support that assists in coping with the psychosocial sequelae of living with cancer and if so, how? 


\section{Significance}

It is estimated that over the next 20 years, cancer cases in the Latino population will increase by $142 \%$ compared to a $42 \%$ increase projected for the total U.S. population based on research conducted by MD Anderson Cancer Center (Justice-Gardiner, Nutt, Rechis, McMillan, \& Warf, 2012). Since the cancer experience is defined by one’s cultural impression of health and illness, there is an urgent need for healthcare services intended for Latinos to be guided by Latino cultural norms and values inclusive of the nuances that guide social interactions (Insaf et al., 2010; Martinez et al., 2008). The findings from this study are expected to enhance our understanding of the importance and relevance of culturally congruent services to Latinas living with cancer. The study seeks to understand how Santería functions as an informal mental health treatment modality from a religious coping perspective. The aims when achieved are intended to gain a specific understanding on Latinas' views on their cancer experience and their psychooncological needs. A clear sense of the motivations behind the health seeking behavior of Latinas living with cancer is needed to inform such services. Thus, the proposed study aims have been achieved by gaining an emic, client-centered notion. The findings of this study contribute to the field of Social Work and practice by furthering knowledge on the meaningful characteristics of effective, quality psycho-oncological care as determined by Latinas to guide the development of culturally-congruent psychosocial interventions and treatments for this population. Most importantly, these findings can contribute to the United States health care system's active attempt to broaden approaches towards health care equity among diverse populations (Gurung, 2013). 


\section{CHAPTER 2}

\section{Critical Review of the Literature}

Psychosocial Sequelae of Living with Cancer

Despite the race or ethnicity of an individual, a cancer diagnosis marks an immediate passage from a condition of health to one characterized not only by physical changes but by uncertainty, the threat of death, and social role disruption (Annunziata, Muzzatti, \& Bidoli, 2011). Multiple emotions are experienced upon receipt of a diagnosis including shock, disbelief and despair, anger, fear, sorrow thus increasing the need for social support (Martinez et al., 2008). Treatment following diagnosis is typically very challenging physically to patients, requiring some combination of surgery and adjuvant therapy such as radiation or chemotherapy for months or years (IOM, 2008). The consequences of psychological and social distress caused by diagnosis and treatment is recognized as a component in the process of disease adjustment and identified as the “psychosocial sequelae” of the cancer experience (Annunziata et al., 2011).

The impact of disease via the diagnosis initiates what is widely viewed as an existential crisis that, in the first three months from diagnosis, arises in $70 \%$ of patients (Annunziata et al., 2011; Spencer et al., 1999). The crisis is characterized as "a multifactorial unpleasant emotional experience of a psychological (cognitive, behavioral, emotional), social, and/or spiritual in nature that may interfere with the ability to cope effectively with cancer, its physical symptoms and its treatment” (IOM, 2008, p. 357). Anunziata and colleagues (2011) define the first three months after receipt of a diagnosis as the "diagnostic phase” characterized by initial shock. The majority of patients develop active coping strategies thereafter during the "therapeutic phase," however, $20-30 \%$ of 
oncology patients continue to live with elevated distress levels in the therapeutic phase several months after diagnosis (Anunziata et al., 2011). Additionally, there has been a measurable increase in symptoms of psychosocial distress due to the complexity of modern day cancer treatments, which have placed a considerable emotional and social burden on patients and their families (Sellick \& Edwardson, 2007).

Not surprisingly, significant mental health problems such as depression and anxiety disorders are common in oncology patients (Spiegel \& Giese-Davis, 2003). Studies by Andrykowski and Kangas (2010), Goncalves, Jayson, and Tarrier (2011), and O’Connor, Christensen, Jensen, Moller, and Zachariae (2011) have also documented the presence of symptoms meeting the criteria for post-traumatic stress disorder (PTSD) and post-traumatic stress symptoms (PTSS) in those with cancer. These mental health problems are additional contributors to functional impairment in carrying out family, work, and other societal roles, poor adherence to medical treatments, and adverse medical outcomes (Katon, 2003).

In a study by Ashing-Giwa and colleagues (2004), it was noted that women who experience breast cancer generally have heightened psychosocial concerns regarding their children, family, body image and sexual health, amount of time spent with providers, the cultural sensitivity of providers, and cultural beliefs about illness, gender role, and in particular, prioritizing family obligations and role as caregiver above one's health. Ashing-Giwa et al. (2004) and Yanez et al. (2011) indicate the impact of cultural influence and socio-ecological contexts on the psychosocial experience of Latinas who experience higher rates of advanced cancer and rigorous treatments face additional culturally-relevant stressors upon a breast cancer diagnosis and which create a greater 
psychological burden. In a multi-ethnic cancer study that included Latinas, Spencer and colleagues found Latinas to have significantly higher concerns about pain, survival and sexuality (1999). In a qualitative study on the cancer experience and quality of life conducted by Fatone, Moadel, Foley, Fleming, \& Jandorf (2007), the primary domain of concern for the Latinas sampled was psychological.

Psychosocial services and interventions have been shown to generally mitigate the negative psychosocial sequelae of the cancer experience and improve the quality of life of patients (Graves, 2003). As delineated by the Institute of Medicine, psychosocial health services are:

psychological and social services and interventions that enable patients, their families, and health care providers to optimize biomedical health care and to manage the psychological/behavioral and social aspects of illness and its consequences so as to promote better health. (2008, pp. 9)

These formal psychosocial oncological services and treatments encompass a range of interventions. They vary based on: 1) therapeutic technique and conceptual framework (i.e., cognitive behavioral, psycho-educational, art, mind-body, massage, etc.), 2) specific cancer site (i.e., breast, lung, prostate, leukemia, etc.), 3) cancer stage (early non-metastatic versus late \& metastatic), 4) population (i.e., patient, patient’s spouse, children of an oncology patient, etc.), 5) point in the "survivorship continuum" (i.e, newly diagnosed, post-surgery, during adjuvant therapy, post-treatment, long term survivorship, etc.), and 6) psychosocial factors (i.e., wellness, quality of life, anxiety, depression, uncertainty, self-efficacy, end of life issues, etc.) (Holland \& Weiss, 2010). By targeting overall aspects of emotional well-being and health related quality of life 
factors, these services and interventions do well in appropriately addressing the psychosocial needs of oncology patients. Unfortunately, the healthcare system, which is rooted in the biomedical model, invests most resources to address the physiological components of the illness, often failing to address the psychosocial sequelae.

Annunziata and colleagues (2011), Sellick and Edwardson (2007), and Hopwood, Sumo, Mills, Haviland, and Bliss (2010) indicate as much as 50\% of oncology patients experience psychological distress, and it has been suggested that less than half of all cancer patients receive adequate mental health intervention for the psychosocial distress experienced. Factors such as: 1) difficulty in oncology or primary care providers considering psychosocial support an integral part of care or their failure to identify and differentiate normal psychological responses from signs/symptoms of psychiatric malaise, 2) difficulty for the patients to express their situation and needs, and 3) the scarce investment in the psychological sector on the part of institutions all create barriers for oncology patients from receiving adequate mental health care for the psychosocial sequelae (Annunziata et al., 2011; IOM, 2008).

Additional barriers to receiving adequate mental health care for psychosocial oncology needs exist for racial and ethnic minorities. Latina oncology patients are less likely than non-minority patients to 1) access and 2) receive quality, culturally and linguistically appropriate formalized psychosocial services, which complicates the identification of their psychosocial needs (Ashing-Giwa, 2008; Galvan et al., 2009; Napoles-Springer et al., 2009) Moreover, cultural beliefs such as 1) reluctance to discuss emotional problems and personal feelings with strangers and 2) viewing psychosocial support as services for the mentally ill have been found to deter ethnic minorities from 
accessing psychosocial care and further prevent the psychosocial needs of Latinas from being met through formal mental health services (Ashing-Giwa et al., 2004; NapolesSpringer et al., 2009).

\section{Mental Health Service Utilization by Latinas}

Despite the research indicating the benefits of mental health services in improving overall psychosocial health for women with cancer, these formal services are not adequately reaching Latinas. Evidence suggests that most oncology patients require assistance and encouragement in seeking and utilizing mental health services for their psychosocial needs (Moyer et al., 2009). The need for this assistance is exacerbated for Latinas whose access and use of mental health services is further limited and influenced by financial, linguistic, institutional, and cultural factors (Galvan et al., 2009).

Latinos delay or refuse to seek needed mental health services as a result of past experiences and expectations of mistreatment due to perceived prejudices and discrimination in overall interactions with the healthcare system and organizations (Smith, Sudore, \& Perez-Stable, 2009). This discrimination is evident in the mental health services Latinos receive which are reportedly poorer in quality resulting in worse outcomes than those experienced by non-Hispanic Whites (Elder, Ayala, Parra-Medina, \& Talavera, 2009). There are also certain cultural characteristics found among Latinos that are "generally forgotten by or are unknown to mental health specialists who have received classical training” (Ruiz \& Langrod, 1976, p. 393). The limited availability of support and negligible quality from formal sources (i.e., mental health professional, support groups, research clinical trials, and community organizations) results in 1) 
Latinas' decreased awareness and access to key information that can promote health adjustment to cancer and 2) Latinas overall underutilization of mental health treatment (Carpenter-Song, Whitley, Lawson, Quimby, \& Drake, 2011; Galvan et al., 2009; Institute of Medicine, 2002).

Much of the current underutilization of mental health services is rooted in a historical institutional discrimination that still pervades the U.S. healthcare system (McBride, 2011). Keefe and Casas (1980) discuss literature dating back to the 1930s, which substantiates concerns regarding the underutilization of mental health services among Mexican Americans. At the time of their investigation in 1980, Keefe and Casas concluded Mexican Americans continued to underutilize public mental health services. In a meta-analysis conducted more recently on Latino adults and use of mental health services by Cabassa, Zayas, and Hansen (2006), their analysis found that Latinos in the United States consistently underutilize mental health services compared to non-Latino Whites, and when attending to mental health concerns, Latinos prefer general or primary care physicians over mental health professionals. Furthermore, in a study by Pescosolido, Gardner, and Lubell (1998), Latinos were found to incorporate different combinations of sources of health care and support which included family members, friends, and spiritual leaders. These findings from Pescosolido and colleagues (1998) suggest that factors beyond socioeconomic access barriers contribute to the underutilization of formal mental health care (Lopez, 2002). This is unsurprising given that Cheung and Snowden (1990) explain mental health exists as a culturally embedded notion where 1) beliefs about the nature of mental health problems are rooted in folk criteria, 2) expectations of the 
healthcare environment and provider/patient relationship are created, and 3) the acceptability of alternative solutions affect the utilization of formal services.

\section{Formal Mental Health Services \& Cultural Congruence}

The underutilization of formal mental health services for the psychosocial sequelae needs of Latinas with cancer is well documented, as well as other race/ethnicitybased inequities in cancer care (Jean-Pierre et al., 2010). Studies consistently find that lower rates of health insurance coverage contribute to the disparity in quality and access to care for the Latino population in the U.S. (Carpenter-Song et al., 2011). Perez, Ang, and Vega (2009) estimate that as much as a third of the Latino population in the U.S. lacks health insurance and Ritter and Hoffman (2011) indicate that Latinos have the highest uninsured rates of any racial or ethnic group in the U.S. Livingston of The Pew Hispanic Center (2009) reports that 60\% of undocumented and 28\% of Latino citizens or permanent residents are uninsured (compared to 15\% of non-Hispanic Whites, 22\% of non-Hispanic Blacks, 34\% of American Indians/Alaska Natives, 14\% of Asians/Pacific Islanders, and 17\% of the adult U.S. population) (Moonesinghe, Zhu, \& Truman, 2011). Aside from access issues attributed to lack of health insurance, inequities that exist in the U.S. healthcare system (inclusive of mental health care) are also precipitated by the cultural incongruence of services afforded to racial/ethnic minorities. Healthcare disparities are defined by the Institute of Medicine (2002) as "racial or ethnic differences in the quality of health care that are not due to access-related factors or clinical needs, preferences, and appropriateness of intervention” (p. 32). IOM's description of care disparities supports the notion of cultural congruence as an essential component in 
creating effective services for Latinos and other racial/ethnic minority groups (NapolesSpringer et al., 2009). Implementation of culturally congruent services within the current health system emerged from public health efforts to make healthcare services (inclusive of mental health) more responsive to ethnic minority and underserved populations (Zambrana, Molnar, Munoz, \& Lopez, 2004).

Cultural competence is defined as a "set of congruent behaviors, attitudes, and policies that come together in a system, agency or profession that enables that system, agency or profession to work effectively in cross-cultural situations” (Zambrana et al., 2004, pp. SP37). The term did not begin to appear consistently in the healthcare and mental healthcare literature until the early 1990s (Saha, Beach, \& Cooper, 2008). By May 2007, more than 1000 articles mentioning the terms “cultural competence” or "cultural competency" in their titles or abstracts had been published in medical and nursing journals with more than three-quarters of them appearing since 2000 (Saha et al., 2008). The term's roots are in the early formulations of cross-cultural healthcare proposed by those in the field of medical anthropology, including Arthur Kleinman (1980). These formulations were originally identified as "cultural sensitivity" and "cultural appropriateness" but have evolved into the current term in the academic literature, “cultural congruence.”

Demands for culturally congruent healthcare services, inclusive of mental health, for minorities grew out of the failure of service delivery systems to be responsive to all segments of the population (Chin, 2000). Erwin et al. (2010) discuss a culturally congruent intervention as reflecting "shared perspectives, beliefs, practices, life experiences, and the history of the subgroup to be addressed” (pp. 694). In a systematic 
review of cultural congruence in mental health completed by Bhui, Warfa, Edonya, McKenzie, and Bhugra (2007), only nine out of 109 potential papers included an evaluation of a model to improve cultural congruency practice and service delivery. As immigration and diversity increases, healthcare and mental health service providers and policy makers have realized the profound effects of cultural differences on the patientprovider relationship. Only in more recent times have they proceeded with a more macro perspective giving attention to whether the organizational systems of care in which they function are culturally congruent.

In keeping with the definition of cultural congruence, consideration to a culture's built-in system of beliefs, perceptions, and ideas about health and mental health and illness is needed (Helman, 1994; Kleinman, 1980). Research has shown that culture and ethnic factors influence how one understands, explains, and responds to the fact and meaning of life-threatening diseases such as cancer; how side effects will be understood and experienced; how emotions will be expressed; and which treatments will be sought (Kawaga-Singer, 1995). Additionally, economic, social, spiritual, and family situations contribute not only to the interpretation of the illness but to treatment options as well.

Latino cultural traditions emphasize the importance of collectivism, interdependence, and cooperation (Changrani et al., 2008). These cultural values, which assert powerful influences over Latinas, can also increase their risk for experiencing compounded and heightened levels of distress when living with cancer. Specifically, 1) familismo (the value of the family affecting a Latina's attempt at protecting her family from the consequences of her illness and inability to carry out household responsibilities), 2) personalismo (preference for relating on a personal level rather than at a formal or 
institutional level), 3) marianismo (traditional cultural prescriptive assigned to women rooted in Roman Catholicism), 4) maintaining closer distance and more liberal physical touch during conversation, and 5) faith being the cornerstone of Latino life in many communities, limit the effectiveness of formal mental health care in meeting the psychosocial needs of Latinas with cancer (Changrani et al., 2008). The ethnocentric tradition of Western "professional” psychology and mental health services stress the desirability of individualism, autonomy, and competition which stands at odds with Latino cultural values (Changrani et al., 2008). Such service delivery has sociohistorically viewed Latino cultural values as "pathogenic and in need of reform” (De La Cancela \& Martinez, 1983, p. 252). The cultural experience of Latinos' illness and treatment needs is completely devalued and treatment options lack the incorporation of such foundational cultural values.

Even though formal mental health services for Latinas with cancer do appear to positively influence their social support and survivorship needs, few are receiving such care in an effective manner. Miano, Rojas, and Trujillo (1996) reported that the traditional support group model has not been successful among Latinos (Galvan et al., 2009). However, more culturally congruent interventions have proven effective. Martinez and colleagues (2008) developed a cancer-camp promoting peer support specifically for Latinas in Central Florida. The camp was designed with language and cultural values in mind and was successful in providing culturally and linguistically relevant support to Latinas with cancer (Martinez et al., 2008). In a study conducted by Alferi, Antoni, Ironson, Kilbourn, and Carver (2001) investigating women with early-stage breast cancer, more than one informal treatment option, which included spiritual healing in 
addition to extended family and friends, was shown to be effective and commonly used by most of the Latinas sampled. Results from a study by Changrani and colleagues (2008) also determined that informal support networks, such as extended families and civic clubs, were more helpful and effective for Latinas as compared with non-Hispanic Whites.

Many of these informal options have been endorsed as "complementary and alternative medicine” or CAM, by the dominant, professional convention medical tradition (more recently such treatments are deemed “integrative medicine”). Studies by Applewhite (1995) and Reyes-Ortiz and colleagues (2009) state reasons why patients use these CAM practices include not only barriers to conventional care, but dissatisfaction with conventional care, having desire for control over one's health, having a more holistic view on health, cultural beliefs, and factors related to the physician/patient relationship. Therefore, to find culturally congruent mental health services that effectively incorporate Latino worldview and cultural variables (such as language, norms, and religion), Latinas commonly turn to accepted, popular informal practices found in their community which include folk/traditional healing in addition to family and friends (Gonzalez, 2002). Folk/traditional healing includes a religious/spiritual component which is a core Latino cultural value (Reyes-Ortiz et al., 2009).

\section{Latino Culture and Religion \& Spirituality}

Religion/spirituality is identified as a foundational component of Latinos' reality and culture in both Latin American countries and in the United States. Sanchez (2012) reports that religion plays a vital role in Latin American culture and is a guiding resource 
when attending to life's circumstances. In regards to the cancer experience, Nairn and Merluzzi (2003) found religious coping to be just as important as social support in enhancing self-efficacy which leads to better adjustment of the illness experience. For Latinas specifically, there is a propensity to embrace overt religiosity and spirituality as a result of the Latino cultural values of familismo and marianismo. Ashing-Giwa and colleagues' sample of women, which included Latinas, with breast cancer spoke of their firm spiritual beliefs and practices as being central to their coping and in placing more importance on their spirituality than on health care providers (2004).

The literature on Latinos' religiosity and spirituality identifies a distinction between affiliation to mainstream religious institutions, such as the Catholic Church and Protestant traditions, and use of a folk/traditional system (Pew Research Center, 2007). There is the implication of a customary dual adherence to a mainstream religious institution and a folk belief system among Latinos, yet there is no examination of how this common duality exists without experiencing cognitive dissonance.

Contemporary Latino identity and culture is intimately connected to its religious and spiritual history. The Spanish colonial conquest of Latin America left an irrefutably transformative and lasting impression on the religiosity of Latinos. It historically marks the reshaping of Latinos' religious beliefs and practice into that of mainstream faiths. However, the primordial, ancient spiritual roots of Latinos withstood the colonial indoctrination and instead remain subtly embedded in Latino religiosity and spirituality. This ancient spiritual awareness can be considered what Alan Harwood describes as an 
“ideological ethnicity"1 among Latinos which manifests itself in this persistent observation of folk/traditional belief systems and its application as a healing system (1981). This acceptance of a distinctly Latino ideological ethnicity thus facilitates the successful negotiation between formal mainstream religious affiliation and use of folk/traditional practices (Comas-Diaz, 2006). This interlacing realm of mainstream religion and folk/traditional healing practices seems to be the psycho-spiritual space where religious coping among Latinos takes place. "Religion” for Latinos is inclusive of mainstream faiths and folk/traditional beliefs offering a comprehensive cosmological understanding of what is divine, metaphysical, and sacred. Furthermore, it provides the blueprint on how the metaphysical relates to health, illness, and treatment options.

\section{Latinos’ Use of Folk Healing \& Traditional Medicine}

\section{Latin America}

In all civilizations, indigenous and traditional healers have existed and worked within the realm of physical, psychological, and spiritual medicine (McNeill \& Cervantes, 2008). A study commissioned by the United Nations Development Program stated that approximately $80 \%$ of the world's population relies on traditional healing as an integral aspect of health and illness care (Belliard \& Ramirez-Johnson, 2005; Zapata \& Shippee-Rice, 1999). Hsiao et al. (2006) describes how the many ethnic groups residing in the United States have a rich history of such traditional medicine and Zapata and Shippee-Rice (1999) indicate that Latinos in particular have a long history of aggressively utilizing the traditional healers in their country of origin. Traditional healers

\footnotetext{
${ }^{1}$ A tendency to find meaning in life by revisiting cultural beliefs and rituals
} 
continue to be used by Latinos having acquired their "credibility and validity after having been used for generations” (Zapata \& Shippee-Rice, 1999, p.141).

Most popular, and identifiable, when referring to Latin American folk or traditional healers is the curandero which appeared in the 16th century as a result of the Spanish conquest. The Nahua tícitl, the Huastec ilalix, the Tzeltal h’ilojel and Tzotil h'ilol, the Mayan h-men, and the Pokoman ah cut were "indigenous medical specialists that gradually became a homogenized group generally labeled as curanderos/as who "solved the health problems of their peoples through sacred, ritualistic, and magical practices” (Ortiz et al., 2008, p. 272). These pre-colonial Mayan and Aztec indigenous systems viewed human beings prior to colonization "in an integral and interactive relation with the cosmos, which includes both nature and society” (Garcia, Sierra, \& Balam, 1999, p. xxxiv). The living world was interconnected comprised of the heavens, the metaphysical, the physical, mankind, etc. This tenet holds that all of life's happenings, inclusive of health and mental health versus illness, are a result of this interconnectedness among all living things both material and transcendental. Distinction between health and mental health did not exist independently given that the common cause of illness was judged to be the same: spiritual imbalance. Among the Aztecs, one of the highest ranking healers, tonalpouohqui, was called upon to help the most emotionally and psychologically disturbed individuals by restoring spiritual balance and equilibrium (McNeill \& Cervantes, 2008).

One of the most common indigenous systems found in the Caribbean and within the Americas is the Yoruba-based system of Ifá which promotes spiritual balance and “manages health and mental health concerns via holistic approaches addressing mind- 
body-spirit connections, in addition to kinship bonds that extend beyond biological reactions” (Ojelade, McCray, Ashby, \& Myers, 2011, p. 407). Upon arrival to new lands, Ifá fused with local belief systems and as a result numerous distinct, yet comparable diasporic traditions emerged, of which Santería is the most well-known. Work by Reiff, O’Connor, Kronenberg, Balick, and Lohr (2003) supports Ojelade and colleagues' (2011) discourse on these African Diaspora traditions. They interviewed modern-day Dominican healers from the Caribbean who describe a concern for the emotional and spiritual aspects of illness, perceiving a connection between the natural and supernatural dimensions. These "Latino" traditional healing systems vary but conceptually they focus on spiritual balance and harmony, which affects not only one's spiritual, but physical and mental states (Balick et al., 2000).

In sum, Latin American indigenous and traditional belief systems serve both healing and spiritual functions through the understanding that "the life system extends into a metaphysical belief system in which the worldly and other worldly operate conjointly on a daily basis” (Gloria \& Peregoy, 1996, p. 123). Subsequently, there is value placed on the "whole" person, and the holistic orientation afforded by traditional Latino healing systems is a quality sought when mitigating illness (Zapata \& ShippeeRice, 1999). In both the Latin America and the United States, traditional healers are typically known individuals in the community who share this concern with their clients along with similar experiences, geographic location, socioeconomic status, class, language, religion, and beliefs regarding the causes of pathology (McNeill \& Cervantes, 2008). 


\section{United States}

In the United States, Latinos’ views about health and illness management tend to diverge from the Western biomedical model and tend to continue incorporating traditional beliefs and the use of such healers (Gallant, Spitze, \& Grove, 2010; VillaCaballero et al., 2010). Traditional healers are recognized as possessing a divine gift, or “don”, for healing and deliver a culturally appropriate method of treatment in line with U.S. Latinos’ faith and belief system (Applewhite, 1995). The traditional healer’s office is "the community" and often, the healer's home creating an atmosphere where help can be sought absent of rigid appointments, forms with professional medical jargon, hurried physician and staff, and unmanageable costs (Gurung, 2013). In regards to mental health, traditional healers in the U.S. function in a manner similar to that of health psychologists focusing on social, psychological, and biological issues but treat a spiritual component of the patient as well. Latinos with chronic conditions such as cancer, arthritis, asthma, hypertension, and diabetes face a greater perceived need and are more likely to use this ethnic-specific alternative treatment option believing it helps their overall health (Hsiao et al., 2006; Villa-Caballero et al., 2010).

The presence of chronic and debilitating illness has been consistently found to be a reason people are attracted to and seek out alternative treatment options congruent with their values, culture, and worldview (Chang, Wallis, \& Tiralongo, 2007). However, in the U.S. where the health care providers are educated in conventional medicine, traditional practices of other cultures are discounted. "Folk” traditions are found throughout modern society and influence practically all patients and doctors to some degree (Hufford, 1997). It is a central feature of the cultural differences among patients, yet is also a basic aspect 
of health behavior that all patients have in common (Hufford, 1997). As traditional healers fall under the rubric of "folk" healing or medicine, it is critical to understand the spectrum of definitions used to describe folk medicine since it impacts profusely the perception of the term and such practices used particularly among ethnic minorities in the U.S.

In regards to medical practices, "folk" refers to cultural and societal health systems that are not related to or included in the dominant Western biomedical conventional tradition. William George Black was the first person to use the term "folk medicine" in print in 1878 and defined it as "meant to comprehend the subjects of charms, incantations, and those habits relating to the preservation of health, or the cure of disease, which were and are practiced by the more superstitious and old fashioned" (Hatfield, 2004, xvii). Based on Black’s description of folk medicine, it is obvious the concept absorbed a sense of social and educational inferiority and irrationality since its inception. A related term, "indigenous healing" is defined as "those beliefs, traditions, and strategies that originate within a culture or society and are designated for treating members of a given cultural group” (McNeill \& Cervantes, 2008, p. 3). This emphasis on an "other" cultural origin but more so, the persistent lack of inclusion in the dominant professional medical culture, amplifies "folk" or traditional medicine’s position as a subordinate health system.

The view of folk medicine's inferiority is intimately associated with socioeconomic status and demographic characteristics of those employing folk medical practices. Since its most commonly noted use is among ethnic minorities, folk medical practices lends itself to further correlation with disadvantaged populations, i.e., those 
lacking resources, education, and regarded of low socioeconomic status. Reyes-Ortiz and colleagues (2009) found that curanderos were consulted mostly by foreign-born females of Cuban origin in their sample who reported fair/poor health rather than by U.S. born persons of Central or South American origin with good, very good, or excellent health. In recent work by Gurung (2013), however, curandero use was found among highlyassimilated Mexican Americans.

Contemporary usage of the term "folk" offers various definitions ranging between references to societal stratification and to an appreciation of traditions and cultural systems. Applewhite describes "folk healing” as "a set of health beliefs and practices derived from ethnic and historical traditions that have as their goal the amelioration or cure of psychological, spiritual, and physical problems” (1995, p. 247). Such positive associations with the term are related to a revival of "culture" in more recent times in which this historical aspect of "folk healing practices" is celebrated as an expression of tradition and worthy of preservation. As Hurdle (2002) describes, rather than being diluted by western assimilation and globalization, preservation of cultural essentials has become a priority facilitating an increased acceptance of practices such as folk/traditional healing (Viladrich \& Abraido-Lanza, 2009).

The institutionalization of CAM has fostered research in the area of folk/traditional healing, which has "blossomed in the U.S. in recent decades, along with the holistic healing movement in the 1970s and subsequent New Age Movement and resulting in supporting evidence on the high use of folk/traditional healers among Latinos in the U.S.” (Hsiao et al., 2006; Viladrich \& Abraido-Lanza, 2009, p. 162). The NHIS 2007 study results reported Latinos’ use of folk/traditional healers an alternative 
treatment option by country of origin: Puerto Ricans (29.7\%), Dominicans (28.2\%), Central or South Americans (23.4\%), Cuban Americans (22.9\%), and Mexican Americans (18.2\%) (Heathcote, West, Hall, \& Trinidad, 2011). However, an understanding of the use of specific folk belief systems and their healers is not yet commonly researched. The use of the generic term folk/traditional healer or curandero is used to refer to healers of numerous, separate belief systems.

One of the few works offering a distinction between folk /traditional systems and healers used was completed by Delgado and Humm-Delgado over three decades ago. The authors determined there were five distinguishable folk/traditional healers used by the three largest Latino sub-groups in the U.S.: 1) spiritist (espiritista), 2) santero and santera (Santería traditional healer), 3) herbalist, and 4) santiguador, and 5) curandero with additional variations of healers found in other Latino sub-groups such as parcheros and sobadores among Central Americans (Delgado \& Humm-Delgado, 1982). Spiritists (espiritistas), santeros (Santería traditional healer), herbalists and santiguadores were utilized by Puerto Ricans; santeros/as were most visible among Cuban Americans; and curanderos were the primary healer in Mexican American communities. Over a decade later, Gloria and Peregoy (1996) report that the three main traditional healing systems among Latinos in the U.S. were: 1) Curanderismo, 2) Espiritismo, and 3) Santería.

\section{Santería}

There are a series of related Yoruba and Central West African based religious forms found in the Caribbean, Latin America, and in the United States. Santería is the Cuban variant of this tradition (Brandon, 1993). Santería is considered one of the 
diasporic manifestations of the African belief system of Ifá which is currently followed by an estimated 100 million people worldwide and constitutes the most populous following among the African Diaspora traditions throughout the Americas (Clarke, 2008; Gonzalez-Whippler, 2001; Holliday, 2008; Murguia, Peterson, \& Zea, 2003; Ojelade et al., 2011). Santería functions as both a religion and health care system with a substantial historical and contemporary role in the U.S. within the various Latino sub-groups and communities with particular visibility in Miami-Dade County (McNeill, Esquivel, Carrasco, \& Mendoza 2008; Pasquali, 1994). Santería is also used traditionally as a tool to cope and heal during times of stress and anxiety (Paulino, 1996; Sandoval, 1979). The word "Santería" emerged as a colonial Eurocentric, derogatory term to distinguish the stigmatized "corrupt” popular expressions of Catholicism vs. Orthodox practices in Spanish colonized Cuba (Wuaku, 2011). Today, it is a loose term, or label, that is assigned by outsiders to the African religious manifestations most associated with the strand of practice from southwestern Nigeria (Wuaku, 2011). Practitioners on the other hand, identify themselves by the dominant tradition they practice: Lukumí (also spelled Lucumí) or Regla de Ocha, Ifá, Abakuá, Palo (and further which rama or branch within the Palo tradition), Arará, Espiritismo, Reglas Conga, etc. Despite differing origins and theological epistemologies (various African influences, European spiritist beliefs, native pre-colonial Latin American and Caribbean Indian beliefs and practices, and the dominant African Yoruba dogma), these independent and varying belief systems are experienced as continuous and overlapping with a reconciliation rooted in the notion of spiritual balance and its relationship to health and illness (Santo, 2010; Sandoval, 2008). Within the individual systems comprising the Santería tradition, physical and mental 
health are not divorced from the metaphysical. An individual's life is viewed as a spiritual phenomenon with healing practices based on the premise of mutual interdependence between the physical and spiritual dimensions (Ortiz et al., 2008).

\section{Santería in Cuba}

Santería as an organized religion emerged in Cuba in the late $19^{\text {th }}$ century as a direct result of the slave trade dating back to the 16th century and the subsequent sociocultural shifts that occurred thereafter (Holliday, 2008). Spanish colonial dominance over Cuba at the turn of the 16th century resulted in the importation of African slaves. For three centuries thereafter, African slaves were an integral part of the Spanish colonial enterprise, working themselves into a social hierarchy constantly in flux, and arriving continuously into the island both legally and illegally carrying their distinct regional cosmologies (Dodson \& Batista, 2008). Cuba reportedly received between 702,000 and one million slaves from Africa over the course of the Atlantic slave trade that ended in Cuba in 1865 with most slaves arriving in the last thirty years (Brandon, 1993, pp. 43). It is within this time period (1500s-early 1900s) where a unique, racially mixed population and highly stratified society developed in colonial Cuba along with a creole culture which facilitated the amalgamation of numerous belief systems.

The Yoruba arrived in the largest numbers. Slave families and communities were purposefully separated upon arrival to the island to discourage the opportunity of collective resistance, which transpired in neighboring St. Domingue (Haiti) between 1791 and 1804. However, despite the Christianization of the slaves by the Spanish Catholic colonizers, the sheer size of the Yoruba population resulted in a significant impact and 
continuity of their belief system. The descendants of those who spoke Yoruba became the Lucumí nation and managed to solidify an identity in spite of attempts to prevent collective action (Brandon, 1993). However, prior to doing so, Brown (2003) discusses the micro-societies that were created in place of natural families (palenques and sanctioned social clubs called cofradias, and cabildos in urban areas such as Havana) consisting of multi-African ethnic groupings which forged a safe space for the transculturation process to occur: an exchange of ideas, customs, cosmology, spirituality, ritual behaviors, etc. Beliefs and customs were shared, retained, eliminated, or modified to retain the essence of each system.

Palenques were secluded communities mostly comprised of escaped slaves, or cimarrones, in the eastern, rural parts of Cuba. Due to the seclusion of the palenques communities, customs and religious beliefs were practiced overtly due to the lack of colonial influence or interference. Cofradias and cabildos nacionales, however, were located in the western, urban part of the island and associated with the Catholic Church. Each held a leader, or "king”, which enacted intermediary governance over the society members. These social clubs participated in public processions and performances on Catholic holy days and saints' feast days. They were also the means by which Yoruba religion was preserved within the clubs' identities; identities which increasingly aligned with the spiritual path followed by the members and less on ethnic composition (Brandon, 1993; Brown, 2003). It was these cabildos and homes of free blacks that took the place of African temples. It is where Yoruba beliefs and rituals were communally practiced and where the African priesthood was reconstituted (Brandon, 1993). 
Due to the persistent socio-cultural-political transformation of Cuba, none of the various African traditions inclusive of the dominant Yoruba could remain static. As Brandon explains, three important changes took place...during the passage from Yoruba to Lucumí religion: syncretism between the orisha and the saints, integration of the separate Nigerian cults into a single religious structure, and the demise of the cults associated with the earth (1993, pp. 76).

Even though links between Yoruba ancestry and Lucumí religion remained, it was possible for people to become "Yoruba” via religious initiation (Brandon, 1993). The late $19^{\text {th }}$ century and first half of the $20^{\text {th }}$ century proved to be a decisive turning point for the religious tradition that would become identified as La Regla Ocha, La Religion Lucumí, and Santería. Much of today’s practice is traced back to the elders practicing in the mid to late 1800s forward which included the last of the slaves imported from Africa and numerous creole Cubans. The Yoruba Ifá corpus and liturgy were recreated and regarded as the ceremonial structure on which Santería is built. The profession of the oriate was birthed, and roles among babalawos (Ifá high priest or "father of secrets"), oriates, and orisha priests \& priests (regarded as santeros/as) were contested, reinterpreted, and redefined. Innovations in the divination and initiation processes took place. Efforts to standardize the practice finally took shape as well, and resulted in a relatively standardized initiatory "modern" model that began to dominate in Havana from the mid-1930s on and was considered universal by the 1950s (Brown, 2003). 
This standardization of practice aligns with the earliest detection of Santería entering the United States and with the Cuban Revolution taking power in Cuba in 1959. The communist regime suppressed religious freedom and was firmly directed towards the Catholic Church and Protestant sects. However, Castro's government gave the AfroCuban religions public recognition as national cultural folk heritage and its practice was offered some sense of tolerance (Brandon, 1993). Therefore, Santería practice has continued up until this day in Cuba and the island remains a popular travel destination to learn about and participate in religious rites and rituals among scholars and practitioners of the belief system.

Santería in the Americas and the United States Santería as practiced today in Miami-Dade County resulted in response to the circumstances of migration and colonial pressures during Cuba’s historic-sociocultural transformation from the $16^{\text {th }}$ to $20^{\text {th }}$ centuries (Sandoval, 2008). Santería entered the U.S. as early as the 1930s with the wealthy and urban educated Cubans, however, its highly visible presence in the U.S. can be traced to the historically catalytic Cuban Revolution (1959-1962) and the continuous immigration of Cubans since then primarily to the East Coast (Holliday, 2008). However, McNeill and colleagues indicate Santería is well documented not only within the U.S. Cuban American community, but "has been noted for its spread across continents and cultures” (2008, p. 70). In addition to its popularity in the U.S. (Miami, Chicago, New York, Los Angeles, and San Francisco), it has a strong presence in the Caribbean (Cuba, Puerto Rico and Dominican Republic) as well as many other Latin American countries (McNeill et al., 2008). Over two decades ago, Skinner 
(1994) estimated that there were over 70,000 Santería practitioners in South Florida. Over a decade ago, Gonzalez-Whippler estimated over a hundred million practitioners in Latin America and the United States which indicates Santería has spread well-beyond its AfroCuban roots (2001).

Santería’s traditions function as anchors to cultural identity. In Latin America, it has demonstrated its resonance with 1) the widespread influence of Kardecian spiritualist beliefs in the late 19th and early 20th century and the distinct "Mexican Spiritualism" popular in the 1920s, 2) the salience of veneration and mythologies surrounding the folk saints, and 3) the prevalent practice of curanderismo which has comparable ritual elements (Ortiz \& Davis, 2008; Sandoval, 1979). In the United States, the tradition has demonstrated its appeal to serve as a mediating institution for Latinos not only of Cuban heritage but all Latino backgrounds (Sandoval, 1979, 2008). Santería has even facilitated a gateway for African Americans to return to their African cultural identity. Walter King was the first African American to be initiated as a santero. Soon thereafter, King began using his African name and adopted a more purely Yoruba based theological structure to his practice.

Within the Santería community in the U.S., much debate continues on the topics of initiation and ritual protocol, and more so, the roles and duties held by the babalawos, oriates, and santeros/as. There remains “contested ritual and political terrains” (Brown, 2003, p. 157). The authority of "who" can do "what" is still heavily challenged as is the "why". Therefore, despite successes achieved in Cuba a century ago or so in regards to standardizing practice, a continuum of variation in ritual practice still exists today. 
Even though folk and traditional healing is evidenced as prevalent among Latinos in the U.S., Zapata and Shippee-Rice (1999) discuss the near impossibility at calculating the number of curanderos in practice in the U.S. because many conceal their identity because of fear of health and legal authorities. They are instead known by word of mouth (Viladrich, 2006). The same complication exists in attempting to ascertain an exact prevalence of Santería healers in the U.S. since they face the same circumstances. Additionally, the African slaves' religious practices were outlawed in Cuba by the Spanish colonizers subsequently causing persecution for any related religious expression. Therefore, Santería is a tradition that even today errs on the side of secrecy due to that history of persecution (Holliday, 2008). However, botánicas are material proof of Santería’s presence as a religion and healing tradition. Through the prevalence of botánicas, access to healers by clients takes place and is unconcealed (Viladrich, 2006). Botánicas are located in areas heavily populated with Latinos and function as "the visible door to the invisible world” of Latino folk healing and Santería practices, and serves as an informal network hub for Latinos as well.

Another tangible indication of the prevalent practice of Santería is the legal recognition it received as the result of the case brought forth by the Church of Lukumí Babalu-Aye regarding religious freedom heard by the Supreme Court. In 1987, Ernesto Pichardo, a Miami santero and oriate, leased and then bought a parcel of land in MiamiDade County in the city of Hialeah to build a church for the communal gathering and practice of Santería (Laycock, 1994). Issues with the Mayor, who had personal interest in the same piece of land, ensued and resulted in the City Council passing a series of ordinances specifically aimed at limiting the practice of Santería rituals, specifically 
animal sacrifices. Pichardo brought suit against the city for violation of the constitutional right to the free exercise of religion. The Southern District Court of Florida heard the case in 1989 and ruled the ordinances were constitutional (Skinner, 1994). The Supreme Court granted certiorari and heard the case in 1992 (Skinner, 1994). The Supreme Court provided a ruling in 1993 and struck down the ordinances by a vote of 9-0 in which the holding indicates "the states cannot restrict religiously-mandated ritual slaughter of animals, regardless of the purpose of the slaughter” (Laycock, 1994). Today, the church not only helps meet the Lucumí community’s spiritual needs by providing resources and services, but aims to provide accurate information on the practice. Membership in the Church of Lukumí Babalu-Aye includes followers located in the United States, Cuba, Puerto Rico, Mexico, Venezuela, Colombia, Ecuador, Guatemala, Panama, Nicaragua, the Dominican Republic, Peru, Trinidad, Jamaica, Italy, Russia, Hawaii, Great Britain, Sweden, and Spain, among the 35 nationalities represented among their members (Church of the Lukumi Babalu-Aye, 2014).

Despite Pichardo’s efforts to consolidate the community of practitioners, Santería remains decentralized and practiced on a more personal level among consortiums. There is not only variation among practice, but in Latinos' level of belief as well, ranging from rejecting Santería beliefs openly but seeking consultations when faced with crisis or stress, to occasionally visiting a botánica, to formal initiation and full participation (McNeill et al., 2008). However, evidence suggests the tradition functions as a subsidiary of formal mental health services. Viladrich and Abraido-Lanza (2009) document the modern-day role of Santeria healers as informal counselors who fulfill the demand for psychological services among diverse subgroups of Latinos. To offer an understanding on 
these fundamental cosmologies of which Santería is comprised, a short discussion on the contributing belief traditions that form its basis is offered.

Ifá

Among the numerous African nation sub-groups, the Yoruba arrived in the greatest numbers towards the end of the slave trade. The heterogeneous Yoruba subgroups from Nigeria, who arrived into the western ports of Cuba in extraordinary numbers in the mid-19th century, came to be known as the "Lucumí”, and introduced Ifá as a belief system. The primary focus in the practice of Ifá is achieving and maintaining balance. In the Diaspora, Ifá is more regularly employed to resolve very specific matters. The tradition in Africa acts far more than a belief system, religion, ideology or philosophy. It is more appropriately a worldview and way of life. Human circumstances in its entirety, including health and illness, are anchored in the guidance and intervention Ifá offers. Application of Ifá cosmology is believed to maintain one’s progress towards balanced, stable, holistic health, and furthermore a good destiny.

Ifá is a monotheistic belief system acknowledging Olodumare as the one God. A pantheon of lesser, but very powerful deities, act on behalf of Olodumare. These orisha are believed to be intermediaries between man and Olodumare and intervening to rectify imbalances indicated through the divination process. The orisha have individual, personified identities that are associated with certain human characteristics, elements from nature, and strengths. These qualities create a sense of relatability towards the orisha and facilitate impressions of fondness. 
Ifá is primarily an oral tradition. The earliest records of the Yoruba way of life and theological structure of their civilization were preserved in Egypt between 6,0005,000 BCE (Salami, 2008). It was believed by the Yoruba people that Ifá was present when God, or Olodumare, created the universe and is privy to the secrets and destinies of all that is in existence, including man. Through the babalawo's divination, believers and practitioners of Ifá can access information and guidance on how to manage life's numerous circumstances (health/illness, career, relationships, obstacles, etc.) that are contained in 256 signs, or odus.

\section{Lucumí}

The Spanish catholic colonizers in Cuba prohibited the African slaves from practicing their Yoruba-based religious tradition and considered the role orisha held in their religion to be idolatrous and sacrilegious (Holliday, 2008). Consequently, the Africans practiced Catholicism overtly and their African faiths covertly. Only after generations of transcultural evolution was there a reconciliation and fusion of cosmologies. However, the Ifá literary corpus is considered the foundation of Santería with its epistemological framework built on the role of the orisha as an essential, defining attribute of this belief system (Brown, 2003). The role of Ifá and the babalawo in the establishment of Lucumí religion in Cuba is significant (Brown, 2003).

As the traditions and practices of Santería evolved, the function of the santero and santera emerged. Santeros/as are individuals who have undergone the ceremony of "hacerse santo", or making saint, which stems from the initiation ceremonies of the orisha priest/priestess in Africa. In Santería however, he or she receives specialized 
knowledge during a modern, standardized initiation process and is apt to divine for clients and practitioners alike by means of the santos (the saints/orisha) versus the babalawos’ divination via Ifá. Divination methods used by santeros/as are obi (4 coconut pieces or cowrie shells) or diloggun (configuration of 16 cowrie shells). Initiation as a santero/a forges the ultimate metaphysical bond and relationship with the orisha in which the initiate devotes a life of service to the orisha in return for a lifetime of guidance and protection (Holliday, 2008).

Another position within Santería that emerged was that of the oriate, who is considered an ordained diviner. Santería also holds a redefined collection of 1) odus which define life's issues, 2) eses verses which became pataki short stories/myths describing the issues, and 3) prescriptions such as ebos or other ceremonies such as cleanings provided to rectify imbalances (Holliday, 2008).

Proselytizing, dogma, and orthodoxy are not features of orisha tradition and no central body information, behavioral norms, or even religious knowledge is in place. Therefore, there is a lack of a governing agent overseeing the correct execution of practice giving way to an individualized preference for religious customs despite efforts to centralize as indicated previously. Debate over rituals, doctrine, authority to perform certain ceremonies and bestow sacred objects, etc., continues today. Undeniably though, functions once exclusive to babalawos were progressively adopted and practiced by oriates and santeros/as (i.e., creating energy pools such as Guerreros, or Warriors.) Leadership roles held by women in Africa, i.e., iyanifá, were dismissed due to the absorption of Spanish, catholic patriarchal values as well. Such transcultural evolution of Santería necessitated such reconfiguration for the survival of its fundamental structure. 
Regardless, the traditional healers, babalawos and santeros/as alike are respected according to the esoteric knowledge they accumulate and by their demonstrated ability to perform the metaphysical rituals (Sandoval, 2008).

\section{Palo}

The first Africans purchased for labor in Cuba arrived from the Kongolese Kingdoms in Central West Africa in the early 16th century (Dodson \& Batista, 2008). From 1760 to 1790, the Kongo and Carabali nations constituted the majority of the Cuban slaves, between 1800-1820 these two groups were still the most populous among the plantation slaves, and the last wave of Kongo migration to Cuba occurred between 1831 and 1867 (Brandon, 1993, pp. 57; Frisvold, 2010). Melting pots of Kongo religious diversity, where myths, legends, and customs were exchanged, were found at the ports of Luanda and Cabinda; were nominated as "Kongo" and "Angola”; and shaped the ideas and practices of Candomble, Umbanda, Kimbanda, Vodou, and Palo Mayombe (Frisvold, 2010, pp. 5).

These central west African groups shared a similar cosmic orientation with the island natives of Cuba, the Taino and sub-Taino Indians, subsequently fusing rituals and traditions that took firm root predominantly in eastern Cuba where the earlier slaves settled (Dodson \& Batista, 2008). This cosmic orientation focused on ancestors, the interconnection of ancestors and the land, communication with spirits, and the understanding that spirits inhabit the world of humans (Dodson \& Batista, 2008). Information is received via divination and revelation but most communication is achieved with spirits of the deceased. 
Palo recognizes a single Supreme Creator, Nsambe or Nzambi. All things animate and inanimate are considered to possess energy and spiritual essence. Natural elements including trees, plants and herbs are relied upon as materials necessary for healing. Emphasis is also placed on the necessary role and relationship with spirits of the deceased which are active in the material world of humans’ present time (Dodson \& Batista, 2008). Spirits of the deceased are invoked and will communicate with the devotees, if not embody one. Communication is achieved by both formal divination procedures as well as through revelation. The tata is the identified leader and godfather of a given practicing community, with the yayi being the 'mother' or godmother.

Sacred objects such as cazuelas ${ }^{2}$ hold materials that generate and harness energy necessary to restore imbalances in the relationships held with all that Nsambe brought into existence (Dodson \& Batista, 2008). Palo work is a vigorous, interactive, energetic, exchange between spirits of the initiated practitioners, generalized spirits of the living dead, and the spiritual power of the tata. Scripted ritual is the core of efficacious power in the religion and needed for the engagement with the spirits. Energies from elements in human time and space must interact with those from supernatural time and space if a given issue is to be resolved with spiritual assistance (Dodson \& Batista, 2008).

Of all the systems discussed in this proposal, this is the tradition with the most limited amount of academic literature. There are numerous ramas, or branches of Palo separated by distinctions in the manner in which the traditions are practiced. Specific information and knowledge pertaining to Palo is revealed strictly through progressive initiations and is a deeply guarded tradition. Blended with roots of a warring people, its

\footnotetext{
${ }^{2}$ A shallow bowl-like container
} 
practice of sorcery or magic, and the harnessing of spirits understood to be "dark spirits" (as in lacking spiritual evolution or light), Palo lends itself to the interpretation of being a "dark" or destructive tradition. Frisvold, one of the very few authors on the Palo tradition and practice, suggests that it is a "religious cult of Creole Sorcery developed in Cuba" (2010, pp. i).

\section{Espiritismo}

In the late 19th century, Kardecian spiritism entered Cuba and was eagerly embraced amidst the political upheaval (Holliday, 2008). It offered a viable "anticolonial" alternative to those who did not favor the dominant African-based traditions and was well received by the white and creole upper and middle classes. Espiritismo, or spiritism is a tradition originating in France through the teachings and writings of educator Hippolyte Leon Denizard Rivail (1803-1869) who used the pen name, Allan Kardec (Holliday, 2008).

The response to conflicting views of science versus religion included the proliferation of spiritualism and spiritism in the 1800s (Holliday, 2008). Kardec distinguished spiritism from spiritualism by determining spiritualism was a general term applied to anyone believing in the metaphysical but not necessarily in spirits. He founded spiritism as an epistemology after applying scientific examination to spiritualism and focused precisely on the belief in spirits. He was concerned with demonstrating the scientific veracity of spirits and their capacity to communicate and visit the historical world of humans (Dodson \& Batista, 2008). 
In espiritismo, spirits contact individuals, more specifically mediums, and transmit knowledge, information, and guidance to them. Additionally, they may embody the medium. In doing so, the medium has granted the spirit access to use them as a vessel at which time the spirit delivers the message personally. Traditionally, communication with the spirits was achieved through séances, groups arranged by Rivail. He systematically documented the manner in which sessions were conducted, the manner in which spirits would deliver information and advice, and documented the actual information the spirits' offered. Kardec published a number of writings. The most notable contribution is five books of the Spiritist Codification, also known as the Spiritist Pentateuch and The Five Fundamental Works of Spiritism published between 1857 and 1868. The first, The Spirits' Book was released in 1857 and has since been translated into 45 languages (Nunez, 2008).

Kardec's writings and his spiritism philosophy were informed by the spirits channeled and interviewed. The spirits were the source of information and helped to establish important principles of communication between the physical and spiritual worlds. It redefined concepts of human suffering, imperfection, and health/illness/disease for followers (Nunez, 2008). The guidance afforded by spirits in this tradition can be considered a form of revelation versus divination. Kardecian doctrine drew from Christianity but Kardec maintained that spirits could be put into different groups or categories as determined by their level of spiritual evolution and their ability to communicate and visit the living.

Kardecian philosophy and teachings disseminated internationally and permeated areas such as Spain, Eastern Europe, the United States, the Caribbean and Latin America 
by the end of $19^{\text {th }}$ century (Koss, 1987). Latinos continue applying the principles of Kardecian espiritismo when interacting with the spirit world. Fundamental practices such as séances are common in today’s Santería tradition but occur in the form of spiritual sessions, or misas.

Santería \& Latino Mental Health Care

Santería provides Latinos a cultural conceptualization of health, well-being, disease, and illness constructing the experiences associated with each, in addition to determining appropriate types of treatment (Holliday, 2008). Folk and traditional healers have historically fulfilled the roles of physician, apothecary, and counselor as well as that of religious and spiritual authority (McNeill \& Cervantes, 2008). Folk healing traditions inclusive of Santería function in much the same way as mental health services, serving as "informal counseling” with spirituality as an added dimension offering explanations as to the "why" of the occurrence of health versus illness (McNeill et al., 2008; Viladrich \& Abraido-Lanza, 2009).

These healers played an important role in society and were held to many of the same standards of practice as modern-day healthcare professionals (Tafur, Crowe, \& Torres, 2009). Folk or traditional healers are said to contain the same four common components that render a formal mental health professional effective: 1) shared worldview, 2) the personal qualities of the therapist, 3) patient expectations, and 4) and use of techniques (McNeill \& Cervantes, 2008). In addition, Jerome Frank has discussed the commonality all healing practices share: “1) an emotionally charged, confiding relationship with a healer, 2) a healing context in which the therapist has the power and 
expertise to help and holds a socially sanctioned role to provide services, 3) a rationale or conceptual schema to explain problems, and 4) "a ritual or procedure consistent with the treatment rationale” (McNeill \& Cervantes, 2008, p. xxvi). These common qualities create the most culturally specific context for service delivery theoretically rendering the services provided by folk or traditional healers culturally congruent to their clients’ understanding of health and illness and thus, highly effective (McNeill \& Cervantes, 2008).

Doctoral research completed by Antonio Santi, Jr. explored the validity of Santería as a mental health care system in Miami-Dade County by comparing Santería healers to formal mental health professionals (MHP) (1997). Data was collected on the experiences and outcomes of visiting both MHP and Santería traditional healers. Results indicated Santería practitioners, who comprised the sample, preferred their traditional healers over MHP due to the MHPs' lack of understanding and sensitivity. It is unclear what the MHP lacked understanding and sensitivity towards; however, Santi’s work offered indication that Santería serves a comparable, albeit informal, mental health function among Latinos in Miami-Dade County. This study distinguishes itself from Santi's prior work by aiming to gain a clearer understanding of the mechanisms that compel Latinos to seek Santería as a mental health support and more specifically, the characteristics of Santería that appeal to Latinas, but within the context of mitigating with the psychosocial sequelae of the cancer experience.

\section{Conceptual Model}

The conceptual model of the proposed study is the Internal Structure of Local 
Health Care Systems, a component of medical anthropologist Arthur Kleinman’s Explanatory Model of Health and Illness (EM) (1980). The Explanatory Model was developed by Kleinman to account for the subjective, cultural context of the health, illness and healthcare experience of psychiatric patients in Asia (1988). The Explanatory Model stipulates that all groups possess "culturally-constructed" explanations of health, illness, and treatment which define their health seeking behavior (Rahill, 2008).

A specific concept within the Explanatory Model is a group's Internal Structure of Local Health Care System (ISLHS). The ISLHS is comprised of the professional, folk, and popular sectors. The professional sector is comprised of the formal healthcare services (i.e., western, biomedical conventional professionals.) This includes professional mental health services such as psychiatrists, psychologists, counselors, support groups, and research clinical trials and interventions. The folk sector is comprised of the nonprofessional, non-bureaucratic healing practices specialists which treat based on both secular and sacred practices (Kleinman, 1980). This includes herbalism, surgical and manipulative treatments, exercise, indigenous but non-sacred rituals, folk psychotherapy, as well as sacred/spiritual healing rituals and ceremonies. The popular sector is the culture-laden area indicative of the popular culture and social reality of the individual which links the boundaries between the professional and folk sectors offering the room to choose entrance and exit into either other sphere (Kleinman, 1980). It is the individual, family, social, and community based arena in which illness is first defined and health care activities initiated by individuals. 
Figure 1- Internal Structure of Local Health Care System

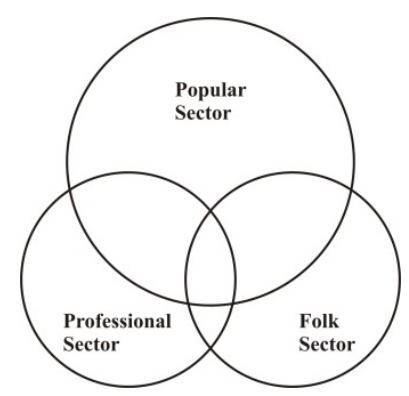

The popular sector functions as the most immediate determinant of care.

Individuals first encounter disease in the family: perceiving symptoms, labeling and evaluating the disease, deciding what to do and engaging in specific health seeking behavior (Kleinman, 1980). Beliefs and values about illness are utilized as part of the popular culture forming the basis of attitudes shared by a community. The popular sector sets the parameters for the experience of disease/illness and acceptable forms of treatment options. Once the individual, or patient, decides to move into the professional or folk sector, they encounter difference sets of beliefs and values in the cognitive structures of the practitioners each in respective sector (Kleinman, 1980).

The notion of an internal structure of local health care systems provides a framework for understanding the cognitive process of health seeking behavior. Kleinman argues that professionals organize health care for lay people, but typically lay people activate their health care by deciding "when and whom to consult, whether or not to comply, when to switch between treatment alternatives, whether care is effective, and whether they are satisfied with its quality” (1980, pp. 51). Furthermore, Kleinman asserts that when people finally resort to folk or professional practitioners, “their choices are anchored in the cognitive and value orientations of the popular culture” (1980, pp. 50). 
The popular sector is that space defined by the culture that the patient decompresses, evaluates, and strategizes their next move in regards to treatment. It is the space where the fundamental attitudes and motivation for health seeking behavior exists creating the gateway for use of both formal, allopathic professional services and indigenous folk healing traditions that exist among Latinos. For this proposed study, this translates into evaluating if Santería is a commonly accessed folk tradition by Latinas with cancer thus existing in the popular sector as well. As a treatment modality located in the popular sector, it theoretically reflects the general attitudes held among Latinas towards Santería as an acceptable and fundamental culturally congruent mental health treatment option. The study will offer insight into the distinct qualities of Santería that 1) distinguish the practice as culturally congruent and 2) underlie the motivation of Latinos to seek this service.

In sum, the proposed study is based on a conceptual model where individuals as patients demonstrate the attitudes of the popular culture through their health seeking behavior. Using ISLHS model proposed by Kleinman in his Explanatory Model of Health and Illness (1980) to build upon, this research serves to understand the attitudes of Latinas living with cancer towards the Santería as mental health service option and exploring Santería's effect on the psychosocial sequelae experienced by the women.

\section{Research Objectives}

The exploratory study will examine the impact of Santería on the mental healthseeking behavior of Latinas living with cancer in Miami-Dade County by means of the following two aims and three questions as modeled in Appendices. 
Aim 1: Determine the attitudes of Latinas living with cancer towards Santería as an informal mental health support that assists in coping with the psychosocial sequelae of living with cancer.

Aim 2: Explore whether Santería offers Latinas effective mental health support that assists in coping with the psychosocial sequelae of living with cancer and if so, how?

\section{Research Questions}

Question 1: What effect does Santería have on the psychosocial sequelae of Latinas with cancer?

Question 2: What are the mechanisms of this effect, which make Santería helpful or harmful to coping with the psychosocial sequelae of living with cancer? Question 3: What are the characteristics of Santería that distinguish it from formal mental health services? 


\section{CHAPTER 3}

\section{Research Design and Procedure}

\section{Qualitative Exploratory Research Strategy}

The purpose of this exploratory study is to examine the impact of Santería on the mental health seeking behavior of Latinas living with cancer in Miami-Dade County. To accomplish the two aims specified in the last chapter, the study will be conducted in two stages using qualitative approaches to data collection and analyses: 1) interviewing and a 2) focus group. Through the availability of IRB approved printed material and word of mouth networks throughout the community, Latinas with the experience of cancer were recruited from community based organizations and botánicas and interviewed, which served as the primary mode of data collection. A focus group with Latinas that were interviewed was held for validation. A well-respected leader in the Santería community was interviewed to further triangulate findings.

A qualitative approach was appropriate for this early or 'exploratory’ stages of research (Silverman, 2000). The empirical literature examining the mental health seeking behavior of Latinas living with cancer was limited. Stebbins (2001) indicated an exploratory approach as most appropriate in such situations. Methodologically, a modified grounded theory approach was employed. This allowed for the development of theory that was grounded in the experience of respondents (Strauss \& Corbin, 1994).

The research design provided a base of in-depth data regarding the motivation of Latinas living with cancer to seek Santería services and how Santería may or may not have related to coping with the psychosocial sequelae of the disease. The design offered 
the opportunity to better understand specific health seeking behavior among Latinas with cancer.

Recruitment

Participants were recruited from 1) community-based organizations providing formal cancer psychosocial and mental health support programs and 2) botánicas in Miami-Dade County. The community agencies include: La Liga Contra El Cancer (The League Against Cancer) and the Cancer Support Group of Greater Miami. These recruitment sites were selected because they are: (a) well-recognized providers of cancer support care in Miami-Dade County and (b) a large number of the clients who receive their services are Latinas.

\section{Community-Based Organizations}

At each organization, printed flyers with information about the study were posted as well as distributed by agency staff to their patients. The researcher's contact information was listed on the printed material and available through the agency staff. In addition, the researcher attended support groups, events, and activities at each location as per the schedules put forth by each location. The agency staff introduced the researcher at these formal functions. As the researcher approached or was approached by individual women who were interested in learning more, she was asked to join the researcher in a removed, private area where the details of the study were explained. When interest in participation was indicated, the researcher determined if the woman was eligible by asking screener questions based on the eligibility criteria. The women who were eligible 
and prepared to sign the consent form did so with the researcher at that time and the researcher noted participants' contact information. The women who instead requested time to consider participation were asked to provide their contact information and they were offered the researcher's contact information as well. The researcher followed up each woman that had requested time within a week's time.

\section{Botánicas}

Since the literature indicates that Latinos underutilize formal mental health services, participants were recruited through a number of word of mouth networks accessed at shops identified as botánicas found throughout Miami-Dade County. Botánicas are the socially visible entry points to Santería. Rapport was established with both the botánicas’ staff as well as with the folk healers affiliated with each shop. The investigator identified members of the botánica’s Latina client base who met the eligibility criteria for this study. Working with the staff and folk healers facilitated access to participants. These botánicas served as locales to disseminate information on

the study, facilitate recruitment, and offer deeper penetration into the community of those accessing Santería services.

Printed material was left at participating botánicas, made available and distributed among their patrons. Additionally, botánica staff and the healers affiliated with the shops were asked to refer Latina clients with cancer that met inclusion criteria. The researcher contacted them via phone, described the study to them, and offered an invitation to participate. Those interested were asked the screener questions to confirm eligibility. When eligible, a time and location of choice convenient to the participant was scheduled 
for a brief personal introduction and for the signing of the consent form. At that time, in person, the researcher answered any questions and attended to any concerns the participant expressed.

Stage One Data Collection: In-depth Interview

Data was primarily collected using in-depth interviews with the Latinas living with cancer. The overall study objective during this stage of data collection is focused on collecting information about the Latinas’ attitudes towards Santería and the motivations for its use.

An incentive of \$20 was provided upon completion of the interview as indicated in the consent. The interview was offered and conducted in English or Spanish contingent on the language preference indicated by each woman. Average length of the interview session was approximately one hour. The researcher had space at Florida International University to conduct the interview sessions, but ultimately the participants' time and location of choice was honored. Interviews were held at participants' homes, workplace, or public venues such as a Starbucks. If written informed consent had not been completed, it was done so here prior to beginning the interview. Participants were also asked if they were interested in participating in the Stage Two focus groups. A digital audio recorder was introduced at the start of the session and used to audio-record the interview.

Interviewer Training

The study researcher will conduct the interviews. Over the course of 14 years, she 
had been a member of the research staff on various federally funded studies conducted by the Department of Epidemiology \& Public Health of the Miller of School of Medicine, University of Miami, and the Center for Research on U.S. Latino HIV/AIDS \& Drug Abuse at Florida International University. As research staff, she participated in standardized training at the start of each study and periodic trainings held on-site throughout the course of each study until completion. This included intensive training on adhering to research protocol, preparing for data collection activities, English and Spanish face-to-face interviewing with vulnerable populations regarding sensitive topics, leading and moderating groups focused on sensitive topics with vulnerable populations, establishing rapport, listening, recording and processing simultaneously, creating comfortable pauses, offering redirection, use of probes and drawing out responses, controlling body language and reactions, maintaining the qualities of politeness, relateability, and cultural sensitivity (Berg, 2001; Padgett, 1998). From years of interviewing study participants, the researcher has become well-experienced working with Latino populations, as well as in detecting on the part of the participant incomprehension or misunderstanding, topic avoidance, deliberate distortion, etc., all necessary skills needed to ensure a successful interview session (McCracken, 1988). Additionally, the researcher is part Latina, fluent in Spanish, and has conducted interviews in Spanish. The researcher's extensive training and experience in qualitative interviewing techniques facilitated an awareness and avoidance of introducing bias into the data analysis process. Additionally, the researcher regularly sought feedback from her advisor to further ensure bias was minimized. 


\section{Interview Guide}

Interviews are an especially effective method of data collection when the researcher wants to understand the perceptions of participants as well as how certain meanings are attached to phenomena or events (Taylor \& Bogdan, 1998, pp.98). The interview guide for this study elicited responses that provided access to understanding the Latinas’ experience of using Santería as an informal support and what impact such an experience has on the psychosocial sequelae of living with cancer.

A semi-structured protocol was used composed of open-ended, predetermined topics informed by the literature and the ISLHS model to generate discussion on the Latinas’ experience with Santería. Prompts were used to facilitate discussion and maintain focus on the matters of interest with minimum input from the interviewer. The second part of the guide included survey questions that collect demographic and diagnostic information (i.e., birthplace, identification with Latino subgroup, years in the U.S., cancer diagnosis, treatment history, etc.)

\section{Transcription of Audio Recordings}

The researcher transcribed the in-depth interviews and the focus groups’ digital recording verbatim. The researcher then reviewed the transcripts by listening to the audio recording while reading the documents to confirm their accuracy. Maintaining the transcript in the original language provided benefits in terms of maximizing data integrity, as well as saving time and resources. Selected portions of interviews included in the dissertation manuscript were translated to English by means of the translation/back translation method (De La Rosa et al., 2012). The Spanish components were translated to 
English by the interviewer, and then back translated into Spanish by a second, independent translator.

Stage One Data Analysis

Computer Assisted Qualitative Data Analysis Software (CAQDAS)

ATLAS.ti (ver. 7) was used to organize and aid in the coding of the text and the researcher's thoughts and memos (Allan \& Skinner, 1991). It also supported the conceptual diagramming that facilitated the identification of relationships and patterns between concepts via its "network analysis” function.

\section{Codes \& the Coding Process}

Analysis began with the process of coding the data. Coding is "deriving and developing concepts” from the raw data. Codes are the labels given to these concepts derived through the coding process (Corbin \& Strauss, 2008, pp. 65). During open coding, one combs through the data, breaking the data into short, meaningful quotations that can be labeled with one or more codes. The data was coded liberally to allow for as many distinct categories as possible to emerge (Ford, Oberski, \& Higgins, 2000). The data was also coded as per predetermined categories informed by the literature. These "a priori” theoretical codes were compared to the concepts that emerged from open coding. Those theoretical codes found to relate, and could be attached to transcript quotations, were supported as being grounded in the raw data and thus, earned their way into the analysis (Corbin \& Strauss, 2008). The a priori codes not attached to quotations in the transcript were discarded. Each transcript was read in its entirety. 
The researcher initiated a first pass of coding in which evidence of theoretically informed a priori codes in the text were sought and open coding was completed. To increase the reliability of the data, the researcher and an additional researcher met to separately code transcripts. Given the small sample of the study, it was determined comparing two transcripts’ coding for agreement was an adequate inter-rater process to promote rigor and reliability (Armstrong, Gosling, Weinman, \& Marteau, 1997). Each coded "liberally" to identify as many concepts and categories as possible (Ford et al., 2000). They met thereafter to discuss codes and themes, the accuracy and consistency in code usage, and coding strategies (Ford et al., 2000). Additionally, the researcher met with committee members to discuss her analysis activities and monitor coding procedures and outcomes.

\section{Constant Comparison Analysis}

The analysis method of constant comparison was used to compare for incidents of concept and category similarities versus differences among the various transcripts (Corbin \& Strauss, 2008). Data collection completion did not determine the onset of analysis. Instead, analysis began as transcripts became available.

Each transcript was coded as it came in and then compared to earlier transcripts to compare categories or themes. The researcher applied the constant comparison method by looking for evidence of the codes and categories that emerged from each transcript in prior and subsequent transcripts. New codes and categories that emerged later in the analysis were applied to earlier transcripts. This ensured each code was applied uniformly throughout the analysis and did not drift away from its intended use. 
The second stage of the constant comparison method focused on significant code refinement and the identification of more abstract or higher level codes. The researcher examined if concepts or categories could be absorbed under other categories, or if they needed further subdivision (Dye, Schatz, Rosenberg, \& Coleman, 2000). Relationships between the concepts were noted. This consolidation of codes and categories moved the analysis towards the identification of overarching, more abstract, themes and patterns. The third stage of constant comparison moved toward development of themes and outlining theory. The fourth stage of constant comparison was the diffusion of findings through the write-up.

\section{Theoretical Memos}

Throughout the coding process, the researcher used the technique of memoing and recorded her notes as ideas regarding the data that emerged. Memoing in grounded theory has a distinct significance since these notes are the "running logs" of the researcher's analytic thinking and stimulate what is written as a final analysis (Corbin \& Strauss, 2008, pp. 108). Code notes identifying the code labels and their meanings as well as theoretical notes chronicling reflections and thoughts about the concepts, relationships between concepts emerging and theoretical propositions were two forms of memoing performed. Integrated in Atlas.ti software is a memo function which facilitated linking such memos directly to the text during analysis. 


\section{Theory Development}

Once most transcripts had undergone open and theoretical coding, a more selective coding technique was used in which the analysis moved beyond a level of description to one of theory development. The main categories, or themes, that emerged were integrated into an increasingly unified theoretical explanation with a central concept (Corbin \& Strauss, 2008). This pass at coding was more advanced and involved identifying and linking together abstract concepts, patterns, and relationships into a theoretical whole.

Negative case analysis, or the analysis of cases that do not fit and are instead inconsistent with the emerging pattern, was achieved. Finding cases that challenged the emerging themes served to assess the legitimacy of such themes and explore further the contradictory concepts. Negative case analysis provided a richer understanding of the dimensions of a concept (Corbin \& Strauss, 2008).

Conceptual diagrams were constructed to visually "pull it all together” and further make sense of the data "in respect to an emerging theory” (Trochim \& Donnelly, 2007). These visual aids further contributed to the refinement of theory by delineating the relationships between the more abstract concepts and the concepts grounded in the participants’ words. Diagrams offered the reviewer a visual flow of logic and consistency (Corbin \& Strauss 2008).

Stage Two Data Collection: Focus Groups Member Checking

The purpose of member checking was to present and discuss the findings from 
analysis up to that point to confirm that the relevant concepts were captured, clarify results, allow respondents to comment on findings, and enhance the overall rigor of the study. Additionally, member checking allowed respondents to provide additional insights and nuances to findings (Morgan, 1997; Wolff, Knodel, \& Sittitrai, 1993). Therefore, a final verification stage was completed consisting of a focus group with participants from Stage One.

A focus group offered an additional dimension of data collection that was distinct from the in-depth interview. The group provided the opportunity to observe interactions between participants and group dynamics, and the sharing of specific attitudes and experiences. The focus group also offered insight about underlying normative group assumptions (Berg, 2001, pp. 115). However, focus groups in general present some disadvantages, including participants' unwillingness to discuss sensitive topics in a group setting and their concern over lack of confidentiality. The facilitator's training and background enabled her to promote discussion and drew out the more reserved participant. Also, she was able to lead a discussion of confidentiality and reassure participants regarding the sessions’ confidentiality.

\section{Training of Focus Group Facilitator}

The researcher conducted the focus group session. As previously indicated, she had received extensive training as a part of research staff for federally funded studies at the University of Miami and Florida International University. This included leading and facilitating focus groups. Adhering to protocol, managing group dynamics between four to 12 participants, discussing sensitive topics, and working with vulnerable populations 
were some of the specific skills she possessed that qualified her to facilitate the focus group.

\section{Sample Selection \& Protocol}

The purpose of the focus group was to obtain participant feedback on the researcher's initial findings relating to central concepts, major themes, and the relationships between them as determined from Stage One data. These topics formed the basis of the protocol for the focus group and allowed a select, diverse group of participants to comment on their accuracy and meaning and gain additional feedback used in the final analysis. Stage Two served strictly to validate findings, not generate new data.

The sample for the focus group was drawn from the Latinas who participated in Stage One and who previously consented to such Stage Two participation. The researcher selected women with varying answers to the interview questions. The focus group session was scheduled when four women indicated availability and their ability to attend. The group was limited to four women since clarification, discussion of differences in views, and identification of individual voices in the recording becomes increasingly difficult in larger groups (Barbour, 2007). Careful notes were taken regarding refusal to participate in Stage Two. For the women refusing participation, demographic characteristics and characteristics of the individual transcript were compared and discussed in the final paper. Upon a refusal, the researcher proceeded with contacting another participant with characteristics that most closely resembled those of the woman originally selected who had refused. 


\section{Stage Two Measurement \& Analysis}

The focus group was conducted at FIU and was approximately two hours. An incentive of \$20 was provided at the end of the group session to each participant. The group was audio-recorded and transcribed using the same procedure explained in Stage One.

Since this stage served to obtain feedback from the participants regarding the codes generated in Stage One, the analysis focused on linking quotations from Stage Two transcripts to the major concepts that emerged from Stage One. Transcripts were read thoroughly to identify the quotes regarding the major concepts and codes from Stage One and served to clarify their meaning. Analysis in this stage also offered the opportunity to make any necessary changes to the concepts and codes that may were unclear. The analysis for Stage Two was simpler and more; it verified the accuracy of the researcher's interpretations of Stage One data (Padgett, 2008). These findings were presented to the committee for discussion and further feedback before proceeding towards final analysis and reporting major conclusions. 


\section{CHAPTER 4}

\section{Findings}

This chapter provides a description of the major themes and subthemes that emerged from interviews. As a result of the comparative grounded theory process employed, the interview guide evolved as data emerged from the early interviews creating 13 new questions that were added to the initial seven. Forty-five higher level codes were created via multiple passes of coding beyond the initial six a priori codes. Through multiple readings and constant comparison, these codes were further refined and consolidated for a final count of 39 high level codes. No new codes emerged after the twelfth interview. Table 1 describes the participant profiles. Table 2 provides each participant’s number, age, and fictitious pseudo-name selected by each woman as a reference for the quotes provided. The quotes are presented in their raw data form, therefore, the Spanish speaking participants’ quotes are supplied to ensure their authenticity is retained before offering the English translation that was achieved using the translation back translation method.

\section{Sample \& Eligibility Criteria}

A purposive sample of 15 women yielded sufficient demographic variation in the sample as well as varying views on Santería and produced a manageable volume of data (Patton, 2002). The sample for this study consisted of Latinas ages 22 to 79 residing in Miami-Dade County who met the following eligibility criteria: (1) self-identify as Latina, (2) received a cancer diagnosis (any stage/any site), (3) 18 years of age or older, (4) used the services of a Santería healer at least once since diagnosis, and (5) was able to give 
informed consent. Since this study was focused on the psychosocial sequelae affecting Latinas after a cancer diagnosis, and not on pre-existing conditions, a prior history of depression or a mental illness served as an exclusion criterion.

Maximum variation in sampling demographic categorical areas (age, education, SES, foreign/native born, Latino subgroup, and health insurance) within the sample of Latinas with cancer was sought to maximize heterogeneity bringing particular value to the common themes that emerged from such variation (Patton, 2002). The probability of females developing invasive cancers is most significant when she reaches the age of 40 and subsequently comprised the majority of the sample. Therefore, included in the sample were three women under the age of 40 so the experience of younger women was captured as well (American Cancer Society, 2012a). However, Latinas under the age of 18 were not included since they are considered a pediatric oncology population facing different circumstances (Patenaude \& Kupst, 2005). According to the 2010 Census, the median income among Hispanics/Latinos in Miami-Dade County was \$41,152 therefore $\$ 40,000$ was used to distinguish between lower SES and higher SES (U.S. Census Bureau, 2012). Country of birth was recorded (foreign vs U.S. born). Living in the United States for five or more years typically serves as a proxy of acculturation, and was used as such in this study to distinguish low versus high acculturation for those foreign born participants (Fitzgerald, 2010). All participants had lived in the U.S. for over five years. There were also distinctions made between cancer sites, initial versus re-diagnosis, and treatment.

Additionally to accomplish maximum variation within the sample regarding the degree of Santería utilization, three subsamples were included: (1) casual clients, (2) 
practitioners, and (3) healers. Latinas who sought services of divination and spiritual intervention strictly to attend to circumstances that arise without converting to the faith were categorized as “casual clients”. Latinas who have participated in any of the various levels of initiation ${ }^{3}$ (pre- or post-diagnosis) were categorized as "practitioners”. Latinas who have reached the highest levels of initiation in which they are considered "ritual specialists»"${ }^{4}$ and eligible to provide spiritual services to others were categorized as "healers".

Table 1

Participant Profiles

\begin{tabular}{|c|c|c|c|c|}
\hline & & $\begin{array}{l}\text { CASUAL } \\
\text { CLIENT }\end{array}$ & PRACTITIONER & HEALER \\
\hline \multirow[b]{2}{*}{ AGE } & $<40$ & 004 & 001; 012 & \\
\hline & 40 \& over & $\begin{array}{l}\text { 003; 005; 011; } \\
014\end{array}$ & 006; 007; 008 & $\begin{array}{l}\text { 002; 009; 010; } \\
013 ; 015\end{array}$ \\
\hline \multirow{2}{*}{$\begin{array}{l}\text { HOUSEHOLD } \\
\text { INCOME }\end{array}$} & $<\$ 40,000$ & $011 ; 014$ & 001 & $002 ; 009 ; 010$ \\
\hline & $\$ 40,000$ \& over & 003; 004; 005 & 006; 007; 008; 012 & $013 ; 015$ \\
\hline \multirow{3}{*}{$\begin{array}{c}\text { HIGHEST } \\
\text { LEVEL OF } \\
\text { EDUCATION } \\
\text { COMPLETED }\end{array}$} & $<$ High School & 011 & & $002 ; 013$ \\
\hline & HS Graduate & $004 ; 005$ & 007 & 010 \\
\hline & College & 003; 011; 014 & 001; 006; 008; 012 & 009; 015 \\
\hline \multirow[t]{2}{*}{ BORN } & Foreign & $\begin{array}{l}\text { 003; 004; 005; } \\
011 ; 014\end{array}$ & 006; 007; 012 & $\begin{array}{l}\text { 002; 009; 010; } \\
013\end{array}$ \\
\hline & U.S. born & & $001 ; 008$ & 015 \\
\hline \multirow{2}{*}{ INSURANCE } & Insured & $\begin{array}{l}\text { 003; 004; 005; } \\
011\end{array}$ & 001; 006; 007; 008 & $\begin{array}{l}\text { 002; 009; 010; } \\
013 ; 015\end{array}$ \\
\hline & Uninsured & 014 & 012 & \\
\hline \multirow[b]{2}{*}{$\begin{array}{l}\text { YEARS IN THE } \\
\text { US }\end{array}$} & $<5$ Yrs & & & \\
\hline & 5 Yrs or more & $\begin{array}{l}\text { 003; 004; 005; } \\
011 ; 014\end{array}$ & $\begin{array}{l}\text { 001; 006; 007; } \\
008 ; 012\end{array}$ & $\begin{array}{l}\text { 002; 009; 010; } \\
\text { 013; } 015\end{array}$ \\
\hline
\end{tabular}

\footnotetext{
${ }^{3}$ Initiations are rituals of ordination that show membership in and commitment to the faith and to the religious community (Mason, 1994).

${ }^{4}$ Ritual specialists are recognized in the religious community as possessing the knowledge, training, and capacity to spiritually intervene for clients
} 


\begin{tabular}{|c|c|c|c|c|}
\hline \multirow{4}{*}{$\begin{array}{c}\text { LATIN } \\
\text { SUBGROUP }\end{array}$} & Colombia & 005 & 007 & \\
\hline & Peru & 004 & & \\
\hline & Cuba & 003; 011 & 001; 006; 008 & $\begin{array}{l}\text { 002; 009; 010; } \\
013 ; 015\end{array}$ \\
\hline & Nicaragua & 014 & 012 & \\
\hline \multirow[t]{2}{*}{ DIAGNOSIS } & Initial & $\begin{array}{l}\text { 003; 004; 011; } \\
014\end{array}$ & 007; 008; 012 & 009; 010; 013 \\
\hline & Re-diagnosis & 005 & $001 ; 006$ & $002 ; 015$ \\
\hline \multirow[b]{3}{*}{ CANCER SITE } & Breast & 003; 011 & 001 & $\begin{array}{l}\text { 002; 009; 010; } \\
013\end{array}$ \\
\hline & $\begin{array}{c}\text { Gynecological } \\
\text { Ovary/Cervical/uterus }\end{array}$ & 004; 005; 014 & & 015 \\
\hline & Other & & $\begin{array}{l}006 \text { (Non- } \\
\text { Hodgkins } \\
\text { lymphoma \& hips) } \\
007 \text { (Colon); } \\
008 \text { (Colon); } \\
012 \text { (Gallbladder) }\end{array}$ & \\
\hline \multirow{5}{*}{ TREATMENT } & Completed & 004; 011; 014 & 006; 007; 008; 012 & $\begin{array}{l}\text { 002; 009; 010; } \\
015\end{array}$ \\
\hline & On-going & $003 ; 005$ & & \\
\hline & $\begin{array}{c}\text { Undetected after DX } \\
\text { but before TX began } \\
\text { (believed to be due of } \\
\text { folk healing) }\end{array}$ & & 001; 007 & 013 \\
\hline & Used/using CAM TX & 003 & & \\
\hline & $\begin{array}{c}\text { Current concerns but } \\
\text { not receiving TX }\end{array}$ & & & 002 (lump) \\
\hline
\end{tabular}

Table 2

\begin{tabular}{|c|c|c|}
\hline Participant \# & Age & Pseudo-name \\
\hline 001 & 38 & Eddie \\
\hline 002 & 40 & Gitana \\
\hline 003 & 52 & Simone \\
\hline 004 & 22 & Andrea \\
\hline 005 & 53 & Mafe \\
\hline 006 & 53 & Caridad \\
\hline 007 & 52 & Sirenita \\
\hline
\end{tabular}




\begin{tabular}{|c|c|c|}
\hline 008 & 57 & Mariposa \\
\hline 009 & 79 & Lidia \\
\hline 010 & 74 & Cristina \\
\hline 011 & 77 & Oneida \\
\hline 012 & 34 & Mariela \\
\hline 013 & 64 & Madre de Agua \\
\hline 014 & 54 & Elena \\
\hline 015 & 49 & Fatima \\
\hline
\end{tabular}

Themes

As described in chapter 3, the initial a priori codes used were informed by the literature and identified in the transcript (Corbin \& Strauss, 2008). There was evidence found in the data, and all of the a priori codes were attached to quotations and earned their way into the analysis. They were further subdivided into higher level codes as analysis progressed.

The remaining codes emerged from the data set via the process of open coding. Transcripts were combed through and broken into short, meaningful quotations that were labeled with one or more codes. Open coding warranted numerous reviews of the transcripts. On first pass, a concept was identified and a code created to label it. Progressing through the transcripts, the different aspects of the same concept were labeled with distinct codes. The constant comparison method was employed for a back and forth process of checking earlier transcripts and codes to ensure capturing all of the relevant data throughout the numerous transcripts, and to ensure the codes' meanings remained consistent throughout. This process was undergone until all relevant concepts 
and the distinctions within the concepts were satisfactorily identified. More complex codes were unpacked to identify the multiple more abstract concepts embedded within. These higher level codes were then grouped into the three major themes presented below: 1) Participants' Experience with Folk Healers, 2) Influence of Santería on the Cancer Experience, and 3) Participants' Experience with Conventional Healthcare and Mental Healthcare.

\section{Participants’ Experience with Folk Healers}

Coding the experiences the participants shared with the folk healers quickly delivered a distinction between what was indicated as 1) trust in the healer and 2) a personal relationship with the healer. “Ability to heal::folk healers”, "personal relationship::folk healers", and "trust::folk healers” were the codes that built the themes reported in this section on folk healers. Eleven women had used Santería services prior to their diagnosis and four women were referred to Santería folk healers by family or friends (Mariela, Elena, Mafe, and Simone) as a result of being diagnosed with cancer. Ten of the 11 women who had received prior services had an established relationship with a healer. For those who sought services for the first time, Mariela and Elena have remained practitioners and continue to see the healer. All but Mafe and Simone have maintained a very personal relationship with the folk healer with trust and rapport building immediately beginning at the first encounter.

We're not here just to heal you physically. We have to heal you emotionally, mentally, physically, and spiritually. You have to be able to heal the person emotionally, mentally, physically, and spiritually. And that would make you a 
healthy person... that's the difference between going to a regular Dr. that only cares about your damn toe versus a healer that cares about all of those aspects of healing. (Gitana, healer)

\section{Trust in Folk Healers}

Discussion surrounding the folk healer provided a clear indication that all of the women trusted these individuals and their capacities. They were seen as functioning with some similarities to medical doctors in how they approached determining causes and treatment. The healers were undoubtedly credible in the eyes of these participants anchored primarily in a shared understanding that the healers have a "gift", an innate ability and destiny to heal which is granted and guided by a higher power described as spirits of the dead, orisha or santos, God, and/or the Universe. Sirenita explained, "Con mi padrino es algo nato. Es algo que el trae en su sangre, en su ser, un “don” que él tiene... "Don” es un regalo de Dios. [With my godfather it’s something innate. It's something that he has in his blood, in his being, a "don" that he has... "Don" is a gift from God.] The women also trusted in these higher powers as the intervening sources. Lidia explained, “Tengo fe en la religión Lukumi y en las orisha y en la información que me iban a dar." ["I have faith in the Lukumi religion and in the orisha and in the information they were going to give me.”]

The most initial trust the participants placed in the healers was generated from a close family or friend's support and recommendation of the healer. Santería services are made available via word of mouth and referrals. Most of the women in the sample were not raised practicing this belief system, so they were referred to it by someone personally 
known to them. So upon contacting the healer, the participants had already been assured of the healer's competence and ability by their friends and family who had previously experienced tangible positive results. In one regard or another, the healers had already proven themselves to the participants and possessed a sense of credibility.

Yo estaba aferrada que yo iba a salir bien con mi padrino, que lo que el hiciera, así me hubiera ha mandado comer estiércol yo lo hubiera hecho porque yo tengo fe. Creo en los santos. Creo en ellos. Creo en mi padrino. Sé que mi padrino a mí nunca me va, nunca, nunca me va abandonar y nunca me va engañar. [I was clinging (to the belief) that I was going to be alright with my godfather, that what he would do, if he would've ordered me to eat feces I would've done so because I have faith. I believe in the saints. I believe in them. I believe in my godfather. I know that my godfather will never, never abandon me and will never deceive me.] (Sirenita)

\section{Personal Relationship with Folk Healers}

Most of the women felt a deep sense of personal commitment on the part of the folk healer. The folk healer was considered, in addition to family and close friends, as someone she can go to and divulge completely and honestly her most sincere thoughts and feelings on her health status. All of the women felt they could cry and express the fears and concerns they experienced. Participants also valued highly the availability of the healer. Women felt they could contact, interact, and access services far more liberally with their healer. They perceived the healer to be more personally invested in her and her 
contentious struggle against the illness. There was a sense that the healer was fighting alongside her and bore a personal responsibility for the woman's wellbeing. Referring to her Godfather on hearing she was diagnosed with cancer:

Yo vi primero que nada se le aguaron los ojos por que el ser humano le tiene mucho miedo a esa palabra. Y inmediatamente lo vi con una fortaleza y él me dijo, "Mi ahijada de esta tu no queda. Pero de todas maneras vamos a ver lo que dice el caracol.” [I saw that before anything he’s eyes became watery because any human are very afraid of that word (cancer). And immediately I saw him with such strength and he said to me, "My goddaughter, you will not die from this, but anyway let's see what the shells say.”] (Lidia)

You have to be able to hug that person. You have to be able to say, "You know what, you're not alone,” whether I'm a healer or not, "You're not alone.” You know? You can pick up the phone and call me and tell me you're afraid because that's what healers do. (Gitana, healer)

Vested

With the exception of Lidia, every woman commented on the importance of buying-in to the spiritual work performed by the folk healer, and was determined by the code "vested/buy-in". All of the women in the sample shared the belief that their cancer had been influenced by metaphysical forces. All of the women also shared the belief that an investment of faith is required on the part of those using Santería healing services which is a defining feature that distinguishes it from conventional medical treatment use. The women felt confidence and conviction is needed for these spiritual interventions to 
be effective. "One has to want the help to be helped." Gitana clarified, "We all have to pray for it. This is not grab a chicken, pass it over me, I’m done." Oneida defended, "Yo lo hice todo con fe.” [“I did it all with faith.”]

\section{The Experience of Santería}

The Santería treatments and interventions received by the women varied and were captured by the code "experience of Santería”. Sirenita, Oneida, and Lidia described shells while Caridad described the opele being used to divine and determine the details of the cancer. Caridad also talked about buying her needed materials for the rituals, "I would go to the botánicas to purchase whatever they told me to purchase.” The rituals performed varied as well: Mariposa, Cristina, and Fatima focused on interactions and prayers with the orisha in their homes; Eddie and Oneida did a simple cleaning with an egg; Mariela attended spiritual sessions; Eddie, Madre de Agua, Elena, and Mariela participated in "rompimientos”; Gitana, Simone, Lidia, Andrea, Sirenita, Fatima, and Caridad had ebos performed which included animals. Regardless of the features, these rituals were all interactions with the metaphysical world in an attempt to treat the women's cancer.

All of the women in the sample described having positive thoughts and sentiments as they participated in the various Santería activities which mostly included a sense of hope and confidence in the effectiveness of the activity that would contribute to her survival. Thoughts and feelings experienced after the Santería activities were all positive as well and included a reduction in fear, anxiety, and stress. Oneida illustrated, "Y el tenía preparado un huevo, y muchas cositas así, y él me hizo una limpieza con aquello y 
yo fui de lo más serena, de lo más bien para el hospital.” [“And he had prepared an egg, and a bunch of little things like that, and he cleaned me with that and I went to the hospital feeling serene and quite well.”] The codes "thoughts/feelings during ritual” and "thoughts/feelings after ritual” captured this.

The Spiritual Entities of Santería

The women indicated varying levels of knowledge on the higher powers, or spiritual entities associated with Santería (i.e., orisha, santos, spirits, etc.), but all of the women interpreted these entities as being sanctioned and authorized by a supreme power to intervene in our lives. All of the women indicated feeling a personal closeness to these entities regardless of their understanding of them. Moreover, the women felt the spiritual entities also held an affinity towards them and were perceived as "parents" or "protectors". This created a sense of a direct and intimate relationship with these spiritual entities. In turn, a sense of being taken care of and defended against the cancer and potential negative outcomes of the illness was generated. This was captured with the codes "personal relationship::spiritual entities" and "trust::spiritual entities".

They're ever-present. I knew I wasn't gonna die. I knew it wasn’t something that was gonna kill me. I knew that they would not allow that to happen. They would not allow me to leave my children. (Eddie)

\section{Tangibility of Santería Folk Healing}

The eleven women who had used Santería spiritual work prior to this incidence of cancer had already experienced tangible results which reinforced their faith in its 
effectiveness. They reported results of other instances of health and illness having been altered via Santería services which produced a very specific sense of confidence in the current Santería treatment they sought and received for their cancer.

But then the first 28 days of my rayamiento my life changed. Everything I asked for, everything I asked for within a very short time period really happened with the exception of two things. And then those happened within a year. (Eddie)

\section{Doubt}

There were instances though in which desired results did not come to pass or the experience with Santería folk healing or the healer generated doubt as with Caridad and Gitana. However, based on the women's accounts, disappointing incidences were not sufficient to deter further consultation from folk healers and the use of spiritual work. In addition, Mariposa explained her overall struggle with being a pragmatic scientist and a faithful believer:

I guess I needed to hear that it would be alright and my true expectation from bajando la soperas [bringing down the containers] were that they were going to fix this. Because I know in the religion los santos [the saints] have made miracles. And so for me, I didn’t know if my miracle was I'm a stage one, you know, because I caught it early, so it's hard to be, it's very hard to be in the religion.... because I'm a scientist. And you straddle the hard proof, fact based on science versus the spiritual part where I believe in miracles and I believe in los santos [the saints], and mi angel de guardia [my guardian angel] and you know, so many things that could have happened to me in my life that my life was saved because I 
know that they love me and they protect me... But sometimes a part of me just doesn't believe. And so cuando baje la sopera [when I brought the container down] in the back of my head, the scientist in me was saying, 'You're wasting your time.’ You know?

Mariposa's quote offers clear insight into the tenacity of Latinas' acceptance of a metaphysical domain, Latinas' reliance on their spiritual beliefs, and the comfort spirituality offers Latinas. Despite doubts that may be had, this sample of Latinas indicated confidence in the Santería belief system, its practice, and its varied cast of entities. These women found soliciting this spiritual assistance in their efforts to overcome cancer to be helpful. Two separate codes contributed to this theme, which were “doubt” and "tangibility of Santería folk healing”.

Influence of Santería on the Cancer Experience

Metaphysical Influence

Among all of the Latinas sampled, there was a clear belief that there are metaphysical (supernatural) forces that influence and impact their health captured in the analysis by the codes "metaphysical influence”, "spiritual component”, and "healing one's spirit”. There was no question directly posed aimed at the capturing thoughts on metaphysical understanding since the literature indicated this is a commonly held notion among Latinos. The discussions were clearly predicated in this common Latino view of illness. All women in the sample were anchored in an understanding of a symbiosis that exists between the metaphysical and the physical worlds. The metaphysical, entities and their participation, were not apart, but embedded and a part in what is everyday life and 
experiences. All of the women's perception of their cancer was grounded in the notion of there being a metaphysical influence on their health status.

The illness...my health is getting worse for some reason, I need a hip replacement, my bones are weaker. Now I believe that this spirit is really like on top of me, you know? (Mafe)

\section{The Spiritual Component}

Given the foundational belief that the metaphysical was affecting their health status, all of the women in the sample perceived there was a spiritual component to the cancer. Women indicated healing "one’s spirit” was necessary, and that wellness could only come with balance which included attending to and treating the spiritual component of the cancer. They discussed that we are more than physical beings and are just as much energy beings with an essence, or spirit. That spiritual component of ourselves is aligned with our physicality to create our whole self. Therefore, what seemingly is "physiological" such as the cancer, also has a spiritual component associated with it which needs treatment. Folk healers were the ones identified as being best equipped to attend, bring balance to, and cure the spiritual component of the cancer. Those women who were healers themselves expressed a keen awareness that their role is to heal the spiritual component:

Doctors are trying just to cure you, to cure the illness you have with medications but they don't go through your spirit... because I think in life it is not only material things. I mean it’s not, it's not what you see...the body is composed by body and spirit, one person. (Mafe) 
...because your body is not just physical. It's body, mind, and spirit. And so from that perspective, from the spirit, your energetic part, your spirit is equivalent. And so you can bring the equilibrium or balance to that by bringing other modalities of healing. (Simone)

\section{The Cancer Experience}

Throughout the discussion that the interview generated, the experience of what it was like living through cancer was described and captured with the code "the illness experience”. The participants spoke about undergoing a range of emotions simultaneously as things progressed very quickly from diagnosis to post-recovery with minimal opportunity to process everything that was happening. The women illustrated the moment of diagnosis, surgery \& treatments, dealing with the overall physical rigors of the illness, and detailed the emotional and psychological strain which included affecting their sense of sexuality.

\section{Diagnosis}

The code "stressor::diagnosis" generated this section. The question posed regarding the moment of diagnosis produced consistently the same answer except for two participants: Mafe, who stated she was ok and accepted the news and Fatima, who stated she was prepared because the spiritual entities had already warned her. However, with the exception of Fatima, the remaining 14 participants shared in the same reaction when diagnosed which was the immediate thought of death. Being told they had cancer was compared to being given a death sentence and created stress and anxiety that were 
"inescapable". A few of the women termed the news as "traumatizing" and showed signs of emotion when they recounted that moment of diagnosis. The women illustrated a paralyzing fear that overwhelmed them to the point of emotional numbness and moments described as "checking out" and that life feels suspended. A few women defined it as a crisis and posed the question "why me". The women described feelings of panic which contributed to a sense of desperation. For those who had not used Santería services prior to the cancer diagnosis (Mariela, Elena, Mafe, and Simone), this desperation encouraged openness to non-conventional options such as Santería (which they had reservations about or had not considered using prior.) Madre de Agua stated, "siempre piensa uno que se va terminar todo.” [“One always thinks that all is going to end.”] Lidia explained, “Bueno psicológicamente tú piensas que cuando te dicen esa palabra de “cáncer” ya está tu final de tu vida.” ["Well psychologically you think that when they say that word "cancer" that it is the end of your life."]

Those who had children and grandchildren felt their younger generations still needed them and feared for them as well. Andrea and Mariela who were under 40 and had not yet had children feared their plans to have a family would never come to pass (along with other things they were striving towards.) Mariela shared, "De que todos los planes que tenía para mi vida, como, o sea, todo mi mundo vino hace abajo. Sea, todo lo que tenía planeado, tener una familia, tener hijos, todo se vino abajo.” [All the plans I had for my life, like, my whole world fell apart. All that I had planned, have a family, have children, it all fell apart.]

I have an autistic child that is always going to need mommy that's never going to have a 'normal' life. You know, she’s never going to grow up and marry and have 
children and be self-supportive. And yet, I have another child, you know, that needs me. (Gitana)

Fatima was the only participant who experienced a contrasting reaction due to what she describes as her spirituality in addition to guidance received via Santería services. She was confident that there was no threat of death and anticipated receiving news that she had an illness. "I knew I had something. I was prepared for it. I had dreamed it. I knew it. I was connected with my higher up. It’s like I had this tranquility.” Elena explained that even beyond successful treatment, the worry and stress of rediagnosis remains:

Porque eso tiene un periodo de regresión, no? De que tienes que estar en como remisión una persona como por cinco años. Entonces, yo pienso de que también con lo del cáncer es que experimente, o sea, que tuve todavía no se llegado todavía lo cinco años, que entonces eso también causa stress. [Because that has a time period that it can return, no? That a person has to be in remission for like five years. So, I think that with cancer, as I have experienced, I haven't yet arrived at the five years so that also causes stress.]

\section{Treatment}

Nine of the women underwent surgery as a treatment option, with a few of them requiring chemotherapy or radiation therapy thereafter. The women described the challenges that came with treatments, particularly the chemotherapy. They reported physical pain, nausea, exhaustion and an exacerbated sense of anxiety and nervousness. Recovery from surgery was taxing and for Caridad it required physical therapy to learn 
how to walk again. Time periods following chemo rounds were described as incredibly depleting and disorienting which interfered with the women's capacity to "keep up" and function. Mariposa recalls, "I really was in a fog for a number of weeks. I would say like a month.” The code "stressor::treatment” contributed to this section.

\section{Social Role}

The code "stressor::social role” captured a significant source of stress and anxiety, and a concept that emerged as an obstacle to the women's recovery which was their concern over maintaining their social role as caretaker within their family. Since the moment of diagnosis, the women's main concern was how the outcome of their cancer experience was going to affect those closest to them, mainly their children and grandchildren. All but the two young women who did not have children/grandchildren focused on 1) not disrupting their family’s routine by "keeping up" with day to day functioning as best they could and 2) actively protecting their family emotionally by appearing "strong” and as though nothing was wrong. A few women described the unacceptability for a Latina to show an inability or incapacity to manage herself and deal with the cancer. This concern over their family limited their ability to seek and receive support among those closest to them. One woman, Caridad, even verbalized her concern over her husband being left alone were she to pass away. She felt her intention to find him a wife that would be good to him and their children was expected of her and “unselfish”. Fatima described, “A woman’s nature is to take care of others and not worry about her wellbeing and unloading onto others.” 
Being Latina, we are raised to not have fear. Being Latina, at least my upraising as a Latina, has been, you know, ‘sale y comete el mundo' [go out there and take over the world]. You can't afford to tell somebody, you can’t afford to sit down and cry over your emotions. It just doesn’t happen. (Gitana)

\section{Sexuality}

Three of the women discussed their concern over their sense of femininity and sexuality which are highly valued female qualities in the Latino culture. They experienced anxiety over their partner's potential reaction to their body after treatment and its effect on her ability to remain attractive to him. They also indicate what resembles grieving over the loss of a part of themselves. Fatima explains, "My sexual life is going to change. How am I going to appear to my partner? Your life does change, emotionally, because of losing a part of your body, you know, a part that has been with you forever.”

Importance of Psychosocial Health during the Cancer Experience

Most of the participants expressed throughout points in the conversation the importance of psychosocial health during this experience with cancer and was coded as "psychosocial element::importance”. They discussed the necessity of maintaining a healthy “state of mind” since one’s psychosocial well-being had physiological implications that affected the cancer trajectory. The women believed strongly that thoughts, feelings, reactions, perceptions, level of optimism/pessimism, and whether one considers herself victim or victor significantly determines likelihood of survival. The women believed positive thoughts and mood strengthen the body's resilience against 
illness. Conversely, a negative disposition will burden the body and exacerbate its deterioration. The women asserted that it was a crucial component in beating cancer and that those battling the illness need to focus on maintaining a positive outlook. With much of the cancer being outside of one's control, managing one's thoughts and feelings is a piece of this experience over which the women can actually exercise some measure of control, in turn boosting their morale. Oneida explained, "Porque si tú te pones mal, lo dicen los médicos lo dicen, según el ánimo tuyo así trabaja la enfermedad. Estar siempre, 'Ahhh, que esto, ahhh, esto no se me vas a quitar nunca.' No, eso no es bueno.” ["Because if you are down, the doctors say it, the illness functions just like your mood. To always be, 'Ahhh, this, ahhh this is never going to go away.' No, that's not good.”]

Fatima made an interesting point about the needs of Latinas and the affirming psychosocial impact of being "heard”. She stressed an essential component to psychosocial well-being for a Latina is to have an outlet, an avenue allowing, permitting, and possibly encouraging her to express herself in a very authentic manner. She referenced formal mental healthcare as an option for psychosocial support. This reference was predicated in the belief that such support is only available externally because it is not available among Latinas' inner circle of family and closest friends due to the role Latinas are expected to maintain as caretaker.

La mujer lo que quiera es que le escuchen. They want to be heard. They want to be listened to. Hay mujeres que no lo pueden pagar (psicoterapia). El seguro no se lo cubre. Y eso es muy importante. Eso es algo sumamente importante por eso muchas terminan buscando ese suporte en otras áreas. [What a woman wants is to be heard. They want to be heard. They want to be listened to. There are women 
who cannot pay for it (psychotherapy). The insurance won’t cover it. And that is very important. That is something extremely important which is why many (women) end up seeking that support in other areas.] (Fatima)

Mafe and Caridad argued adamantly that mental and emotional well-being is vital since they struggled with overcoming stress and believed that it resulted in negative outcomes. They assert that the impact of the stress they underwent brought forth the cancer.

I think stress even gave me the cancer. I was passing through a difficult situation with the divorce, my sister was traveling to another country, another place, all the way to Washington State. My mom was sick. My father was diagnosed with cancer, terminal cancer, so he had only six months to live. And everything was together...So all of these negative things...I was paying all the bills and the stress of maintaining the house, paying the cars, all of these things, this stuff made me, I think it was something that made me have the cancer. (Mafe)

Psychosocial Effect of Santería Folk Healing on the Cancer Experience The process of dealing with cancer is strenuous, turbulent, and unpredictable. The experience with Santería and with Santería folk healers was reported as being positive for all women and coded with "psychosocial element::folk healing”. These spiritual interventions provided comfort and emotional/psychological relief during a very difficult time. In regards to the diagnosis, the Santeria spiritual work and consultation with the healer offered a spiritual understanding of the diagnosis such as possible sources and causes of the cancer. For nearly half of the participants (Mafe, Mariela, Elena, Gitana, 
Andrea, and Caridad) it answered their question, “Why did this happen to me?” More importantly, it offered them a resolution (i.e., spiritual work intended to cure the cancer, ensuring she'd receive the correct medical treatments, ensuring the treatments would be safe, ensuring successful outcomes to the medical treatments, etc.) Even prior to having a definitive medical outcome, the spiritual work completed offered an anticipated outcome. The trust placed in the healers and the spiritual entities reinforced the expectation that the anticipated outcome offered would come to pass, which created a great deal of hope among these participants.

The spiritual consultation and work offered the participants a blueprint on what to expect and how to proceed with handling the cancer experience. The participants reported that the guidance/information obtained through the consultations and spiritual work reduced their stress and anxiety levels significantly by giving them insight on the dynamics of what they were facing. What initially was an "unknown" trajectory of the cancer and how it would affect the participants and their families was in large part resolved with the consultation and spiritual work. This subsequently impacted the fear experienced by the women by reducing it and cultivated instead a more positive outlook. The participants described having been able to achieve a sense of calm and psychological/emotional relief that they were not able to gain via any other means. This alleviation of fear, stress, and anxiety positively affected the participants' quality of life by providing them an opportunity to return to what Mariela referred to as "normalcy" and respite from the depression she had been experiencing.

The logistics of the healer/client relationship and the effecting of the spiritual work itself also contributed to bettering the participants’ psychosocial health. The 
interaction shared between the participants and the healers and the process of completing the spiritual work was unique in that it allowed for the suspension of traditional Latina social roles ascribed to the participants. A distinct space was circumscribed for these Latina participants where they could be completely candid with their reactions to their cancer which was not encouraged in any other domain. The participants were not only allowed to face their authentic psychosocial response to the cancer but they were able to directly verbalize it to the healers and the spiritual entities via prayers. During the course of completing Santería spiritual work, the participants let out feelings, concerns, fears, and the weight of their reality, and were able to gain comfort, support, and reassurance. As mentioned previously in the section "Participants' Experience with Santería," the participants consistently reported a sense of confidence in the spiritual work being effective, a sense of their health being restored, and reassurance that the outcome would be one of triumphing over the cancer. Gitana, speaking as a healer, commented on her clients:

They leave with faith...Whether prayer is good for the soul, or not, once you finish your session, you see hope. They walk differently. They hold their heads up differently. You see a glint in their eyes that wasn't there 40 minutes before, an hour before.

However, Gitana as a patient and client of another healer, in contrast, had not had as positive a psychological and emotional experience when having spiritual work done to deal with her cancer. She was not getting the results she sought so every subsequent ritual made her progressively anxious and upset because it was not producing the successful 
outcome desired (eliminating the cancer). In place of hope, perceptions about the inability to "beat" the cancer and feelings of defeat were instead exacerbated.

\section{Cured of Cancer}

Even though the focus of this study was to explore the psychosocial influence of Santería on women with cancer, the participants shared the positive outcomes of Santería spiritual interventions performed which were coded as "cured”. Madre de Agua, Eddie, and Sirenita assert tumors that had been detected could not be found upon return to the doctors/hospital. The women affirm the tumors literally disappeared. Mariela had a month of numerous trips to the emergency room in which she could not be diagnosed correctly. It was not until the evening following her rompimiento ceremony in the ocean that she received the accurate diagnosis of gallbladder cancer and was operated on within a few hours. These women credit the spiritual work performed as curing them from the cancer and saving their lives.

So then the doctor comes in and he puts up the pictures of the mammogram. And he's like, 'This is your breast here. This is the area we were looking at. There's the mass. There’s your gland.' He’s like, 'This is your mammogram because it came with the left breast that we have definitely found the scar tissue from your previous lumpectomy where we removed the lump from that breast. So we know that this is your mammogram. Ok, this is your new mammogram.' He’s like, 'And that's why I had her do a mammogram on the left breast just to make sure that it was the same one. And there's your scar tissue from the left breast and here's your right breast.' I was like, 'Ok.' And there’s nothing there. 'Ok, so what were 
you looking for?' 'We were looking for this. And we were trying to measure that so we could measure density, size, closeness, everything. We can get a better picture, and it’s not there.' (Eddie)

Mariposa in contrast did not feel any spiritual work would make her polyps disappear. She felt the religion (Santería) and interacting with the orisha would help on a certain level but not necessarily eliminate the need for surgery:

I didn’t think that La Religion was going to cure my cancer. They were not, I really felt in my heart after I saw that grape sized polyp that La Religion wasn’t going to make that shrink and go away, make those cancer cells disappear.

Participants’ Experience with Conventional Healthcare and Mental Healthcare It was determined that the general question used in the first version of the interview guide "What was different about this (folk healing) than going to see your doctors, a therapist, a support group with other women living with the experience of cancer, etc.?” needed to be further broken down into three parts to capture these distinct areas of formal care and mental health care (doctors versus therapists versus support groups.) This theme was built by a number of codes: “comparison to medical doctors” “comparison to mental health”, "psych/therapist::experience”, “psych/therapist::perception”, “support group::experience”, and “support group::perception”. Regarding therapeutic services received by Social Workers, Psychologists, or Psychiatrists, I coded and included in the analysis any therapy services received beyond those having to do with her cancer. It was an additional effort to better understand Latinas' underutilization of formal mental healthcare options. Those who had 
never seen a Social Worker, Psychologist, or Psychiatrist were asked their perception of what it might be like to receive such services when dealing with the cancer. This question on perception was also asked to those who had never attended a support group for Latinas with cancer.

\section{Comparison to Medical Doctors}

Even though the research questions focus on mental health, the women were always inclined to refer to their experience with medical doctors in comparison to Santería folk healers. The value the participants placed on the quality of the relationship shared with their doctors (and with the folk healers) was critically important to them and apparent. None of the participants expressed any medical mistrust in the physicians or the treatments. Most of them however, (Eddie, Gitana, Simone, Mafe, Caridad, Sirenita, Mariposa, Mariela, Elena, and Fatima) indicated varying degrees of dissatisfaction with their patient care experience. The women interpreted the nature of the interactions with the medical doctors to be uncaring and unconcerned. Participants perceived visits to be rushed with the doctors' disposition being one of detachment. This created for the women a sense of their physicians having a lack of interest in them and perceived minimal effort on the part of the physicians to ease the experience. They speculated the lack of interest being possibly rooted in the doctors being materially and compensation driven, lacking time, lacking compassion out of professional necessity to be able to perform their job function, or plainly not caring enough. Among the participants, this promoted feelings of loneliness. The participants also described a fear that the physicians were not necessarily 
interested in the outcome of their cancer which would affect the physician's level of determination and commitment to ensuring their survival.

With the exception of Mariposa, these participants also believed their physicians were unapproachable. They did not feel their questions were welcomed. They avoided asking for clarification because the doctors appeared annoyed. This perception of unapproachability and its dissuading effect on the participants' entitlement to ask questions and engage in conversations over their care and treatment exacerbated a lack of health literacy among the women. The participants' concerns for the most part remained unaddressed and they had to pursue other avenues (family and friends, healers, the internet, etc.) to obtain information on the cancer, treatment, and matters related to the cancer experience. This lack of interest and unapproachability eliminated any opportunity for the participants to adequately process and pose candid questions and concerns in reaction to the information they'd receive in what they described as hurried impersonal interactions with their oncologists.

Con el médico, “Si, hola. Llegue. Ciao. Ya te vi. Son \$500.”... no tengo nada en contra de los médicos. Son unos profesionales. Hay unos muy buenos pero hoy, por hoy son cinco minutos de consulta y te cobran un ojo de la cara y parte del otro también. [With the doctor, "Yes, hello. I’m here. I saw you. That'll be $\$ 500 . ” . .$. I have nothing against the doctors. They are professionals. There are some very good ones, but today, today it’s a five minute consultation and they charge you an arm and a leg.] (Sirenita)

As mentioned previously, none of the participants expressed concerns regarding the capacities of the medical doctors. The physician's ability to heal was believed to have 
come from their educational preparation and from advanced technology that accompanies the medical sciences. Participants however, also attributed doctors' ability to heal to having a spiritual gift, or “don”, similar to that possessed by the folk healers. Participants also explained that the same metaphysical influences that grant the folk healers' healing capacity (spirits of the dead, orisha or santos, God, and the Universe) intervene and guide the doctor's capacities to heal as well the doctors' judgment and the medical and scientific treatments employed. Eddie described a conversation she had with her Godmother, “We're going to make sure that it’s Zarabanda's hand that's holding that knife at the end of the day; that it's not that doctor alone; that somebody's always going to be with him.” (Eddie)

This was an area that was expanded upon when supplemental questions were added to the interview guide based on data that emerged from the earliest interviews. Codes that contributed to this theme were "medical doctors::lack of interest", "medical doctors::unapproachable”, "comparison with doctor/medical procedures/formal services”, “experience with oncology”, “ability to heal::doctor”.

Comparison to Formal Mental Healthcare

Therapists (Social Workers, Psychologists, Psychiatrists) - Experience

Five of the participants, Simone, Mariela, Mariposa, Elena, and Gitana, had undergone therapy sessions with formal mental health providers and Madre de Agua had experienced interactions with formal mental healthcare providers when her daughter was receiving services. Simone, Mariela, and Elena had positive sentiments towards the providers and the services explaining how it can help with psychological processing and 
emotions. However, they felt the services were still lacking and did not provide the psychological and emotional relief that the folk healing offered. As much as they liked their therapist, it was still a more formal relationship and lacked the dynamic and interaction the participants were able to share with their folk healers. The participants also stressed again that a large part of what contributed to the psychosocial reprieve they sought was the spiritual element of the folk healing and its ability to resolve the situation. Again, this is predicated on the participants' firm belief that there is metaphysical influence, if not cause, to their cancer. Answers were given, spiritual work was completed to ensure the participants' survival, and an anticipated outcome came to pass in a short amount of time with the Santería services. The formal mental healthcare services did not offer the same process, outcome, and definitely not a resolution since they did not attend to the spiritual component of the issue for which services were sought. They indicated again that for a resolution to be achieved psychologically and emotionally (as well as physically) the spiritual element of the cancer needed to be attended to.

The therapist? It's a different dimension of a tool. It's not spiritual. It's psychological... So you have the mind, and that's where the therapist comes in. But there's nothing that's really there targeting your spirit. And so, and those are two very important components, mind and spirit. And there's very few psychologists that will do both. And so to tap into the energy of your spirit, to bring everything in alignment, so that everything is so that there's some cohesiveness. (Simone)

Madre de Agua however, commented that she did feel therapists also possessed a healing "gift" since mental health symptoms are less tangible and more difficult to 
diagnose and treat. Regarding the other two women who received services, Mariposa voiced disappointment in the experience and Gitana felt mental health providers are just as disinterested and uninvolved as medical doctors. She stated that the therapists are more concerned with the systemic protocol, such as how many sessions and how much time for each session will be paid for by the insurance, instead of being able to provide quality care that is built on a more fluid relationship.

\section{Therapists (Social Workers, Psychologists, Psychiatrists) - Perceptions}

Four participants, Madre de Agua, Oneida, Fatima, and Cristina had not visited a therapist but shared that it could have benefits. However, Oneida, Andrea, Eddie and Mafe expressed a preference in seeing the Santería folk healer over a mental health professional. Once again it was anchored in the lack of a spiritual component in the formal mental health services. The perceptions by those who had not received these mental health services seemed to share the same expectations as those who had received them. They wanted to feel better and gain relief from their stress by means of having the source of the stress eradicated which was the threat on their life. The participants believed the Santería services would either cure them or ensure safe and successful treatment. Therapists cannot offer the same outcome. Fatima described the parameters of support that therapists/therapy can offer. She was explaining that even though therapy is beneficial, it is limited because it does not offer the room to change the situation and alter the course of the cancer, or one's psychosocial reaction to the cancer.

The psychotherapist will base his therapy on knowledge, psychological knowledge, psychology knowledge. The psychotherapist can help you to 
understand what you're going through, educate you, and the pluses and the minuses. Basically in the psychotherapy way you got to accept, and when you accept then you have to deal with it. (Fatima)

Support Groups - Experience

Four participants, Caridad, Simone, Elena, and Sirenita, had attended support groups for women with cancer, or support group functions. Sirenita accompanied a friend with stomach cancer and maintained such an adverse opinion on the groups that it was not an option for her when she received her diagnosis a couple of years later. All four women felt and described these groups as lacking a focus on positivity and instead nurturing a victim mentality. They accepted the support group setting as an appropriate venue for opening up and expressing their psychological and emotional distress. However, they did not find it promoted a positive outlook but instead exacerbated a negative frame of mind. Additionally, Caridad felt judged.

The support group that I went to was in South Miami hospital and I felt very judged. And I didn’t really feel that it was helping me at all...There were so many opinions on what I was doing. And they were "wrong". They said to me they were “wrong”. It was wrong for me to do that. So I kinda like, I went for a time and then stopped going. (Caridad)

Lidia at one time worked in a behavioral health center and participated in cofacilitating groups. She compared support groups to specific prayer groups done within the Santería community with the main distinction being that of spirituality. Lidia had never attended a support group herself during her cancer experience because she was 
another participant who considered herself "strong". However, she felt support groups were beneficial but like all of the other participants favored a modality that incorporated a spiritual component.

\section{Support Groups - Perceptions}

For those who had never attended a cancer support group for Latinas, perceptions were both positive and negative. Although Gitana had a very strong reaction to this idea of a support group as is evident in her quote, there is a shared element found in Mariposa's more subdued comment as well. Both women did not want to bear the emotional burden of other women's cancer experience and instead preferred to deal with their own cancer experience by themselves.

Because I am therapist myself, and in the mental health field, I didn’t want to sit in a room with other women. I didn't have the energy to be supportive for them. I'm so sorry to say that. It's my healing, it's always very internal. I'm sorting everything out in myself. So I can't, I don't want to be around people. I can't be nice to you. I don’t even want to talk to you. (Mariposa)

Cristina made comments indicating that supports groups can traumatize you, but on the other hand, the sharing among women in similar circumstances with a trained facilitator can be helpful. Oneida, Mariela, Mafe, and Fatima also considered the shared experiences as beneficial, but did not have a need to attend one. Eddie and Andrea both felt the same way towards support groups as they did with therapists which was not necessarily positive or negative, but instead held a simple preference for Santería over 
any formal mental healthcare options because of the resolution they believed was offered by the spiritual component provided by folk healing. 


\section{CHAPTER 5}

\section{Discussion}

This study stands apart from other studies on the role of spirituality and employment of religious coping among Latinos which generally focus on the areas of faith, prayer, and church involvement. Quite regularly, statements about Latinos’ use of folk healers are made in these studies, but rarely does its mention extend beyond a generic and non-descript comment classifying folk healers as a rote Latino health practice appendage. There has been limited effort in distinguishing the mechanisms underlying the motivations of Latinos' use of this particular modality as it relates to their healthseeking behavior thus creating a challenge in contextualizing the data and results in a study such as this. Kleinman’s Internal Structure of Local Health Care System within his Explanatory Model of Health and Illness helps us understand this area of commonly sought health care modalities identified as the popular sector. It helps delineate the attributes of professional or folk services that become branded and positioned in the popular sector and is one of the few pieces of literature that helps reveal how and why these attributes constitute “cultural congruence”.

This sample illustrated the folk healing modality of Santería as a commonly sought healthcare option and thus, does in fact exist in the popular sector of services accessed by Latinas with cancer. This sample also illustrated the function of the popular sector which housed not only the utilization of Santería, but that of professional oncology medical services as well. This challenges Gurung's argument that Latinos exclusively use professional medicine or folk healing (2013). The sample in this study (all women had been in the U.S. for over 10 years; nine participant's household income was over $\$ 40,000$ 
with four of participants’ households earning between $\$ 90,000$ and $\$ 120,000$; five hold AA degrees, four hold BAs, and one has a $\mathrm{PhD}$ ) also challenged the commonly held belief reinforced by the literature, that the Latino cohort using folk healing, specifically curanderos, tends to be those of lower SES. Even though Santería is historically a Cuban tradition, the composition of this study sample (4 out of 15 women of non-Cuban extraction) is shaping the argument that a Santería diaspora has been, or at the very least is in the process of being, established.

Specific Aims \& Research Questions

Aim One set out to determine the attitudes of Latinas living with cancer towards Santería as an informal mental health support that assists in coping with the psychosocial sequelae of living with cancer. Latinas did not pursue Santería services as a form of psychosocial support. They sought Santería services as a treatment to the cancer and its physical symptoms. In doing so though, they inadvertently gained psychosocial support from the experience.

Aim Two set out to explore whether Santería offers Latinas effective mental health support that assists in coping with the psychosocial sequelae of living with cancer and if so, how? As determined among this sample, yes, Santería offered these Latinas effective mental health support and assisted in coping. How it did so is addressed by the three research questions.

Question one asked what effect does Santería have on the psychosocial sequelae of Latinas with cancer, and question two set out to identify the mechanisms of the effect. Santería had a positive effect on the psychosocial sequelae of this sample. The 
mechanisms creating this positive effect were the confidence, hope, and alleviation of stress and anxiety the Santería experience granted. The women had an enormous amount of confidence in the healers' and spiritual entities' abilities to heal them from the cancer. The participants were confident they would emerge as survivors from the cancer experience which afforded them a great deal of hope that their health would be restored and in turn, alleviated a great deal of their stress and anxiety. Santería also created an appropriate space for the women to be open and reveal their genuine reactions and concerns about their cancer which they were not able to do elsewhere.

Question three set out to determine the characteristics of Santería that distinguish it from formal mental health services (psychiatrists, psychologists, therapists, counselors, and support groups). The characteristics that distinguish Santería from formal mental health are 1) the spiritual component of healing that is addressed, 2) the personal relationship shared with the healer, 3) family and/or close friend facilitating and supporting the use of Santería, and 4) empowerment. One through three are characteristics are identified in the literature as Latino cultural values.

Spirituality is a cultural cornerstone and the religious coping relied upon is impervious to evidence. This was exemplified in Caridad and Gitana who relentlessly sought spiritual intervention despite conflicting and unsatisfactory results. Using Santería services, the women felt they were being healed at the source which is the spiritual component of the illness to which medical doctors have no access. In addition, the personal relationship with the folk healer, whom is credible and trusted, offered a sense of incomparable support whereby the healer shared in the cancer reality by being on the front line with the women, ultimately “championing” them. If the healer wasn't a 
"conocido", or known to her before, the healer became so almost immediately. Latino families exerted a substantial influence over treatment options, hence contributed to Latinos' underutilization of formal mental health and the continued use of folk healers by younger acculturated generations.

Empowerment, however, directly opposes the fatalism and machismo that are two other identified Latino cultural traits in the literature. The practice of Santería as a Latino institution is not without its share of built-in patriarchy, yet the participants were able to experience momentary relief from the inherent gender roles when they were receiving services as a client. Even though family was a significant source of support for Latinas, there was always the role of nurturer and caregiver to maintain which neutralized any capacity for the family to genuinely function as a source of support. Santería provided a "safespace" for the women where they were liberated from such roles and from what they could or could not say, think, feel, etc. regarding her cancer. This milieu Santería provided allowed for full-disclosure where the women could be completely honest, unload the weight of her stress/fears/concerns, and feel heard.

These participants also demonstrated tenacity, and their use of Santería challenged the accepted Latino trait of fatalistic resignation. This sample exhibited a determination to overcome the cancer. These women demonstrated a fighting spirit identified by the code "determined resiliency". Even where acceptance was indicated, it did not suggest defeat. This exertion and employment of accessible treatments beyond conventional oncology combined with the opportunity to worry solely about her and her privations, created a sense of empowerment for Latinas. These women had no intention on consenting to "defeat" or the negative effects of their cancer experience on them or 
their loved ones. As Gitana explained, "You look for any and every option when it comes to facing a life-threatening situation like cancer.” Santería as an available treatment option enhanced the participants' internal locus of control by offering them a sense of control over their situation.

Figure 2- Santería Characteristics Contributing to Psychosocial Well-Being

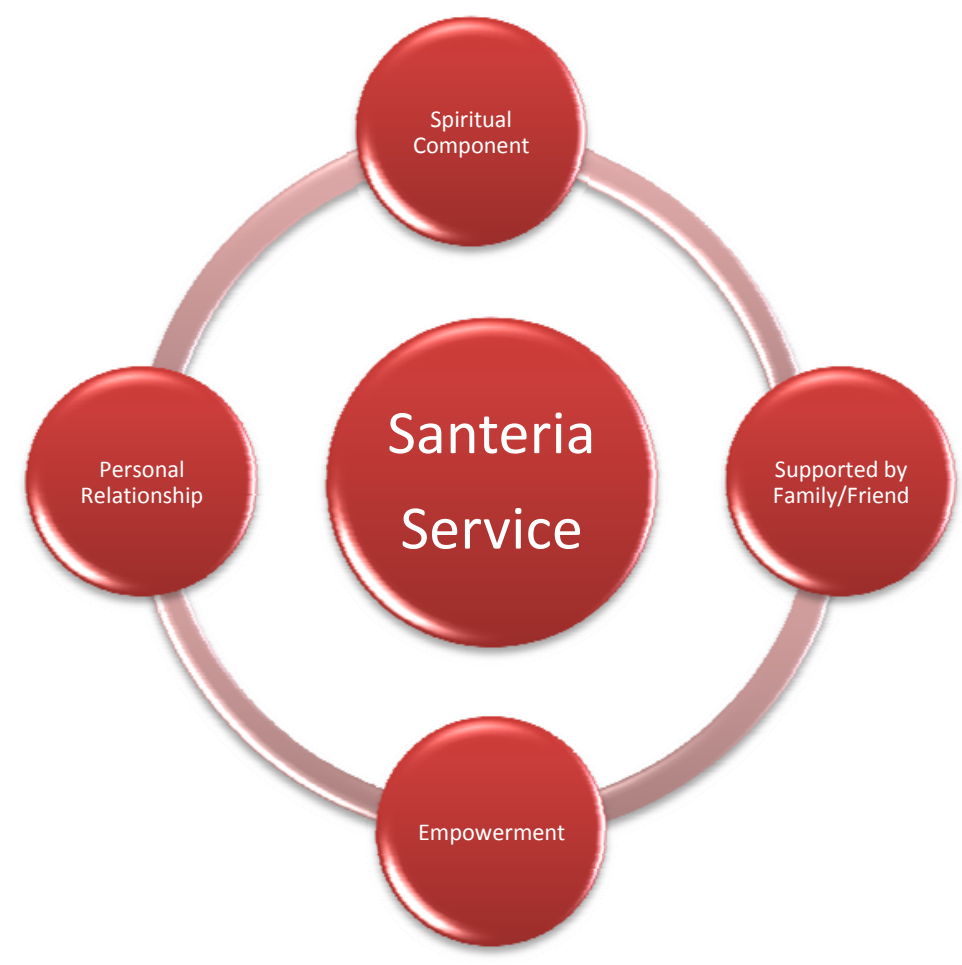

\section{Focus Group}

The findings from the interviews were presented to a focus group that was comprised of Mariposa, Caridad, and Mariela. Simone and Cristina were also invited to participate to ensure all demographic categories would be represented in the focus group. 
Cristina is an older lady who was not feeling well the week prior when contacted and declined to participate. Madre de Agua, who shared the same demographic characteristics, was invited in Cristina's stead, but declined because she was seeing clients the morning of the group. Simone stated she would try to make the group but could not confirm because she had already made arrangements to attend a work-related seminar the same morning.

The three group participants were in agreement with the all of the themes presented. A discussion regarding “empowerment” dominated. The obvious contributor to feelings of empowerment was the sense of control gained by actively seeking and utilizing Santería services while in a situation that made them feel powerless. They felt they were at the mercy of a healthcare system in which treatments were determined with minimal input on her part and instead by “uncaring” medical staff and insurance coverage. The women explained however, that there was a more foundational issue that contributed to this sense of empowerment which was one of expectations. Santería satisfied expectations since it is built on meaningful Latino values and offered these Latinas a unique component of empowerment that was otherwise unavailable. The group agreed that a Latina's self-denial of an emotional outlet is truly embedded in the culture. They explained how a Latina is not to burden her family therefore she cannot lean on them for emotional support. Additionally, she is expected to attend to her family's needs and absorb their reliance on her regardless of being ill or recovering. It was shared with the group that there was not one comment or insinuation made by the participants during the interviews about needing, expecting, or even feeling a desire to be cared for or sympathized over. A point was also shared with the group that the illness was described 
by a few participants in terms of a war or battle against the cancer. The group corroborated that psychologically this is how she views herself in relation to the illness which is that it is a war and cancer is the enemy. The group also insisted that there is indeed an irrefutable proclivity to protect those they love, the kids and older parents alike, from seeing them suffer the effects of this "war". So family being a major source of strength for Latinas also contributed to an exacerbation of stress and acted as an obstacle to psychosocial well-being.

When the group's conversation shifted to comparing Santería to formal mental healthcare services, the women explained that it's this same issue of expectation. The tendency to give support instead of receive it extends itself into the utilization of formal mental healthcare. The discussion indicated that support groups and therapy sessions cannot seem to successfully strike a balance between the formal models employed and this Latina feature of “unloading” one’s baggage onto others as unacceptable. Formal mental healthcare services are rendered ineffective since they are not equipped to accommodate this Latina attribute, in addition to lacking a spiritual component which is necessary to end the cause of the problem (cancer) which would result in true psychological and emotional relief.

The focus group also articulated that much of the hope and confidence Santería instilled was generated by the answers that it provided which distinguishes it from a) formal mental health professionals who cannot assure their clients that "things will be ok" and b) other spiritual interventions (i.e., consultations with ministers, priests, prayer groups, church attendance, etc.) which also cannot certify any particular outcome. The group also shared that the rituals serve a critical function and contribute positively since 
they are the embodiment of the cultural history of the tradition and what retains the tradition as meaningful. The women pointed out they felt it was more of a cultural connection rather than a religion ascribed to; an interesting distinction since all but the healers’ reported religious affiliation with a dominant religion, mostly Catholic and Christian. The personal relationship with the healer was validated as being fundamental since it is something that cannot be accomplished with conventional medical doctors.

Interview with Oba Ernesto Pichardo

On February 15, 2013, I had the good fortune of meeting with Ernesto Pichardo, a recognized and well-respected leader in the South Florida Lukumi community. He is the head of the Church of the Lukumi Babalu-Aye in Hialeah, FL which argued a case before the Supreme Court in 1992 for freedom of religious practice. This discussion was held before any interviews had been completed and much of what Pichardo explained validates the findings.

Pichardo began the conversation describing a framework for the belief system and the process of divination. He commented that we are all connected and sustained by a field of energy, hence the interaction between the metaphysical and our physical world. This illustrates the women's understanding of the metaphysical world influencing not only health but all parts of one's life. He explained clients find their way to healers via trusted family or friends. Pichardo continued to detail how clients arrive with expectations, seeking an explanation and a feeling of "safety”. Having received a cancer diagnosis creates a lack of safety and security, and the women seek the healer to understand the "why" and "how" of the illness and what could be done to mitigate it, thus 
restoring the sense of safety. He described how much of the client's belief does not necessarily rest in faith but on conviction derived from the validation, or "deliverance” as experienced by either the women herself, or by the trusted family/friend that referred her. It is the tangibility of the results that once seen, one believes and trusts.

\section{Social Work Practice Implications}

The social work profession is committed to meeting the needs of the most vulnerable and oppressed members of society which is particularly important in the context of cancer given there are significant disparities in various minority groups, including Latinos. Since the early days of the profession, social workers have held a crucial role in providing the earliest notions of culturally sensitive care within the U.S.'s highly eurocentric healthcare system that discriminated against minorities (Cabot, 1909). Social work continues to hold the responsibility of alleviating the underutilization that exists in Latinos’ access to culturally congruent psychosocial oncology care. Findings from this study can help inform micro, meso, and macro level social work practice.

\section{Micro Level - Service Delivery}

Before any discussion about models or therapeutic approaches is to be had, we must unmistakably understand the Latino value of personalism as foundational. These interviews have made clear how distinctly these patients recall "how" they were treated by doctors, therapists, and healers. The level of concern and support, and overall bedside manner, was crucial and defined much of the healthcare experience. 
In regards to therapeutic models, social work has long acknowledged the importance of spirituality with its holistic focus on person-in environment (Smith, 1998). It is no coincidence that social workers in end-of-life and palliative care were among the first in the profession to explore the significance of religion and spirituality as a means of relieving psychological distress. Mental health social workers were second to implement models of practice inclusive of spiritual elements (Smith, 1998). Since social work is such a broadly based profession in which all social workers will likely encounter clients facing life-limiting illnesses such as cancer, expertise in culturally congruent palliative care will weigh heavily on their capacity to intervene appropriately (National Association of Social Workers [NASW], 2004). As per the NASW guidelines (2004), a significant component of effective palliative care includes integrating the psychological and spiritual aspects of patient care so to offer "the prevention and relief of suffering by means of early identification and comprehensive assessment and treatment” (p. 10). Incorporating a client-centered service delivery model would afford clinicians the ability to provide such care and offer therapeutic approaches with flexible parameters that can be modified to include each client's cultural concepts, values, and belief system.

As Baez and Hernandez (2001) indicate, traditional Latino spiritual and healing systems have long been noted as mental health support. Nearly 30 years ago, it was noted that folk healers could be included in formal mental health treatment networks or teams providing services to Latinos (Schwartz, 1985). Therefore, a client centered therapeutic approach inclusive of spirituality and cultural elements have been shown to be effective (Baez \& Hernandez, 2001). In a systematic review conducted by this researcher on psychosocial interventions for women with breast cancer, only two of the 15 studies, 
Chan et al. (2006) and McKiernan, Steggles, Guerin, and Carr (2010) incorporated cultural relativity into the intervention with Chan and colleagues (2006) having reported robust effects. Also, Martinez and collegues’ (2008) Camp Alegria that was culturallytailored for its Latina oncology participants indicated success.

Taking from the participants’ experiences with their doctors once again, there was a clear disinterest, if not antagonistic reaction, from the medical professional upon learning of the woman's use of a folk healer. There was no attention granted to the participant's use of Santería as a treatment modality, her interest in it, or its relevance to her conventional medical treatment. This creates an atmosphere which further prevents patients from having a say in their own treatment options. The Latinas in this study signaled a desire to actively participate in their own treatment and betterment. The current therapeutic models employed by social workers still limit the amount of active participation on the part of the client. Cognitive Behavior Therapy is perhaps the mental health model demonstrating qualities similar to the Santería folk healing process and experience, but would still require accommodating the other relevant cultural variables. Traditional roles for women as nurturers need to be considered and accommodated to create that necessary space for Latinas to unburden themselves and strive for attaining well-being. Counseling and intervention models are needed which incorporate an approach that allows Latinas to assert themselves and fully disclose their use of all other informal and formal treatments that they are incorporating in their care, i.e., folk healers. It is important to allow clients to express what is of value to them and extend that into their mental health care. 
Meso Level - The Community

Much of the informal support Latinas with cancer have been receiving is from a natural support system (family, friends, the community, etc.) and through the informal networking accomplished there. Delgado’s work illustrates the stable position folk healers have held in Latinos’ natural support system and validates the valuable function of folk healers as a natural support in the lives of Latinas with cancer and their psychooncological needs (2007). The inclusion of these community elements (healers, churches, organizations, programs, etc.) that positively influence Latina clients should be brought into the fold of their treatment plan. Latinos would benefit from using strength-based models that incorporate natural supports into formal treatment teams. Additionally, cultural congruence and staff development trainings can include consultations on natural support systems or with folk healers themselves as Delgado and Humm-Delgado recommended from their extensive work in this area years back (1982).

\section{Macro Level - Policy}

Cultural congruence efforts began as a guide for interpersonal interaction between provider and patient, and later expanded to consider health systems. Demands for culturally congruent health and mental health services grew out of the failure of service delivery systems to be responsive to all segments of the population (Chin, 2000). The federal response was the development of the Culturally and Linguistically Appropriate Services, or National CLAS Standards by the U.S. Department of Health and Human Services’ (DHHS) Office of Minority Health (OMH) to provide a common understanding and consistent definition of culturally and linguistically appropriate healthcare services 
(Anderson, Scrimshaw, Fullilove, Fielding, \& Normand, 2003). These standards were set forth to respond to the need to ensure that all people entering the healthcare system receive equitable and effective treatment in a culturally and linguistically appropriate manner (Hoffman, 2011).

In an attempt to promote cultural congruence, the American Psychological Association's DSM-V and the World Health Organization's ICD-10 call for additional awareness on culture-bound syndromes further necessitating knowledge of cultural beliefs, values and practices (Quinlan, 2011). Otherwise, practitioners can easily fall prey to errors of diagnosis, inappropriate care, and poor compliance (Bhui et al., 2007). Therefore, Schim and Doorenbos (2010) recommend an expansion of thinking about cultural variables beyond strictly race and ethnicity, instead approaching it as a "sociallyconstructed and transmitted constellation consisting of such things as practices, competencies, ideas, schemas, symbols, values, norms, institutions, goals, constitutive rules, artifacts and modifications of the personal environment” (Fiske, 2002, p. 85). By advocating and lobbying for federal and local policies with an expanded scope of what is considered culturally appropriate, programs funded from such public resources will be obliged to diagnose minority patients, particularly in regard to culture bound syndromes, from a more flexible perspective.

\section{Study Limitations}

The study had several limitations, partly due to practical concerns. First, the study employed a non-probability sampling technique to recruit participants. Well-designed research conducted with rigorous methods contributed to the credibility and internal 
validity of the study using non-probability sampling techniques (Bernard, 2006).

Specifically, validity in exploratory research refers to the "whether the researcher can gain an accurate or true impression of the group, process, or activity under study,” and if so how? (Stebbins, 2001, p. 48). The study design incorporated a number of components to strengthen validity: (1) collected data from multiple recruitment sites and techniques (i.e., botánicas, chain referral sampling), (2) used well-articulated qualitative analysis techniques (i.e. coding procedure and constant comparison method), (3) employed maximum variation in the sample, (4) triangulated findings (a. incorporated a member checking stage for validity and b. personal communication with a well-respected, reputable member of the Santería community), and (5) researcher’s awareness of personal biases. The methods used were significant to validate the findings and for gathering information on which to base future work.

Second, sensitive issues such as the Latinas' experience of living with cancer, what they felt constituted effective mental health care for their psychosocial sequelae needs, and their regard towards Santería healers were not verifiable through any means other than self-report. The extensive training of the interviewer allowed for rapport building that successfully elicited accurate responses. Additionally, one participant doubted the confidentiality promised. Her concerns were addressed with constant reassurances of the confidentiality binding the research and the information's relevance and contribution to the study. Fortunately, she completed the study but did not refer her two goddaughters for recruitment.

Third, concern over translating the English interview guide to Spanish was resolved be using the translation back translation method. One individual translated the 
interview guide into Spanish, and a second individual translated the material back to the source language, English (Sperber, 2004). The independent back translator was blinded to the original English questionnaire therefore increasing content accuracy.

Finally, the sample size achieved was less than proposed. The final sample size of 15 , however small, was determined by the level of theme saturation achieved. As few as 12 interviews have been found to be sufficient to determine data saturation in qualitative HIV/AIDS social research (Guest, Bunce, \& Johnson, 2006). Analysis indicated by the ninth interview that articulable concepts and themes had emerged and were supported by multiple respondents in different interviews. No new themes emerged beyond the $12^{\text {th }}$ interview. Despite the small sample, incorporating a negative case was achieved with Simone who only used complementary and alternative medicine physicians (versus conventional oncology physicians) and did not agree with certain characteristics of Santería despite utilizing it and intending to do so again.

\section{Future Research}

This study afforded a critical appreciation for the procedures that are vital when working with the Latino community. As this study has plainly indicated, personalismo is one of the most fundamental values held by Latinos and a model of interaction that must be employed when conducting research with this group. Resources must be invested that allow for the nurturing of rapport with the community, primarily a considerable amount of time for relationships to be established. Much of successful recruitment and retention of Latinos are contingent on the networking established by the research team within the community, which will obligate time (De La Rosa et al., 2012). Work done in the area of 
folk healing will benefit from using a Community-Based Participatory Research (CBPR) model since folk healing traditions remain a somewhat intimate component of Latino culture that is very personal and subsequently well-guarded.

Future research on the use of Santería folk healers in the Latino population as it relates to public health, healthcare, and mental healthcare should begin by using larger samples and determining its relevance beyond the Cuban-American community. More exploratory work is needed to uncover Latinos’ reluctance to tell medical and professionals about their use of Santería folk healers, and also uncover the medical and mental health professionals' reluctance to regard their patients' and clients' use of such services. To do so, the perceptions held on the part of the patient/client and medical and mental health professionals' towards Santería folk healing use need to be articulated. In regards to measuring effectiveness: 1 ) development of $a$ ) client-centered models, $b$ ) strength-based/asset models, and c) models with a folk healer/spiritual healer component; 2) embedding resource information and promoting health, healthcare and mental healthcare services, support, etc. within natural supports and community networks (i.e., botánicas, folk or traditional healers, etc.); and 3) system or policy research on expanding the scope of culturally and linguistically appropriate services. 


\section{CHAPTER 6}

\section{Conclusion}

This study explored Santería’s function as a culturally congruent, ethnic specific form of psychosocial support among Latinas with cancer. Folk traditions are built on and embody their respective culture’s core principles and traits, and practices such as Santería serve as culturally relevant treatment options in addition to being belief systems. One such fundamental tenant of Santería is the belief that the metaphysical (supernatural) and physical worlds are intertwined, and that health and illness have spiritual implications. Even though Santería originated in Cuba and has historically been a tradition assigned to Cubans, such fundamental tenants are shared by most Latinos allowing for Santería’s practice to extend to all Latino subgroups as a material expression of shared values.

Santería is however a tradition that operates privately, but the tangibility of its utilization is evident with the presence of botánicas in Latino communities. The CDC's National Health Interview Survey acknowledges folk healers and the folk practices supported by botánicas as Complementary and Alternative Medicine modalities and has reported high use among Latinos. It is of the utmost importance that such modalities are granted attention as suitable options of mental health support for Latinos since this study supports the literature's discussion on Latinos' underutilization of formal, conventional mental health care services.

Aside from lack of access issues which is beyond the scope of this study, there is an inadequacy of culturally congruent conventional mental health support for Latinas. The pressures Latinas face, particularly regarding familial and social roles, create limited culturally appropriate psychosocial support options for Latinas. Latinas remain 
dissatisfied with conventional mental health care because such services do not take into account Latinas' unique reality and their commitment to the culture’s value system. This study identified acceptable versus unacceptable expressions of their illness experience among the Latinas sampled, and the elements that create the cultural incongruence with conventional mental health care were uncovered. Latinas’ inclination to avoid seeking mental health support coupled with conventional services' inability to meet Latinas' mental health needs are concerning given the exacerbated psychosocial sequelae experienced by Latinas with cancer.

The IOM reports the psychosocial impact of cancer overall is understudied, and highlights the disparity that exists among Latinos. The Latino cohort has become the largest minority population, which continues on a trajectory of substantial growth and a predicted exacerbation of cancer as the leading cause of death further intensifying the disparity that exists. It is imperative that culturally supported avenues of mental health support are utilized and encouraged among Latinos for their cancer psychosocial sequelae needs. This study brings to light key characteristics within the folk practice of Santería that can be harnessed by the field of social work to provide more culturally appropriate mental health services to our Latino clients with cancer. 


\section{REFERENCES}

Alferi, S. M., Antoni, M. H., Ironson, G., Kilbourn, K. M., \& Carver, C. S. (2001). Factors predicting the use of complementary therapies in a multi-ethnic sample of early stage breast cancer patients. Journal of American Medical Women's Association, 56(3), 120-123, 126.

Allan, G. A., \& Skinner, C. J. (1991). Handbook for research students in the social sciences. London: Falmer Press.

American Cancer Society. (2014). Cancer Facts and Figures 2014. Atlanta. Retrieved from http://www.cancer.org/acs/groups/content/@research/documents/ webcontent/acspc-042151.pdf

American Cancer Society. (2012a). Cancer Facts and Figures 2012. Atlanta. Retrieved from http://www.cancer.org/acs/groups/content/@epidemiologysurveilance/ documents/document/acspc-031941.pdf

American Cancer Society. (2012b). Cancer Facts \& Figures for Hispanics/Latinos 2012 -2014. Atlanta. Retrieved from http://www.cancer.org/acs/groups/content/@ epidemiologysurveilance/documents/document/acspc-034778.pdf

Anderson, L. M., Scrimshaw, S. C., Fullilove, M. T., Fielding, J. E., \& Normand, J. (2003). Culturally competent healthcare systems: a systematic review. American Journal of Preventive Medicine, 24(3S), 68-79.

Andrykowski, M. A., \& Kangas, M. (2010). Posttraumatic stress disorder associated with cancer diagnosis and treatment. In J. C. Holland et al. (Eds.), Psycho-Oncology (2nd ed.) (pp. 348-357). New York: Oxford University Press.

Annunziata, M. A., Muzzatti, B., \& Bidoli, E. (2011). Psychological distress and needs of cancer patients: A prospective comparison between the diagnostic and the therapeutic phase. Supportive Care in Cancer, 19(2), 291-295. doi: 10.1007/s00520-010-0818-9

Applewhite, S. L. (1995). Curanderismo: Demystifying the health beliefs and practices of elderly Mexican Americans. Health \& Social Work, 20(4), 247-253. doi: 10.1093/hsw/20.4.247

Armstrong, D., Gosling, A., Weinman, J., \& Marteau, T. (1997). The place of inter-rater reliability in qualitative research: An empirical study. Sociology, 31(3), 597-606. doi: 10.1177/0038038597031003015 
Ashing-Giwa, K. T. (2008). Enhancing physical well-being and overall quality of life among underserved Latina-American cervical cancer survivors: feasibility study. Journal of Cancer Survivorship, 2, 215-223. doi: 10.1007/s11764-0080061-2

Ashing-Giwa, K. T., Padilla, G. V., Bohorquez, D. E., Tejero, J. S., \& Garcia, M. (2006). Understanding the breast cancer experience of Latina women. Journal of Psychosocial Oncology, 24(3), 19-52. doi: 10.1300/J077v24n03_02

Ashing-Giwa, K. T., Padilla, G., Tejero, J., Kraemer, J., Wright, K., Coscarelli, A.,... Hills, D. (2004). Understanding the breast cancer experience of women: A qualitative study of African American, Asian American, Latina and Caucasian cancer survivors. Psychooncology, 13(6), 408-428. doi: 10.1002/pon.750

Baez, A., \& Hernandez, D. (2001). Complementary spiritual beliefs in the Latino community: The interface with psychotherapy. American Journal of Orthopsychiatry, 71(4), 408-415.

Balick, M. J., Kronenberg, F., Ososki, A. L., Reiff, M., Fugh-Berman, A., O’Connor, B., . . . Atha, D. (2000). Medicinal plants used by Latino healers for women's health conditions in New York City. Economic Botany, 54(3), 344-357. doi: 10.1007/BF02864786

Barbour, R. (2007). Doing focus groups. London: Sage.

Barnes, P. M., Bloom, B., \& Nahin, R. L. (2008). Complementary and alternative medicine use in adults and children: United States, 2007. National Health Statistics Report, 12, 1-23.

Belliard, J. C., \& Ramirez-Johnson, J. (2005). Medical pluralism in the life of a Mexican immigrant woman. Hispanic Journal of Behavioral Sciences, 27(3), 267285. doi: $10.1177 / 0739986305278130$

Berg, B. L. (2001). Qualitative research methods for the social sciences. Needham Heights, MA: Allyn \& Bacon.

Bernard, H. R. (2006). Research methods in Anthropology (4th ed.). Maryland: Altamira Press.

Bhui, K., Warfa, N., Edonya, P., McKenzie, K., \& Bhugra, D. (2007). Cultural competence in mental health care: a review of model evaluations. BMC Health Services Research, 7(15), 1-10. doi: 10.1186/1472-6963-7-15

Brandon, G. (1993). Santería from Africa to the New World: The dead sell memories. Bloomington, IN: Indiana University Press. 
Brown, D. H. (2003). Santería enthroned: Art, ritual, and innovation in an Afro-Cuban religion. Chicago: University of Chicago Press.

Buki, L. P., Garces, D. M., Hinestrosa, M. C., Kogan, L., Carrillo, I. Y., \& French, B. (2008). Latina breast cancer survivors' lived experiences: Diagnosis, treatment, and beyond. Cultural Diversity and Ethnic Minority Psychology, 14(2), 163-167. doi: 10.1037/1099-9809.14.2.163

Cabassa, L. J., Zayas, L. H., \& Hansen, M. C. (2006). Latino adults’ access to Mental Health Care: A review of epidemiological studies. Administration and Policy in Mental Health and Mental Health Services Research, 33(3), 316-330. doi: 10.1007/s10488-006-0040-8

Cabot, R. C. (1909). Social service and the art of healing. New York, NY: Moffat, Yard, \& Company.

Carpenter-Song, E., Whitley, R., Lawson, W., Quimby, E., \& Drake, R. E. (2011). Reducing disparities in Mental Health Care: Suggestions from the Dartmouth Howard Collaboration. Community Mental Health Journal, 47(1), 1-13. doi: 10.1007/s10597-009-9233-4

Chan, C.L.W., Ho, R.T.H., Lee, P.W.H., Cheng, J.Y.Y., Leung, P.P.Y., Foo, W., . . S Spiegel, D. (2006). A randomized controlled trial of psychosocial interventions using the psychophysiological framework for Chinese breast cancer patients. Journal of Psychosocial Oncology, (24)1, 3-26. doi: 10.1300/1077v24n01_02

Chang, H., Wallis, M., \& Tiralongo, E. (2007). Use of complementary and alternative medicine among people living with diabetes: Literature review. Journal of Advanced Nursing, 58(4), 307-319. doi: 10.1111/j.13652648.2007.04291.x

Changrani, J., Lieberman, M., Golant, M., Rios, P., Damman, J., \& Gany, F. (2008). Online cancer support groups: Experiences with underserved immigrant Latinas. Primary Psychiatry, 15(10), 55-62.

Cheung, F. K., \& Snowden, L. R. (1990). Community mental health and ethnic minority populations. Community Mental Health Journal, 26(3), 277-291. doi: $10.1007 / \mathrm{bf} 00752778$

Chin, J. L. (2000). Culturally competent health care. Public Health Reports, 115, 25-33.

Church of the Lukumi Babalu Aye. (2014, August 1). Membership. Retrieved from http://www.churchofthelukumi.com/clba-membership.html 
Clarke, K. M. (2008). Ritual change and the changing canon. In J. O. K. Olupona \& T. Rey (Eds.), Orisa devotion as world religion: The globalization of Yoruba religious culture (pp. 286-371). Madison, WI: University of Wisconsin Press.

Comas-Diaz, L. (2013). Comadres: The healing power of a female bond. Women \& Therapy, 36(1-2), 62-75. doi: 10.1080/02703149.2012.720213

Comas-Diaz, L. (2006). Latino healing: The integration of ethnic psychology into psychotherapy. Psychotherapy: Theory, Research, Practice, Training, 43(4), 436453. doi: 10.1037/0033-3204.43.4.436

Corbin, J., \& Strauss, A. (2008). Basics of qualitative research 3e: Techniques and procedures for developing grounded theory. Thousand Oaks, CA: Sage.

De La Cancela, V., \& Martinez, I. Z. (1983). An analysis of culturalism in Latino mental health: Folk Medicine as a case in point. Hispanic Journal of Behavioral Sciences, 5(3), 251-274. doi: 10.1177/07399863830053001

De La Rosa, M., Babino, R., Rosario, A., Martinez, N. V., \& Aijaz, L. (2012). Challenges and strategies in recruiting, interviewing, and retaining recent Latino immigrants in substance abuse and HIV epidemiologic studies. The American Journal on Addictions, 21(1), 11-22. doi: 10.1111/j.1521-0391.2011.00193.x

Delgado, M. (2007). Social Work with Latinos: A cultural assets paradigm. New York: Oxford University Press.

Delgado, M., \& Humm-Delgado, D. (1982). Natural support systems: Source of strength in Hispanic communities. Social Work, 27, 83-89.

Dodson, J. E., \& Batista, J. M. (2008). Sacred Spaces and Religious Traditions in Oriente Cuba. New Mexico: University of New Mexico Press.

Dye, J. F., Schatz, I. M., Rosenberg, B. A., \& Coleman, S. T. (2000). Constant comparison method: A kaleidoscope of data. The Qualitative Report, [On-line serial], 4(1/2). Available: http://www.nova.edu/ssss/QR/QR3-4/dye.html

Elder, J. P., Ayala, G. X., Parra-Medina, D., \& Talavera, G. A. (2009). Health communication in the Latino community: Issues and approaches. Annual Review of Public Health, 30, 227-251. doi: 10.1146/annurev.publhealth.031308.100300

Erwin, D. O., Trevino, M., Saad-Harfouche, F. G., Rodriguez, E. M., Gage, E., \& Jandorf, L. (2010). Contextualizing diversity and culture within cáncer control interventions for Latinas: Changing interventions, not cultures. Social Science \& Medicine, 71, 693-701. doi: 10.1016/j.socscimed.2010.05.005 
Fatone, A. M., Moadel, A. B., Foley, F. W., Fleming, M., \& Jandorf, L. (2007). Urban voices: The quality-of-life experience among women of color with breast cancer. Palliative \& Supportive Care, 5(02), 115-125. doi: 10.1017/S1478951507070186

Faugier, J., \& Sargeant, M. (1997). Sampling hard to reach populations. Journal of Advanced Nursing, 26, 790-797. doi: 10.1046/j.1365-2648.1997.00371.x

Fiske, A. P. (2002). Using individualism and collectivism to compare cultures - a critique of the validity of measurement of the constructs: Comment of Oyserman et al. (2002). Psychological Bulletin, 128(1), 78-88. doi: 10.1037//00332909.128.1.78

Fitzgerald, N. (2010). Acculturation, socioeconomic status, and health among Hispanics. NAPA Bulletin, 34(1), 28-46. doi: 10.1111/j.1556-4797.2010.01050.x

Florida Cancer Data System, University of Miami Miller School of Medicine (2014). Publications. Retrieved from http://fcds.med.miami.edu/inc/publications.shtml

Ford, K., Oberski, I., \& Higgins, S. (2000). Computer-aided qualitative analysis of interview data: Some recommendations for collaborative working. The Qualitative Report, 4(3-4). Retrieved from http://www.nova.edu/ssss/QR/QR43/oberski.html

Frisvold, N. D. M. (2011). Palo Mayombe: The garden of blood and bones. United Kingdom: Scarlet Imprint.

Gallant, M. P., Spitze, G., \& Grove, J. G. (2010). Chronic illness self-care and the family lives of older adults: A synthetic review across four ethnic groups. Journal of Cross Cultural Gerontology, 25, 21-43. doi: 10.1007/s10823-010-9112-z

Galvan, N., Buki, L. P., \& Garces, D. M. (2009). Suddenly, a carriage appears: Social support needs of Latina breast cancer survivors. Journal of Psychosocial Oncology, 27, 361-382. doi:10.1080/07347330902979283

Garcia, H., Sierra, A., \& Balam, G. (1999). Wind in the blood: Mayan healing and Chinese medicine. Berkeley, CA: North Atlantic Books.

Gloria, A. M., \& Peregoy, J. J. (1996). Counseling Latino alcohol and other substance users/abusers: Cultural considerations for counselors. Journal of Substance Abuse Treatment, 13(2), 119-126. doi: 0740-5472/96

Goncalves, V., Jayson, G., \& Tarrier, N. (2011). A longitudinal investigation of posttraumatic stress disorder in patients with ovarian cancer. Journal of Psychosomatic Research, 70(5), 422-431. doi: 10.1016/j.psychores.2010.09.017 
Gonzalez, M. J. (2002). Mental health intervention with Hispanic immigrants: Understanding the influence of the client's worldview, language, and religion. Journal of Immigrant \& Refugee Services, 1(1), 81-92. doi: 10.1300/J191v01n01_07

Gonzalez-Whippler, M. (2001). Santería: The Religion. St. Paul, MN: Llewellyn.

Graves, K.D. (2003). Social cognitive theory and cancer patients' quality of life: A meta-analysis of psychosocial intervention components. Health Psychology, 22(2), 210-219. doi: 10.1037/0278-6133.22.2.210

Guest, G., Bunce, A., \& Johnson, L. (2006). How many interviews are enough? An experiment with data saturation and variability. Field Methods, 18(1), 59-82. doi: $10.1177 / 1525822 X 05279903$

Gurung, R. A. R. (2013). A multicultural approach to health Psychology. American Journal of Lifestyle Medicine, 7(1), 4-12. doi: 10.1177/1559827612444548

Harwood, A. (1981). Introduction. In A. Harwood (Ed.), Ethnicity and medical care. Cambridge: Harvard University Press.

Hatfield, G. (2004). Encyclopedia of Folk Medicine: Old world and new world traditions. California: ABC-Clio.

Heathcote, J. D., West, J. H., Hall, P. C., \& Trinidad, D. R. (2011). Religiosity and utilization of Complementary and Alternative Medicine among foreign born Hispanics in the United States. Hispanic Journal of Behavioral Sciences, 33(3), 398-408. doi: 10.1177/0739986311410019

Helman, C. (1994). Culture, health, and illness: An introduction for health professionals ( $3^{\text {rd }}$ Ed.). Oxford: Butterworth Heinemann.

Hoffman, N. A. (2011). The requirements for culturally and linguistically appropriate services in health care. Journal of Nursing Law, 14(2), 49-57. doi: 10.1891/1073-7472.14.2.49

Holland, J. C., \& Weiss, T. R. (2010). History of Psycho-Oncology. In J. C. Holland, W. S. Breitbart, P. B. Jacobsen, M. S. Lederberg, M. J. Loscalzo, \& R. S. MsCorkle (Eds.), Psycho-Oncology $\left(2^{\text {nd }} e d\right)$ (pp. 3-14). New York, NY: Oxford.

Holliday, K. V. (2008). Religious healing and biomedicine in comparative context. In B. W. McNeil \& J. M. Cervantes (Eds.), Latina/o Healing Practices: Mestizo and Indigenous Perspectives (pp. 249-270). New York, NY: Routledge. 
Hopwood, P., Sumo, G., Mills, J., Haviland, J., \& Bliss, J. M. (2010). The course of anxiety and depression over 5 years of follow-up and risk factors in women with early breast cancer: Results from the UK Standardisation of Radiotherapy Trials (START). Breast, 19(2), 84-91. doi: 10.1016/j.breast.2009.11.007

Hsiao, A, Wong, M. D., Goldstein, M. S., Yu, H., Andersen, R. M., Brown, E. R., ... Wenger, N. S. (2006). Variation in Complementary and Alternative Medicine (CAM) use across racial/ethnic groups and the development of ethnic specific measures of CAM use. The Journal of Alternative and Complementary Medicine, 12(3), 281-290. doi: 10.1089/acm.2006.12.281

Hufford, D. J. (1997). Folk medicine and health culture in contemporary society. Primary Care: Clinics in Office Practice, 24(4), 723-741.

Humes, K., Jones, N. A., \& Ramirez, R. R. (2011). Overview of race and Hispanic origin: 2010. 2010 Census Briefs. Retrieved from http://www.census.gov/prod/cen2010/ briefs/c2010br-02.pdf

Hurdle, D. E. (2002). Native Hawaiian traditional healing: Culturally based interventions for social work practice. Social Work, 47(2), 183-192.

Insaf, T. Z., Jurkowski, J. M., \& Alomar, L. (2010). Sociocultural factors influencing delay in seeking routine health care among Latinas: A communitybased participatory research study. Ethnicity and Disease, 20, 148-154.

Institute of Medicine. (2002). Unequal treatment: Confronting racial and ethnic disparities in health care. Washington D.C.: National Academies Press.

Institute of Medicine. (2008). Cancer care for the whole patient: Meeting psychosocial health needs. Washington D.C.: The National Academies Press.

Jean-Pierre, P., Fiscella, K, Freund, K. M., Clark, J., Darnell, J., Holden, A.,... \& the Patient Navigation Research Program Group. (2010). Structural and reliability analysis of a patient satisfaction with cancer-related care measure: A multisite patient navigation research program study. Cancer, 117(4), 854-861. doi: 10.1002/cncr.25501

Jones, M. O., Polk, P. A., Flores-Peña, Y., \& Evanchuk, R. J. (2001). Invisible hospitals: Botánicas in ethnic health care. In E. Brady (Ed.), Healing Logics: Culture and Medicine in Modern Health Belief Systems (pp. 39-87). Logan, UT: Utah State University Press.

Justice-Gardiner, H., Nutt, S., Rechis, R., McMillan, B., \& Warf, R. (2012). Using media to reach Hispanic/Latino cancer survivors. Journal of Cancer Education, 27(1), 100-104. doi: 10.1007/s13187-011-0267-2 
Katon, W. J. (2003). Clinical and health services relationships between major depression, depressive symptoms, and general medical illness. Biological Psychiatry, 54(3), 216-226. doi: 10.1016/S0006-3223(03)00273-7

Kawaga-Singer, M. (1995). Socioeconomic and cultural influences on cancer care of women. Seminars in Oncology Nursing, 11(2), 109-119.

Keefe, S. E., \& Casas, J. M. (1980). Mexican-Americans and mental health: A selected review and recommendations for mental health service delivery. American Journal of Community Psychology, 8, 303-326.

Kleinman, A. (1980). Patients and healers in the context of culture. Berkeley: University of California Press.

Kleinman, A. (1988). The illness narratives. New York, N.Y.: Basic Books.

Koss, J. D. (1987). Expectations and outcomes for patients given mental health care or spiritist healing in Puerto Rico. American Journal of Psychiatry, 144(1), 56-61.

Laws, M. B., \& Carballeira, N. (2003). Use of nonallopathic healing methods by Latina women at midlife. American Journal of Public Health, 93(4), 525525.

Laycock, D. (1994). Free Exercise and the Religious Freedom Restoration Act. Fordham L. Review, 62, 883-892.

Livingston, G. (2009). Hispanics, health insurance and health care access. Pew Hispanic Center Reports, 1-5. Retrieved from http://www.pewhispanic.org/files/ reports/113.pdf

Lopez, S. R. (2002). Mental health care for Latinos: A research agenda to improve the accessibility and quality of mental health care for Latinos. Psychiatric Services, 53(12), 1569-1573.

Martinez, D., Aguado, C. X., Martinez, M., Flores, A. E., \& Meade, C. D. (2008). Development of a cancer camp for adult spanish-speaking survivors: Lessons learned from Camp Alegria. Journal of Cancer Education, 23(1), 4-9. doi: 10.1080/08858190701818234

McBride, D. F. (2011). Manifesting empowerment: How a family health program can address racism. Journal of Black Psychology, 37(3), 336-356. doi: 10.1177/0095798410390690

McCracken, G. (1988). The Long Interview. Qualitative Research Methods Series, 13. Newbury Park, CA: Sage. 
McKiernan, A., Steggles, S., Guerin, S., \& Carr, A. (2010). A controlled trial of group cognitive behavior therapy for Irish breast cancer patients. Journal of Psychosocial Oncology, 28, 143-156. doi: 10.1080/07347330903570511

McNeill, B. W., \& Cervantes, J. M. (2008). Counselors and curanderas/os: Parallels in the healing process. In B. W. McNeil \& J. M. Cervantes (Eds.), Latina/o healing practices: Mestizo and indigenous perspectives (pp. xvii-xxxiii). New York: Routledge.

McNeill, B. W., Esquivel, E., Carrasco, A., \& Mendoza, R. (2008). Santería and the healing process in Cuba and the United States. In B. W. McNeil \& J. M. Cervantes (Eds.), Latina/ o Healing Practices: Mestizo and Indigenous Perspectives (pp. 63-82). New York: Routledge.

Meade, C. D., Menard, J. M., Luque, J. S., Martinez-Tyson, D., \& Gwede, C. K. (2011). Creating community-academic partnerships for cancer disparities research and health promotion. Health Promotion Practice, 12(3), 456-462. doi:10.1177/1524839909341035

Miano, L. Y., Rojas, M. S., \& Trujillo, M. (1996). "Platicas y merienda”: Reaching Spanish-speaking patients in an oncology setting. Cancer Practice, 4(4), 199-203.

Moonesinghe, R., Zhu, J., \& Truman, B. I. (2011). Health insurance coverage United States, 2004 and 2008. Morbidity and Mortality Weekly Report, 60(01), 35-37.

Morgan, D. L. (1997). Focus groups as qualitative research. Thousand Oaks: Sage.

Moyer, A., Knapp-Oliver, S. K., Sohl, S. J., Schnieder, S., \& Floyd, A.H.L. (2009). Lessons to be learned from 25 years of research investigating psychosocial interventions for cancer patients. Cancer Journal, 15(5), 345-351. doi: 10.1097PPO.0b013e3181bf51fb

Murguia, A., Peterson, R. A., \& Zea, M. C. (2003). Use and implications of ethnomedical health care approaches among central American immigrants. Health and Social Work, 28(1), 43-51.

Murphy, J. M. (1994). Working the spirit. Boston, MA: Beacon Press.

Nairn, R. C., \& Merluzzi, T. V. (2003). The role of religious coping in adjustment to cancer. Psycho-oncology, 12(5), 428-441, doi: 10.1002/pon.654

Napoles-Springer, A. M., Ortiz, C., O’Brien, H., \& Diaz-Mendez, M. (2009). Develop in a culturally competent peer support intervention for Spanish-speaking Latinas with breast cancer. Journal of Immigrant and Minority Health, 11, 268-280. doi: 10.1007/ s10903-008-9128-4 
National Association of Social Workers. (2004). NASW Standards for Palliative and End of Life Care. https://www.socialworkers.org/practice/bereavement/standards/ standards0504New.pdf

Nunez, S. (2008). Brazil’s ultimate healing resource: The power of spirit. In B. W. McNeil \& J. M. Cervantes (Eds.), Latina/o healing practices: Mestizo and indigenous perspectives (pp. 139-174). New York, NY: Routledge.

O’Connor, M., Christensen, S., Jensen, A. B., Moller, S., \& Zachariae, R. (2011). How traumatic is breast cancer? Post-traumatic stress symptoms (PTSS) and risk factors for severe PTSS at 3 and 15 months after surgery in a nationwide cohort of Danish women treated for primary breast cancer. British Journal of Cancer, 104, 419-426. doi: 10.1038/sj.bjc.6606073

Office of Minority Health, U.S. Department of Health and Human Services. (2014). Cancer and Hispanic Americans. Retrieved from http://minorityhealth.hhs.gov/omh/browse.aspx?lvl=4\&lvlID=61

Ojelade, I. I., McCray, K., Ashby, J. S., \& Myers, J. (2011). Use of Ifá as a means of addressing mental health concerns among African American clients. Journal of Counseling and Development, 89, 406-412.

Olsen, S. A. (2009). A review of complementary and alternative medicine (CAM) by people with multiple sclerosis. Occupational Therapy International, 16(1), 57-70. doi: 10.1002/oti.266

Ortiz, F. A., \& Davis, K. G. (2008). Latina/o folk saints and Marian devotions: Popular religiosity and healing. . In B. W. McNeil \& J. M. Cervantes (Eds.), Latina/o healing practices: Mestizo and indigenous perspectives (pp. 29-62). New York: Routledge.

Ortiz, F. A., Davis, K. G., \& McNeill, B. W. (2008). Curanderismo: Religious and spiritual worldviews and indigenous healing traditions. In B. W. McNeil \& J. M. Cervantes (Eds.), Latina/o healing practices: Mestizo and indigenous perspectives (pp. 271-300). New York: Routledge.

Padgett, D. K. (2008). Qualitative methods in Social Work research (2 ${ }^{\text {nd }}$ ed.). Thousand Oaks, CA: Sage.

Padgett, D. K. (1998). Does the glove really fit? Qualitative research and clinical Social Work practice. Social Work, 43(4), 373-381. doi: 10.1093/sw/43.4.373

Pasquali, E. A. (1994). Santería. Journal of Holistic Nursing, 12(4), 380-390. doi: $10.1177 / 089801019401200407$ 
Patenaude, A. F., \& Kupst, M. J. (2005). Psychosocial functioning in pediatric cancer. Journal of Pediatric Psychology, 30(1), 9-27. doi: 10.1093/jpepsy/jsi012

Patton, M. Q. (2002). Two decades of developments in qualitative inquiry: A personal, experiential perspective. Qualitative Social Work, 1(3), 261-283. doi: $10.1177 / 1473325002001003636$

Paulino, A. (1996). Spiritism, Santeri, Brujeria, and Voodooism: A Comparative view of indigenous healing systems. Journal of Teaching in Social Work, 12(1-2), 105124. doi: 10.1300/J067v12n01_08

Perez, D., Ang, A., \& Vega, W. A. (2009). Effects of health insurance on perceived quality of care among Latinos in the United States. Journal of General Internal Medicine, 24(Suppl 3), 555-560. doi:10.1007/s11606-009-1080-z

Pescosolido, B. A., Gardner, C. B., \& Lubell, K. M. (1998). How people get into mental health services: Stories of choice, coercion and "muddling through" from first timers. Social Science \& Medicine, 46(2), 275-286.

Pew Hispanic Center. (2010). Hispanics and health care in the United States: Access, information and knowledge. Washington, D.C. Retrieved from http://pewhispanic.org/files/reports/91.pdf

Pew Hispanic Center (2007). Changing Faiths: Latinos and the Transformation of American Religion. Washington, D.C. Retrieved from http://www.pewhispanic.org/files/reports/75.pdf

Quinlan, M. B. (2011). Ethnomedicine. In M. Singer \& P. I. Erickson (Eds.), A Companion to Medical Anthropology (pp. 381-403). United Kingdom: WileyBlackwell.

Rahill, G. J. (2008). The practice and use of picuristes (lay injectionists) among Haitian immigrants in Miami-Dade County, Florida (Unpublished doctoral dissertation). Florida International University, Miami, FL.

Reiff, M., O’Connor, B., Kronenberg, F., Balick, M., \& Lohr, P. (2003). Ethnomedicine in the urban environment: Dominican healers in New York City. Human Organization, 62(1), 12-26.

Reyes-Ortiz, C. A., Rodriguez, M., \& Markides, K. S. (2009). The role of spirituality healing with perceptions of the medical encounter among Latinos. Journal of General Internal Medicine, 24(3 suppl), 542-547. doi: 10.1007/s11606-009-10679 
Ritter, L. A., \& Hoffman, N. A. (2011). Multicultural health. Sudbury, MA: Jones and Bartlett Publishers.

Ruiz, P., \& Langrod, J. (1976). The role of folk healers in Community Mental Health services. Community Mental Health Journal, 12(4), 392-398. doi: $10.1007 / \mathrm{bf01411078}$

Saha, S., Beach, M. C., \& Cooper, L. A. (2008). Patient centeredness, cultural competence and healthcare quality. Journal of National Medical Association, 100(11), 1275-1285.

Salami, A. (2008). Yoruba theology and tradition: The man and society. Nigeria: NIDD Limited.

Sanchez, M., Dillon, F., Ruffin, B., \& De La Rosa, M. (2012). The influence of religious coping on the acculturative stress of recent Latino immigrants. Journal of Ethnic \& Cultural Diversity in Social Work, 21(3), 171-194. doi: 10.1080/15313204.2012.700443

Sandoval, M. C. (1979). Santería as a mental health care system: An historical overview. Social Science and Medicine, 13(B), 137-151. doi: 0160-79/0601-0137S02.000

Sandoval, M. C. (2008). Santería in the Twenty-First Century. In J. K. Olupona \& T. Rey (Eds.), Orisa devotion as world religion: The globalization of Yoruba religious culture (pp. 355-371). Madison, WI: University of Wisconsin Press.

Santi, A. (1997). Santería compared to Psychology as a mental health care system. (Doctoral dissertation). Retrieved from Dissertation Abstracts International, 58, 1545B.

Santo, D. E. (2010). Spiritist boundary-work and the morality of materiality in AfroCuban religion. Journal of Material Culture, 15(1), 64-82. doi: $10.1177 / 1359183510355226$

Schim, S. M., \& Doorenbos, A. Z. (2010). A three-dimensional model of cultural congruence: Framework for intervention. Journal of Social Work End of Life Palliative Care, 6(3-4), 256-270. doi:10.1080/15524256.2010.529023

Schoenberg, N. E., Stoller, E. P., Kart, C. S., Perzynski, A., \& Chapleski, E. E. (2004). Complementary and alternative medicine use among a multiethnic sample of older adults with diabetes. Journal of Alternative and Complementary Medicine, 10(6), 1061-1066. doi: 10.1089/acm.2004.10.1061

Schwartz, D. (1985). Caribbean folk beliefs and western psychiatry. Journal of Psychosocial Nursing and Mental Health Services, 23, 26-30. 
Sellick, S. M., \& Edwardson, A. D. (2007). Screening new cancer patients for psychological distress using the hospital anxiety and depression scale. PsychoOncology 16, 534-542. doi: 10.1002/pon.1085

Silverman, D. (2000). Doing qualitative research: a practical handbook. London: Sage Publications.

Skinner, R. (1994). The Church of the Lukumi Babalu Aye, Inc. v. City of Hialeah: Still Sacrificing Free Exercising. Baylor L. Review, 46, 259-1147.

Smith, E. D. (1998). Preface. In E. R. Canda (Ed.), Spirituality in Social Work: New directions (pp. ix-x). Binghamton, NY: Hawthorne Press.

Smith, A. K., Sudore, R. L., \& Perez-Stable, E. J. (2009). Palliative care for Latino patients and their families: "Whenever we prayed, she wept". Journal of the American Medical Association, 301(10), 1047-E1. doi: 10.1001/jama.2009.308

Spencer, S. M., Lehman, J. M., Wynings, C., Arena, P., Carver, C. S., Antoni, M. H., . . Love, N. (1999). Concerns about breast cancer and relations to psychosocial well-being in a multiethnic sample of early-stage patients. Health Psychology, 18(2), 159-168. doi: 10.1037/0278-6133.18.2.159

Sperber, A. D. (2004). Translation and validation of study instruments for cross cultural research. Gastroenterology, 126(1), S124-S128. doi: 10.1053/j.gastro.2003.10.016

Spiegel, D., \& Giese-Davis, J. (2003). Depression and cancer: Mechanism and disease progression. Biological Psychiatry, 54(3), 269-282. doi: 10.1016/S00063223(03)00566-3

Stebbins, R. A. (2001). Exploratory research in the social sciences. Qualitative research methods series, 48. Thousand Oaks, CA: Sage.

Strauss, A., \& Corbin, J. (1998). Grounded theory methodology: An overview. In N. K. Denzin \& Y. S. Lincoln (Eds.), Handbook of qualitative research (pp. 273285). Thousand Oaks, CA: Sage.

Suarez, M., Raffaelli, M., \& O’Leary, A. (1996). Use of folk healing practices by HIV- infected Hispanics living in the United States. AIDS Care, 8(6), 683-690. doi: 10.1080/09540129650125399

Tafur, M. M., Crowe, T. K., \& Torres, E. (2009). A review of curanderismo and healing practices among Mexicans and Mexican Americans. Occupational Therapy International, 16(1), 82-88. doi: 10.1002/oti.265 
Taylor, S., \& Bogden, R. (1998). Introduction to qualitative research methods ( $3^{\text {rd }}$ ed.). New York: Wiley.

Trochim, W., \& Donnelly, J. P. (2007). The research methods knowledge base ( $3^{\text {rd }}$ ed.). Mason, OH: Atomic Dog Publishing.

U.S. Census Bureau. (2012). American Fact Finder, Selected Economic Characteristics, 2006-2012 American Community Survey Selected Population Tables. Retrieved from: http://factfinder2.census.gov/faces/tableservices/jsf/pages/productview.x html?pid=ACS_11_1YR_CP03\&prodType=table

Viladrich, A. (2006). Botanicas in America's backyard: Uncovering the world of Latino healers' herb-healing practices in New York City. Human Organization, 65(4), 407-419. doi: 0018-7259/06/040407-1351.80/1

Viladrich, A., \& Abraido-Lanza, A. F. (2009). Religion and mental health among minorities and immigrants in the U.S. In S. Loue \& M. Sajatovic (Eds.), Determinants of minority health and wellness (pp. 149-174). doi: 10.1007/978-0387-75659-2_8

Villa-Caballero, L., Morello, C. M., Chynoweth, M. E., Prieto-Rosinol, A., Polonsky, W. H., Palinkas, L. A., \& Edelman, S. V. (2010). Ethnic differences in complementary and alternative medicine use among patients with diabetes. Complementary Therapies in Medicine, 18(6), 241-248. doi: 10.1016/j.ctim.2010.09.007

Wolff, B., Knodel, J., \& Sittitrai, W. (1993). Focus groups and surveys as complementary research methods: A case example. In D. L. Morgan (Ed.), Successful focus groups: Advancing the state of the art (pp. 118-136). Thousand Oaks: Sage.

Wuaku, A. (2011, October 10). [Class lecture]. Santería. Florida International University, Miami, FL.

Yanez, B., Thompson, E. H., \& Stanton, A. L. (2011). Quality of life among Latina breast cancer patients: A systematic review of the literature. Journal of Cancer Survivorship, 5, 191-207. doi: 10.1007/s11764-011-0171-0

Zambrana, R. E., Molnar, C., Munoz, H. B., \& Lopez, D. S. (2004). Cultural competency as it intersects with racial/ethnic, linguistic, and class disparities in managed healthcare organizations. The American Journal of Managed Care, 10(SP), SP37SP44.

Zapata, J., \& Shippee-Rice, R. (1999). The use of folk healing and healers by six Latinos living in New England: A preliminary study. Journal of Transcultural Nursing, 10(2), 136-142. doi: $10.1177 / 10436596$ 
APPENDICES 
Appendix A

Stage One Interview guide (ENG)

1. Please tell me the thoughts and feelings the diagnosis provokes? Psychologically, emotionally, what are you experiencing? How are you feeling about your life, your obligations, your social roles, your job, your family and kids, etc.?

2. Why did you decide to consult with a healer in regards to your illness?

3. I'm interested in your experience with Santería. Please talk to me about the things that go on while you're there.

a. Probes: What does the healer do? What you do? What's going on around you? Are there others with you?

4. Please tell me about the thoughts and feelings you experience while you are engaged in a session with the Santería healer.

5. And please tell me about the thoughts and feelings you experience after being there regarding your illness. How did that consultation impact your thoughts, feelings, what you were experiencing psychologically and emotionally about your illness?

6. What was different about this than going to see your doctors, a therapist, a support group with other women living with the experience of cancer, etc.?

7. Please describe what is most meaningful about receiving these services from the Santería healer. What gives the doctors the ability to heal you?

8. What gives the medical doctors the ability to heal you?

9. What gives the Santería healer his/her ability to heal you?

10 . What is your religious affiliation?

11. How does the spiritual healing the Santero/s performs differ from seeking help from a priest, a minister, a church group, or prayer?

12. What do you know about the spirits and/or the orisha that came to your aid? OR What do you know about the spirits and/or the orisha that the santero/a said came to your aid?

What gives the spirits and/or the orisha the authority to heal you?

OR What are their qualities that heal you?

13. Can you describe to me what the experience of going to the oncology doctor is like for you?

14. Can you describe what going to a psychologist or a therapist is like for you?

a. If no...Can you describe what you think going to a psychologist or a therapist would be like?

15. Can you describe what going to a support group for women with cancer is like for you?

a. If no...Can you describe what you think going to a support group would be like?

16. Who were the people you were able to go to where you could be completely honest and transparent about your reactions, thoughts and feelings about your cancer?

17. Did you tell any of your Dr.'s that you also consulted a folk healer?

a. If no... why not? 
b. What did you think their reaction would be?

c. What do you think they're opinion of you would be regarding your decision see a folk healer?

18. You mentioned that your religious affiliation is -------. How do you negotiate ascribing to that religion and its doctrine, and practicing Santería?

a. How does it clash? How does it not?

19. Does your family use Santería services? Do they know that you've used it? What is your family's opinion on Santería?

Prompts to be used throughout to encourage elaboration:

Tell me more about that. Can you describe that more? Can you add anything to what you said about ----

\section{DEMOGRAPHIC CHARACTERISTICS}

Age:

Country of birth:

(If native born) Where in the U.S.:

(If foreign born) When did you emigrate to the U.S.:

How long living in Miami-Dade County:

Hispanic subgroup you identify with:

Highest grade of education completed:

Occupation:

Marital Status:

Members of household:

How many children:

(If 1 or more) Ages:

Are they dependent on you:

Yearly household income:

Any insurance coverage (private, Medicaid, JMH): Husband's

\section{ONCOLOGY}

Cancer site:

Date diagnosed:

Stage diagnosis:

Is current cancer diagnosis initial or re-current:

(If recurrent) Initially diagnosis date:

Initial diagnosis site:

Initial diagnosis stage:

Oncology treatments received for initial diagnosis:

Date treatment began:

Length of treatment:

Time in remission:

Current oncological treatments receiving:

Date began treatment:

Length of treatment (or anticipated length): 


\section{Appendix B \\ Stage One Interview Guide (SPN)}

1. Por favor dígame los pensamientos y sentimientos que el diagnostic provoca. Psicologicamente, emocionalmente, con que estas tratando? Cómo se siente acerca de su vida, sus obligaiones, sus roles sociales, su trabajo, su familia, y los niños, etc.?

2. ¿Por qué decidió consultar a un curandero en respecto a su endermedad?

3. Estoy interesado en su experiencia con la Santería. Por favor hablar conmigo sobre las cosas que suceden mientras estás allí.

Probes: ¿Que hace el curandero? ¿Qué hace usted durante? Qué está pasando alrededor de tí? ¿Hay otras personas con usted?

4. Por favor dígame acerca de los pensamientos y sentimientos que suceden mientras que ested está comprometido en una session con el curandero de Santería.

5. Por favor dígame acerca de los pensamientos y sentimientos que suceden con respecto a su enfermedad despues de estar allí con el curandero de Santería.

6. ¿Qué es diferente acerca de esto en lugar de ir a ver a sus medicos, un terapista, un grupo de apoyo con otras mujeres que viven con la experienca del cancer?

7. Por favor describa lo que es más significativo sobre la recepción de estos servicios del curandero de Santería.

8. Que es lo que le da a los doctores la habilidad para curarle?

9. Que es lo que da al curandero de Santería la habilidad para curarle.

10. Cuál es tu afiliación religiosa?

11. Como es la diferencia de la curación spiritual que el/los Santero(s) hacen distinto a la ayuda de un cura, pastor, grupo de iglesia o grupo de oración?

12. Que es lo que sabe de espíritus y/o las orishas que viene a su ayuda? O que sabe de los espíritus y/o la orisha que el santero (a) dijo que iba venir a su ayuda?

13. Que es lo que da a los espíritus y /o a la orisha la autoridad de curarle?

14. Me puede describir como la experiencia de ir a un oncólogo es para ti?

15. Me puede describir como yendo a un sicólogo o terapeuta es para ti?

a. Si no... Me puede describir como usted piensa yendo a un sicólogo o terapeuta podría ser?

16. Me puede describir como yendo a un grupo de apoyo para mujeres con cáncer fue para usted?

a. Si no.... Me puede describir como usted piensa yendo a un grupo de apoyo podría ser?

17. Quien fueron las personas que usted pudo ir a donde usted pudo ser completamente honesta y transparente sobre sus reacciones, pensamientos y sentimientos sobre su cáncer?

18. Les conto a cualquiera de sus doctores que también había consultado con un curandero?

a. Si no... porque no?

b. Qué piensa usted que la reacción del doctor seria?

c. Que es lo que piensa que la reacción del doctor sobre usted seria sobre su decisión de ver a un curandero? 
19. Me menciones que su afiliación religiosa es .... Como usted negocia asignándote a esa religión y su doctrina y practicando la Santería?

a. Como esta esto en desacuerdo? Como no está en desacuerdo?

20. Su familia usa los servicios de la santería? Saben que usted lo ha utilizado?

Cuales son las opiniones de sus familiares sobre la santería?

Solicita que se utilizerá para formentar elaboración:

Cuéntame mas sobre eso. ¿Puede describer eso más?

¿Se puede añadir algo a lo que has dicho sobre ----?

\section{Características Demográficas}

Edad:

País de nacimiento:

(Si eres nacida natal) Donde en los E.E. U.U?

(Si eres nacida en el extranjero) Cuando emigraste a los E.E. U.U?

Cuanto tiempo ha estado viviendo en el condado de Miami-Dade?

Grupo de Hispano con que se identifica:

Nivel más alto de educación que ha completado:

Empleo:

Estado Civil:

Miembros de su hogar:

Cuantos niños/niñas:

(Si 1 o más) Edades:

Son dependientes de usted?:

Salario Anual de su hogar:

Alguna cubertura de seguros (privado, Medicaid, JMH):

Oncología

Donde está localizado el cancer?:

La fecha que ha sido diagnosticada:

La etapa de cáncer diagnosticada:

Es el diagnostico de su actual cáncer inicial o recurrente?:

(Si es recurrente) La fecha inicial de su diagnostic?:

Donde está localizado el diagnóstico inicial?:

En que etapa fue diagnóstica inicialmente?:

Tratamiento oncológico recibido después del primer diagnostic?:

La fecha inicial de su tratamiento:

El tiempo transcurrido de tratamiento:

El tiempo en remisión:

Actual tratamientos oncológicos que está recibiendo:

La fecha que comenzó su tratamiento:

Duración de tratamiento (o anticipado duración):

El tiempo de tratamiento (o el tiempo anticipado de su tratamiento): 
VITA

ADELAIDA M. ROSARIO

Born, Agana Heights, Guam

2004

B.A., Psychology

Florida International University

1999-2004

Research Assistant

University of Miami, Miller School of Medicine

2005-2007

Social Worker II

Guam Public School System

2009

M.A. Micronesian Studies

University of Guam

2010-2013

Instructor on Record, Social Work

Florida International University

2014-Present

Health Specialist

National Institutes of Health, NIMHD

\section{PUBLICATIONS AND PRESENTATIONS}

Rosario, A. (2010). Meeting Chamorro women's primary health care needs: Examining the cultural impact of mamahlao on gynecological screening. Pacific Health Dialog, 16(1), 81-90.

Rosario, A. (2010). Recruiting, Interviewing, and Retaining Recent Latino Immigrants in Longitudinal Studies: Lessons Learned and Recommendations. Poster presented at Latino Social Workers’ Organization Annual Conference, Chicago, Illinois.

Rosario-Hoyos, A. (2011). Meeting Chamorro Women's Primary Health Care Needs. Poster presented at the Society for Social Work \& Research Annual Conference, Tampa, Florida.

De La Rosa, M., Babino, R., Rosario, A., Martinez, N. V., \& Aijaz, L. (2012). Challenges and strategies in recruiting, interviewing, and retaining recent Latino immigrants in substance abuse and HIV epidemiologic studies. The American Journal on Addictions, 21(1), 11-22. 
Rosario, A. M. (2012). Folk and Traditonal Healer Use Among Latinos with Chronic Illnesses: Psychosocial Sequelae Support. Poster presented at the American Public Health Association Annual Meeting, San Francisco, California.

Rosario, A. M., \& De La Rosa, M. (2014). Santería as Informal Mental Health Support Among U.S. Latinos with Cancer. Journal of Religion \& Spirituality in Social Work: Social Thought, 33(1), 4-18. 